\title{
Complex fermatean fuzzy $N$-soft sets: a new hybrid model with applications
}

\author{
Muhammad Akram ${ }^{1}$ Umaira Amjad ${ }^{1} \cdot$ José Carlos R. Alcantud ${ }^{2} \cdot$ Gustavo Santos-García $^{3}$ (D)
}

Received: 21 March 2021 / Accepted: 30 November 2021

(C) The Author(s) 2022

\begin{abstract}
Decision-making methods play an important role in the real-life of human beings and consist of choosing the best options from a set of possible choices. This paper proposes the notion of complex Fermatean fuzzy $N$-soft set $\left(\mathrm{CFFNS}_{f} \mathrm{~S}\right)$ which, by means of ranking parameters, is capable of handling two-dimensional information related to the degree of satisfaction and dissatisfaction implicit in the nature of human decisions. We define the fundamental set-theoretic operations of $\mathrm{CFFNS}_{f} \mathrm{~S}$ and elaborate the $\mathrm{CFFS}_{f} \mathrm{~S}$ associated with threshold. The algebraic and Yager operations on $\mathrm{CFFNS}_{f}$ numbers are also defined. Several algorithms are proposed to demonstrate the applicability of $\mathrm{CFFNS}_{f} \mathrm{~S}$ to multi-attribute decision making. The advanced algorithms are described and accomplished by several numerical examples. Then, a comparative study manifests the validity, feasibility, and reliability of the proposed model. This method is compared with the Fermatean fuzzy Yager weighted geometric $\left(\mathrm{FFY}_{w} \mathrm{G}\right)$ and the Fermatean fuzzy Yager weighted average $\left(\mathrm{FFY}_{w} \mathrm{~A}\right)$ operators. Further, we developed a remarkable CFFNS $_{f}$-TOPSIS approach by applying innovative CFFNS $_{f}$ weighted average operator and distance measure. The presented technique is fantastically designed for the classification of the most favorable alternative by examining the closeness of all available choices from particular ideal solutions. Afterward, we demonstrate the amenability of the initiated approach by analyzing its tremendous potential to select the best city in the USA for farming. An integrated comparative analysis with existing Fermatean fuzzy TOPSIS technique is rendered to certify the terrific capability of the established approach. Further, we decisively investigate the rationality and reliability of the presented $\mathrm{CFFNS}_{f} \mathrm{~S}$ and $\mathrm{CFFNS}_{f}$-TOPSIS approach by highlighting its advantages over the existent models and TOPSIS approaches. Finally, we holistically describe the conclusion of the whole work.
\end{abstract}

Keywords Complex Fermatean fuzzy set $\cdot N$-soft set $\cdot$ Decision making

\section{Introduction}

Multi-attribute decision-making (MADM) methods play an important role in the real life of human beings. The process of choosing the best option among a set of possible options is present in all human activities. Decision making in the domain of crisp sets to handle exact and precise data has been a growing field of research for mathematicians.

J.C.R. Alcantud is grateful to the Junta de Castilla y León and the European Regional Development Fund (Grant CLU2019-03) for the financial support to the Research Unit of Excellence "Economic Management for Sustainability" (GECOS). The work of Santos-García was partially supported by the Spanish project TRACES TIN2015-67522C3-3-R.

Extended author information available on the last page of the article

\subsection{Related work}

Given the dubious and erroneous nature of human decisions, the limitations of decision making in the area of crisp set have gained importance over time. Zadeh (1965) was the pioneer who coped with the fuzziness and ambiguity of human decisions in the field of decision making. Fuzzy set (FS) theory refined not only the decision making, but also the related fields like social sciences, production management, etc. (Abdullah et al. 2012; Alcantud and Andrés Calle 2017; Guiffrida and Nagi 1998). FS theory empowers the experts to use their complacency level (membership/belongingness degree) with attributes whose values are between 0 and 1.

Undoubtedly, FS theory allows to work with unsettling analysis in the field of decision making. Nevertheless, FS theory could not assess the nature of satisfaction and 
dissatisfaction with human decisions. To salvage these shortcomings, Atanassov (1986) extended the FS with intuitionistic fuzzy sets (IFS) and added the non-membership function which is limited to the interval $[0,1]$ in order to express the level of discontent with human decisions. In his model the sum of satisfaction and dissatisfaction degrees is in the unit interval.

In 2013, Yager (2013a, 2013b) adapted the conditions of IFS, to present the novel concept of Pythagorean fuzzy set (PFS) with relaxing conditions that the sum of square of belongingness degree and non-membership degree should enclose in unit interval. Due to the constraints in PFS, Yager (2016) introduced the model of q-rung orthopair fuzzy set (q-ROFS) with conditions that sum of $\mathrm{q}^{\text {th }}$ power of belongingness degree and non-membership degree should not exceed from 1. Later on, Senapati and Yager (2020) developed the theory of Fermatean fuzzy set (FFS) that is more general model than IFS and PFS in which the cubic sum of membership degree and non-membership degree should lie in unit interval. FFS as an extension of IFS and PFS can support more amount of inexactness and vagueness that provide more precise results in decision making framework.

Aforementioned models were not applicable in 2-dimensional problems. Thus, Ramot et al. (2002) introduced the complex fuzzy set (CFS) which was proposed by the emerging relationship of complex and FS theory having complex unit circle as the range of membership function that enables the CFS to handle the 2-dimensional information along with amplitude and phase terms. The amplitude part and phase part both are real-valued functions which can take values from the unit interval to show the vagueness of both dimensions. Later, Alkouri and Salleh (2012) put forward the idea of complex intuitionistic fuzzy set (CIFS), in order to describe the non-membership degree along with membership degree within the complex unit circle, where the sum of phase terms and amplitude terms of belongingness degree and falseness degree should not exceed from 1. Further, Akram and Naz (2019) \& Ullah et al. (2020) presented the new model of complex Pythagorean fuzzy set (CPFS), as an extension of CIFS, which has more generalized structure than CFS and CIFS as it possesses more relaxed conditions on the phase and amplitude terms.

The idea of soft sets $\left(\mathrm{S}_{f} \mathrm{Ss}\right)$ theory was proposed by Molodtsov (1999), who also presented its relevancy and remarkable significance in the fields of operational research, probability theory, game theory and smoothness of functions (Molodtsov 1999, 2004). Alcantud and Santos-García (2017) proposed a totally revised approach for $\mathrm{S}_{f} \mathrm{~S}$ based decision-making issues under imperfect information. Many researchers brought up many models to enhance the literature of $\mathrm{S}_{f} \mathrm{~S}$, inclusive of fuzzy $\mathrm{S}_{f} \mathrm{Ss}$ $\left(\mathrm{FS}_{f} \mathrm{Ss}\right.$ ) (Maji et al. 2001b), Intuitionistic $\mathrm{FS}_{f} \mathrm{Ss}\left(\mathrm{IFS}_{f} \mathrm{Ss}\right)$ (Maji et al. 2001a), Pythagorean $\mathrm{FS}_{f} \mathrm{Ss}\left(\mathrm{PFS}_{f} \mathrm{Ss}\right.$ ) (Peng et al. 2015), Fermatean $\mathrm{FS}_{f}$ Ss $\left(\mathrm{FFS}_{f} \mathrm{Ss}\right.$ ) (Sivadas and John 2020), et cetera. The idea of a new perspective for the selection of best alternatives problems based on $\mathrm{FS}_{f} \mathrm{Ss}$ was given by Alcantud (2016). Fatimah et al. (2019) worked on a new structure of $\mathrm{S}_{f} \mathrm{Ss}$, namely, probabilistic $\mathrm{S}_{f} \mathrm{~S}$. Alcantud et al. (2017) proposed a new hybrid model named as valuation fuzzy $\mathrm{S}_{f} \mathrm{~S}$ and used it for real case study that uses data from the Spanish real estate market.

From latest studies of hybrid $\mathrm{S}_{f} \mathrm{~S}$ models, it can be concluded that primarily work of the researchers was based on real numbers between $[0,1]$ or binary evaluation in $\mathrm{S}_{f} \mathrm{~S}$ models (Ma et al. 2017). But nowadays, objects are evaluated by non-binary structures such as voting system and rating or ranking objects. Due to that, numerous researchers for instance Alcantud and Laruelle (2014), Chen et al. (2013), and Herawan and Deris (2009) have worked in formal models for non-binary evaluations. Stimulated by these concerns, Fatimah et al. (2018) proposed the model of $N$-soft set $\left(\mathrm{NS}_{f} \mathrm{~S}\right)$ which is an extension of $\mathrm{S}_{f} \mathrm{~S}$ and encapsulate the idea of parameterized characterization of the alternatives that depend on the finite number of ordered grades. Fatimah and Alcantud (2021) introduced the idea of multi-fuzzy $\mathrm{NS}_{f} \mathrm{~S}$. Later on, Akram et al. (2018, 2021b, 2021d) combined the concept of $\mathrm{NS}_{f} \mathrm{~S}$ with FS and explored the new hybrid model, namely, fuzzy $\mathrm{N}$ soft set $\left(\mathrm{FNS}_{f} \mathrm{~S}\right)$. This novel concept involves the finite number of ordered grades along with the vagueness in the conception of the attributes that are used for decision making. Another hybrid model called the hesitant $N$-soft set was introduced by Akram et al. (2019a). Akram et al. (2019b) extended the idea of $\mathrm{FNS}_{f} \mathrm{~S}$ and presented the hybrid model of intuitionistic fuzzy $N$-soft set $\left(\mathrm{IFNS}_{f} \mathrm{~S}\right.$ ) that can also capture the non-membership grades. Moreover, Zhang et al. (1965) extended $\mathrm{IFNS}_{f} \mathrm{~S}$ to Pythagorean fuzzy $N$-soft set $\left(\mathrm{PFNS}_{f} \mathrm{~S}\right)$ that possesses more relaxed conditions than existing models. Recently, Akram and his contributors set forth the hybrid models of bipolar $\mathrm{FNS}_{f}$ Ss (Akram et al. 2021a), complex spherical $\mathrm{FNS}_{f}$ Ss (Akram et al. 2021c) and complex Pythagorean $\mathrm{FNS}_{f}$ Ss $\left(\mathrm{CPFNS}_{f} \mathrm{Ss}\right)$ (Akram et al. 2021e).

The characteristic comparison of proposed and existing models is organized in Table 1 that present a broad view concerning the superiority of the manifested model.

In recent years, a technique for order preference by similarity to the ideal solution (TOPSIS) was proposed by Hwang and Yoon (1981) to solve the MADM problems. The basic idea of TOPSIS technique is to find out the best opt which is closest to the positive ideal solution (PIS) and farthest away from the negative ideal solution (NIS). Chen 
Table 1 Characteristic comparison of the proposed and existent models

\begin{tabular}{|c|c|c|c|c|}
\hline Models & $\begin{array}{l}\text { Capable to address } \\
\text { imprecise information }\end{array}$ & $\begin{array}{l}\text { Capable to address 2-D } \\
\text { information }\end{array}$ & $\begin{array}{l}\text { Capable to address rating-based } \\
\text { parameterized information }\end{array}$ & $\begin{array}{l}\text { Have the features of } \\
\text { generalization }\end{array}$ \\
\hline FS (Zadeh 1965) & レ & $\times$ & $\times$ & $\times$ \\
\hline $\begin{array}{l}\mathrm{NS}_{f} \mathrm{~S} \text { (Fatimah } \\
\text { et al. 2018) }\end{array}$ & $x$ & $\times$ & $\nu$ & $\times$ \\
\hline $\begin{array}{l}\mathrm{FNS}_{f} \mathrm{~S}(\mathrm{Akram} \\
\text { et al. 2018) }\end{array}$ & レ & $x$ & & レ \\
\hline $\begin{array}{l}\mathrm{IFNS}_{f} \mathrm{~S}(\mathrm{Akram} \\
\text { et al. 2019b) }\end{array}$ & レ & $x$ & レ & レ \\
\hline $\begin{array}{l}\mathrm{PFNS}_{f} \mathrm{~S} \text { (Zhang } \\
\text { et al. 1965) }\end{array}$ & レ & $x$ & レ & $\nu$ \\
\hline $\begin{array}{l}\mathrm{CPNS}_{f} \mathrm{~S}(\mathrm{Akram} \\
\text { et al. 2021e) }\end{array}$ & レ & $\boldsymbol{}$ & レ & 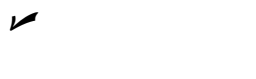 \\
\hline $\begin{array}{l}\mathrm{CFFNS}_{f} \mathrm{~S} \\
\quad \text { (proposed) }\end{array}$ & レ & レ & レ & 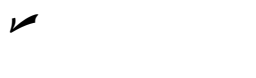 \\
\hline
\end{tabular}

(2000) utilized the TOPSIS technique for multi-attribute group decision-making (MAGDM) under a fuzzy environment. Li et al. (2019) applied the fuzzy TOPSIS approach for the case-study of the Beijing rail transportation system. Boran et al. (2009, 2011, 2012) built up the theory of intuitionistic fuzzy TOPSIS (IF-TOPSIS) and presented various real applications related to technology and business. Akram and his collaborators proposed the methodologies of interval-valued hesitant fuzzy TOPSIS (Akram and Adeel 2019), Pythagorean Fuzzy TOPSIS (PF-TOPSIS) (Akram et al. 2019c), and complex Pythagorean fuzzy TOPSIS (CPF-TOPSIS) (Akram et al. 2020) to address the tricky MAGDM problems. Senapati and Yager (2020) put forward the Fermatean fuzzy TOPSIS (FF-TOPSIS) to capture the MADM problems. Eraslan (2015) redesigned the TOPSIS approach under the environment of $\mathrm{S}_{f} \mathrm{~S}$ ( $\mathrm{S}_{f}$-TOPSIS) and illustrated the methodology by means of its potential application. Eraslan and Karaaslan (2015) adapted the approach of TOPSIS under the framework of $\mathrm{FS}_{f} \mathrm{Ss}$ ( $\mathrm{FS}_{f}$-TOPSIS) and demonstrated its cogent applications to select the suitable house. Han et al. (2019) extended the technique of TOPSIS under entropy on $\mathrm{PFS}_{f}$ Ss environment and implemented it for the selection of missile position. Salsabeela and John presented

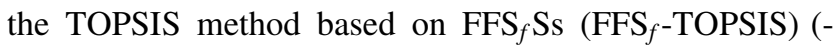
Salsabeela and John 2021) and elaborated it with the practical application for the selection of supplier for fivestar hotel.

The comparison of proposed and existing techniques based on TOPSIS method, according to their characteristics, is arranged in Table 2 which provide an extensive view about the dominance of the presented methodology.

\subsection{Motivation}

The motivation of the proposed hybrid model is given by the following facts:

- The idea of $\mathrm{NS}_{f} \mathrm{~S}$ captures the graded parameterized information but it has no potential to handle the fuzziness and vagueness of the provided data.

- The brilliant models of CIFS and CPFS are competitive frameworks for capturing the 2-dimensional vague data simultaneously. But they also have some restrictions due to the inadequacy of ranking based criteria.

- Moreover, the $\mathrm{FFS}_{f} \mathrm{~S}$ theory outstandingly renders the binary parameterized mechanism that handles ambiguity and vagueness of information with fantastic universality. But still, it is a 1-dimensional model that cannot present the uncertain periodic information as well as unable to cope with the ordered graded parameters of tricky practical problems.

- The decision-making technique based models $\mathrm{FNS}_{f} \mathrm{~S}$, $\mathrm{IFNS}_{f} \mathrm{~S}$ and $\mathrm{PFNS}_{f} \mathrm{~S}$ can only deal with 1-dimensional data. None of the described models can handle 2-dimensional problems.

- Further, the novel idea of $\mathrm{CPFNS}_{f} \mathrm{~S}$ is an efficacious model with splendid characteristics to handle the obscurity of parameterized fuzzy information. Despite that, it has some flaws that spring up due to its restricted space.

- Classical TOPSIS technique is specifically devised to determine the optimal solution based on the assessed closeness of the preferences choices from the ideal solution. But this hypothetical technique must be altered to tackle the ordered graded obscurity and vagueness of inexact information. 
Table 2 Characteristic comparison of proposed and existing TOPSIS techniques

\begin{tabular}{|c|c|c|c|c|}
\hline Approaches & $\begin{array}{l}\text { Have capability to deal } \\
\text { uncertain information }\end{array}$ & $\begin{array}{l}\text { Have capability to } \\
\text { deal 2-D information }\end{array}$ & $\begin{array}{l}\text { Have capability to deal } \\
\text { parameterized information }\end{array}$ & $\begin{array}{l}\text { Have capability to deal rating- } \\
\text { based parameterized information }\end{array}$ \\
\hline $\begin{array}{l}\text { Fuzzy-TOPSIS } \\
\text { technique (Chen 2000) }\end{array}$ & 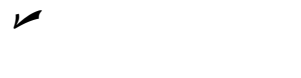 & $\times$ & $\times$ & $x$ \\
\hline $\begin{array}{l}\text { IF-TOPSIS } \\
\text { technique (Boran et al. } \\
\text { 2009) }\end{array}$ & 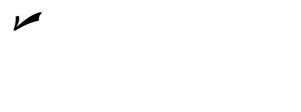 & $\times$ & $\times$ & $\times$ \\
\hline $\begin{array}{l}\text { PF-TOPSIS } \\
\text { technique (Akram et al. } \\
\text { 2019c) }\end{array}$ & 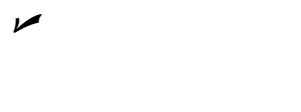 & $\times$ & $\times$ & $x$ \\
\hline $\begin{array}{l}\text { FF-TOPSIS } \\
\text { technique (Senapati and } \\
\text { Yager 2020) }\end{array}$ & レ & $\times$ & $\times$ & $\times$ \\
\hline $\begin{array}{l}\text { CPF-TOPSIS } \\
\text { technique (Akram et al. } \\
2020 \text { ) }\end{array}$ & レ & レ & $\times$ & $\times$ \\
\hline $\begin{array}{l}\mathrm{S}_{f} \text {-TOPSIS } \\
\quad \text { technique (Eraslan 2015) }\end{array}$ & $\times$ & $\times$ & V & $\times$ \\
\hline $\begin{array}{l}\mathrm{FS}_{f} \text {-TOPSIS } \\
\text { technique (Eraslan and } \\
\text { Karaaslan 2015) }\end{array}$ & レ & $\times$ & V & $\times$ \\
\hline $\begin{array}{l}\text { FFS }_{f} \text {-TOPSIS } \\
\text { technique (Salsabeela and } \\
\text { John 2021) }\end{array}$ & 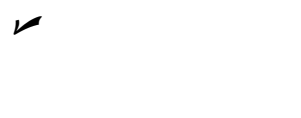 & $\times$ & レ & $\times$ \\
\hline $\begin{array}{l}\mathrm{CFFNS}_{f} \text {-TOPSIS technique } \\
\text { (proposed) }\end{array}$ & レ & レ & $\nu$ & \\
\hline
\end{tabular}

Because of all these constraints motivated us to put forward the idea of a ground-breaking hybrid model called $\mathrm{CFFNS}_{f}$ Ss along with $\mathrm{CFFNS}_{f}$-TOPSIS approach which competently handles two-dimensional information with relaxed conditions that cubic sum of amplitude and phase terms belongs to the interval $[0,1]$. Moreover, $\mathrm{CFFNS}_{f} \mathrm{~S}$ efficiently deals with the finite order grades of the alternatives according to the attributes. Therefore, the proposed model is the extension of $\mathrm{FNS}_{f} \mathrm{~S}$ (Akram et al. 2018), $\mathrm{IFNS}_{f} \mathrm{~S}$ (Akram et al. 2019b), $\mathrm{PFNS}_{f} \mathrm{~S}$ (Zhang et al. 1965), and $\mathrm{CPFNS}_{f} \mathrm{~S}$ (Akram et al. 2021e) models and in fact dominates overall traditional models of literature as it has comparatively wide range.

\subsection{Outline of the article}

The essence of the first part of this article is to propose the hybrid model of $\mathrm{CFFNS}_{f} \mathrm{Ss}$ and the related concepts including score function and accuracy function. Further, we investigate the remarkable properties and basic operations of $\mathrm{CFFNS}_{f} \mathrm{Ss}$. We have also constructed the $\mathrm{CFFNS}_{f} \mathrm{~S}$ derived by the threshold. Furthermore, algebraic and Yager operations for $\mathrm{CFFNS}_{f}$ numbers $\left(\mathrm{CFFNS}_{f} \mathrm{Ns}\right)$ are also defined. The proposed model is supported by the construction of three algorithms of decision-making and the applications are presented in contemplation of comparing the results of our algorithms. The comparative results of the model with existing $\mathrm{FFY}_{w} \mathrm{~A}$ (Garg et al. 2020) and $\mathrm{FFY}_{w} \mathrm{G}$ (Garg et al. 2020) operators are given in the paper.

On the other hand, we revamp the TOPSIS approach for the environment of $\mathrm{CFFNS}_{f}$ to account for MAGDM problems. The innovative $\mathrm{CFFNS}_{f}$ weighted average operator and the distance measure of alternatives from positive and negative ideal solutions are employed to examine the contiguity of optimal variables from ideal solutions. The accountability of the presented technique is illustrated by implementing its magnificent procedure to select the suitable city in the USA for farming. A comparative analysis with the existing FF-TOPSIS (Senapati and Yager 2020) approach has been demonstrated to endorse the phenomenal feasibility and viability of the set forth strategy. The merits of the developed model and TOPSIS approach are also narrated for the appropriate manifestation of its marvelous and incredible feasibility over the existing models and approaches.

We summarize the main contributions of our research work as follows: 
1. The article sets up a new theory of $\mathrm{CFFNS}_{f} \mathrm{~S}$ to deal with imprecise information involving vagueness and periodicity of ordered graded parameterized structure.

2. The algorithms are developed to tackle multi-attribute decision making problems by using numerical examples.

3. The comparative analysis with $\mathrm{FFY}_{w} \mathrm{~A}$ operator and FFY $_{w} \mathrm{G}$ operator to show the adequacy of the presented method.

4. This research also accomplishes a MAGDM technique, namely CFFNS $_{f}$-TOPSIS method.

5. The proposed technique is corroborated by a numerical example related to selecting the most suitable city in the USA for farming.

6. The $\mathrm{CFFNS}_{f}$-TOPSIS approach is dexterously accomplished by demonstrating a comparative analysis with FF-TOPSIS method.

\subsection{Layout of the paper}

From this point on, the paper is organized as follows. Section 2 contains some definitions of existing models. In Sect. 3, we introduce the novel concept of $\mathrm{CFFNS}_{f} \mathrm{~S}$ followed by operations on $\mathrm{CFFNS}_{f} \mathrm{Ss}$. Section 4 scrutinizes the algebraic and Yager operations on $\mathrm{CFFNS}_{f}$ Ns. Section 5 describes the three proposed algorithms of the decision-making process and also provides some applications of multi-variable decision-making procedures. Section 6 carries out a comparative analysis with existing models and offers experimental results that illustrate the effectiveness of the proposed algorithms. Then, Sect. 7 introduces the $\mathrm{CFFNS}_{f}$-TOPSIS method for MAGDM problems. A real example and a comparative study of its usefulness is shown in Sects. 8 and 9 . Finally, merits of the proposed model and conclusions are drawn in Sects. 10 and 11 .

\section{Preliminaries}

Definition 2.1 (Molodtsov (1999)) Let $U$ be a universe of discourse under consideration and $\mathcal{A}$ be the set of all attributes, $\mathcal{B} \subseteq \mathcal{A}$. A pair $(\rho, \mathcal{B})$ is called soft set over $U$ if $\rho: \mathcal{B} \longrightarrow P(U)$ where $\rho$ is a set-valued function.

Definition 2.2 (Fatimah et al. (2018)) Let $U$ be a universe of discourse and $\mathcal{A}$ be the set of all attributes, $\mathcal{B} \subseteq \mathcal{A}$. Consider $\mathcal{R}=\{0,1, \ldots, N-1\}$ be a set of ordered grades where $N \in\{2,3, \ldots\}$. A triple $(\mathcal{F}, \mathcal{B}, N)$ is an $\mathrm{NS}_{f} S$ on $U$ if $\mathcal{F}: \mathcal{B} \longrightarrow 2^{U \times \mathcal{R}}$, with the property that for each $b_{t} \in \mathcal{B}$ there exists a unique $\left(u_{g}, r_{a}\right) \in U \times \mathcal{R}$ such that $\left(u_{g}, r_{a}\right) \in \mathcal{F}\left(b_{t}\right), u_{g} \in U, r_{a_{g t}} \in \mathcal{R}$.
Definition 2.3 (Senapati and Yager (2020)) Consider $U$ be a universe of discourse. An FFS $E$ on $U$ is defined as an object of the form

$\mathfrak{\Im}=\left(\varrho_{E}, \varpi_{E}\right)=\left\{\left(u_{g}, \varrho_{E}\left(u_{g}\right), \varpi_{E}\left(u_{g}\right) \mid u_{g} \in U\right\}\right.$,

where the functions $\varrho_{E}: U \longrightarrow[0,1]$ and $\varpi_{E}: U \longrightarrow[0,1]$ denote the degree of membership (namely $\varrho_{E}\left(u_{g}\right)$ ) and the degree of non-membership (namely $\varpi_{E}\left(u_{g}\right)$ ) of the element $u_{g} \in U, \quad$ respectively, and for all $u_{g} \in$ $U, 0 \leq\left(\varrho_{E}\left(u_{g}\right)\right)^{3}+\left(\varpi_{E}\left(u_{g}\right)\right)^{3} \leq 1$. The value $\chi_{E}\left(u_{g}\right)=$ $\sqrt[3]{1-\left(\varrho_{E}\left(u_{g}\right)\right)^{3}-\left(\varpi_{E}\left(u_{g}\right)\right)^{3}}$ is called degree of uncertainty of the elements $u_{g} \in U$ to the FFS $E$.

Definition 2.4 A complex Fermatean fuzzy set (CFFS, in short) $\mathcal{B}$, defined on the universal set $U$, is characterized by the membership and non-membership functions $\mu_{\mathcal{B}}\left(u_{g}\right)$ and $v_{\mathcal{B}}\left(u_{g}\right)$, respectively, which assign to each element $u_{g} \in U$ a complex-valued grade of membership and nonmembership functions in $\mathcal{B}$. The CFFS may be represented as the set of triples:

$\mathcal{B}=\left\{\left\langle u_{g}, \mu_{\mathcal{B}}\left(u_{g}\right), v_{\mathcal{B}}\left(u_{g}\right)\right\rangle: u_{g} \in U\right\}$,

where $\mu_{\mathcal{B}}\left(u_{g}\right): U \longrightarrow\left\{u_{g}\left|u_{g} \in \mathbb{C},\right| u_{g} \mid \leq 1\right\}, \quad v_{\mathcal{B}}\left(u_{g}\right):$ $U \longrightarrow\left\{u_{g}^{\prime}\left|u_{g}^{\prime} \in \mathbb{C},\right| u_{g} \mid \leq 1\right\}$, such that $\mu_{\mathcal{B}}\left(u_{g}\right)=$ $s_{\mathcal{B}}\left(u_{g}\right) e^{i \omega_{\mathcal{B}}\left(u_{g}\right)}, v_{\mathcal{B}}\left(u_{g}\right)=k_{\mathcal{B}}\left(u_{g}\right) e^{i \psi_{\mathcal{B}}\left(u_{g}\right)}$, where $i=\sqrt{-1}$ and $s_{\mathcal{B}}\left(u_{g}\right), k_{\mathcal{B}}\left(u_{g}\right), \omega_{\mathcal{B}}\left(u_{g}\right), \psi_{\mathcal{B}}\left(u_{g}\right)$ are real-valued functions such that $s_{\mathcal{B}}\left(u_{g}\right), k_{\mathcal{B}}\left(u_{g}\right) \in[0,1], \omega_{\mathcal{B}}\left(u_{g}\right), \psi_{\mathcal{B}}\left(u_{g}\right) \in[0,2 \pi]$. $s_{\mathcal{B}}\left(u_{g}\right), k_{\mathcal{B}}\left(u_{g}\right)$ are called the amplitude terms and $\omega_{\mathcal{B}}\left(u_{g}\right), \psi_{\mathcal{B}}\left(u_{g}\right)$ are called the phase terms with $0 \leq$ $\left(s_{\mathcal{B}}\left(u_{g}\right)\right)^{3}+\left(k_{\mathcal{B}}\left(u_{g}\right)\right)^{3} \leq 1$, and $0 \leq\left(\frac{\omega_{\mathcal{B}}\left(u_{g}\right)}{2 \pi}\right)^{3}+\left(\frac{\psi_{\mathcal{B}}\left(u_{g}\right)}{2 \pi}\right)^{3} \leq 1$.

The term $\pi_{\mathcal{B}}\left(u_{g}\right)=j_{\mathcal{B}}\left(u_{g}\right) e^{i 2 \pi \vartheta_{\mathcal{B}}\left(u_{g}\right)}$ is called degree of indeterminacy, $\quad$ where $\quad j_{\mathcal{B}}\left(u_{g}\right)=$ $\sqrt[3]{1-\left(s_{\mathcal{B}}\left(u_{g}\right)\right)^{3}-\left(k_{\mathcal{B}}\left(u_{g}\right)\right)^{3}} \quad$ and $\quad \vartheta_{\mathcal{B}}=$ $\sqrt[3]{1-\left(\frac{\omega_{\mathcal{B}}\left(u_{g}\right)}{2 \pi}\right)^{3}-\left(\frac{\psi_{\mathcal{B}}\left(u_{g}\right)}{2 \pi}\right)^{3}}$.

Definition 2.5 Let $U$ be a universal set and $\mathcal{A}$ be the set of all attributes under consideration, $\mathcal{B} \subseteq \mathcal{A}$. Let $\mathcal{P}(U)$ denotes the set of all complex Fermatean fuzzy (CFF) subsets of $U$. A pair $(\mathcal{P}, \mathcal{B})$ is called a $\operatorname{CFFS}_{f} S$ over $U$, where $\mathcal{P}$ is a function given by $\mathcal{P}: \mathcal{B} \longrightarrow \mathcal{P}(U)$, which is $\mathcal{P}_{e_{\mathcal{P}}}\left(b_{t}\right)=\left\{\left\langle u_{g}, s_{\mathcal{P}}\left(u_{g}\right) e^{i \omega_{\mathcal{P}}\left(u_{g}\right)}, k_{\mathcal{P}} e^{i \psi_{\mathcal{P}}\left(u_{g}\right)}\right\rangle \mid u_{g} \in U, b_{t} \in \mathcal{B}\right\}$. 


\section{Complex Fermatean fuzzy $\mathbf{N}$-soft sets}

Definition 3.1 Let $U$ be a universal set and $\mathcal{A}$ be the set of all parameters under consideration, $\mathcal{B} \subseteq \mathcal{A}$. Let $\mathcal{R}=$ $\{0,1,2, \ldots, N-1\}$ be a set of ordered grades where $N \in$ $\{2,3, \ldots\}$. A triple $(\mathcal{H}, \mathcal{Q}, N)$ is called a $C F F N$-soft set (for short, $\mathrm{CFFNS}_{f} \mathrm{~S}$ ), when $\mathcal{Q}=(\mathcal{F}, \mathcal{B}, N)$ is an $\mathrm{NS}_{f} \mathrm{~S}$ on $U$ and $\mathcal{H}$ is a mapping $\mathcal{H}: \mathcal{B} \longrightarrow C F F^{(U \times \mathcal{R})}$, where $C F F^{(U \times \mathcal{R})}$ is the collection of all CFFSs over $U \times \mathcal{R}$, which is
Example 3.1 Consider that an auto broker decides to purchase the car from auto company. The best car is chosen by spade ratings endowed by an expert. These rankings are on the basis of launched cars in the last 5 years and their performances. Before purchasing the car, auto broker obtained some rating and ranking based information from an expert about four different models of vehicles having different manufacturing dates. Let $X=\left\{x_{1}, x_{2}, x_{3}, x_{4}\right\}$ be the set of vehicles and $\mathcal{B}=\left\{b_{1}=\right.$ Reliability, $b_{2}=$ Maximumpayload, $b_{3}=$ Purchasingcost $\} \subseteq \mathcal{A}$ be the set of

$\mathcal{H}\left(b_{t}\right)=\left\{\left\langle\left(u_{g}, r_{a_{g t}}\right), s\left(u_{g}, r_{a_{g t}}\right) e^{i \omega\left(u_{g}, r_{a_{g t}}\right)}, k\left(u_{g}, r_{a_{g t}}\right) e^{i \psi\left(u_{g}, r_{a_{g t}}\right)}\right\rangle \mid b_{t} \in \mathcal{B},\left(u_{g}, r_{a_{g t}}\right) \in U \times \mathcal{R}\right\}$.

For convenience, $\mathcal{H}\left(b_{t}\right)=\left\langle\left(u_{g}, r_{a_{g t}}\right), s\left(u_{g}, r_{a_{g t}}\right) e^{i \omega\left(u_{g}, r_{a_{g t}}\right)}\right.$, $\left.k\left(u_{g}, r_{a_{g t}}\right) e^{i \psi\left(u_{g}, r_{a_{g t}}\right)}\right\rangle \quad$ is denoted by $\quad \alpha_{a_{g t}}=\left\langle r_{a_{g t}}\right.$, $\left.\left(s_{a_{g t}} e^{i \omega_{a_{g t}}}, k_{a_{g t}} e^{i \psi_{a_{g t} t}}\right)\right\rangle$ which represents $\mathrm{CFFNS}_{f}$ number $\left(\mathrm{CFFNS}_{f} \mathrm{~N}\right)$.

Definition 3.2 Let $\alpha_{a_{g t}}=\left\langle r_{a_{g t}},\left(s_{a_{g t}} e^{i \omega_{a_{g t}}}, k_{a_{g t}} e^{i \psi_{a_{g t}}}\right)\right\rangle$ be a $\mathrm{CFFNS}_{f} \mathrm{~N}$ then

$$
\Lambda_{\alpha_{a_{g t}}}=\sqrt[3]{1-\left(\left(s_{a_{g t}}\right)^{3}+\left(k_{a_{g t}}\right)^{3}\right)} e^{i 2 \pi} \sqrt[3]{1-\left(\left(\frac{\omega_{a_{g t}}}{2 \pi}\right)^{3}+\left(\frac{\psi_{a_{g t}}}{2 \pi}\right)^{3}\right)}
$$

is called the degree of hesitancy/indeterminacy of $\mathrm{CFFNS}_{f} \mathrm{~N}$.

Definition 3.3 Let $\alpha_{a_{g t}}=\left\langle r_{a_{g t}},\left(s_{a_{g t}} e^{i \omega_{a_{g t}}}, k_{a_{g t}}, e^{i \psi_{a_{g t} t}}\right)\right\rangle$ be any $\mathrm{CFFNS}_{f} \mathrm{~N}$ over $U$. The score function and accuracy function of $\alpha_{a_{g t}}$ are defined as follows:

$$
\begin{aligned}
& S\left(\alpha_{a_{g t}}\right)=\left(\frac{r_{a_{g t}}}{N-1}\right)^{3}+\left(s_{a_{g t}}\right)^{3}-\left(k_{a_{g t}}\right)^{3}+\left(\left(\frac{\omega_{a_{g t}}}{2 \pi}\right)^{3}-\left(\frac{\psi_{a_{g t}}}{2 \pi}\right)^{3}\right), \\
& A\left(\alpha_{a_{g t}}\right)=\left(\frac{r_{a_{g t}}}{N-1}\right)^{3}+\left(s_{a_{g t}}\right)^{3}+\left(k_{a_{g t}}\right)^{3}+\left(\left(\frac{\omega_{a_{g t}}}{2 \pi}\right)^{3}+\left(\frac{\psi_{a_{g t}}}{2 \pi}\right)^{3}\right),
\end{aligned}
$$

respectively, where $S\left(\alpha_{a_{g t}}\right) \in[-2,3]$ and $A\left(\alpha_{a_{g t}}\right) \in[0,3]$.

Definition 3.4 For any two distinct $\mathrm{CFFNS}_{f} \mathrm{Ns} \alpha_{a_{g t}}$ and $\beta_{a_{l t}}$, we have:

1. if $S\left(\alpha_{a_{g t}}\right)<S\left(\beta_{a_{l t}}\right)$, then $\alpha_{a_{g t}}<\beta_{a_{l t}}$,

2. if $S\left(\alpha_{a_{g t}}\right)>S\left(\beta_{a_{l t}}\right)$, then $\alpha_{a_{g t}}>\beta_{a_{l t}}$,

3. if $S\left(\alpha_{a_{g t}}\right)=S\left(\beta_{a_{l t}}\right)$, then
a. if $A\left(\alpha_{a_{g t}}\right)>A\left(\beta_{a_{l t}}\right)$, then $\alpha_{a_{g t}}>\beta_{a_{l t}}$,
b. if $A\left(\alpha_{a_{g t}}\right)<A\left(\beta_{a_{l t}}\right)$, then $\alpha_{a_{g t}}<\beta_{a_{l t}}$,
c. if $A\left(\alpha_{a_{g t}}\right)=A\left(\beta_{a_{l t}}\right)$, then $\alpha_{a_{g t}}=\beta_{a_{l t}}$.

For a better understanding of the concept of our new model, we present the following example: attributes, that are used to set grades for each vehicle with respect to each attribute. The expert assigned the rating of the cars according to the above-mentioned conflicting criteria and the initial review recapped in Table 3, where:

- four spades represent 'excellent',

- three spades represent 'very good',

- two spades represent 'good',

- one spade represents 'regular', and

- a bullet represents 'bad'.

The set of grades $\mathcal{R}=\{0,1,2,3,4\}$ can be easily associated with rated assessment conducted by bullet and spades as follows:

- 0 stands for ' $\bullet$ ',

- 1 stands for ' $\boldsymbol{\Lambda}$ ',

- 2 stands for 'As',

- 3 stands for 'MAC',

- 4 stands for 'MAM'.

Based on the overall qualities of the cars, the auto broker

\begin{tabular}{|c|c|c|c|}
\hline$X / \mathcal{B}$ & $b_{1}$ & $b_{2}$ & $b_{3}$ \\
\hline$x_{1}$ & A & AMA & A \\
\hline$x_{2}$ & $\boldsymbol{\phi}$ & AMA & A \\
\hline$x_{3}$ & AAM & AMAM & $\bullet$ \\
\hline$x_{4}$ & $\bullet$ & A & AM \\
\hline
\end{tabular}
gives evaluation scores of the cars which is shown as

Table 3 Information extracted from the expert 
Table 3 and the tabular representation of its associated 5 -soft set is given in Table 4.

The grade data in the actual information can be easily extracted. However, according to the Definition 3.1 when the data possess fuzzy uncertainty characteristics, we need $\mathrm{CFFNS}_{f} \mathrm{~N}$. It provides us information in which the auto brokers evaluate the cars and specify their rankings based on the same multiple fuzzy characteristics from the perspective of the two-dimensional membership degree and non-membership degree. This assessment of cars by auto brokers complies with the guidelines as follows:

$-2.0 \leq S(X)<-1.2$ when grade 0,

$-1.2 \leq S(X)<-0.4$ when grade 1 ,

$-0.4 \leq S(X)<0.4$ when grade 2 ,

$0.4 \leq S(X)<1.2$ when grade 3 ,

$1.2 \leq S(X)<2.0$ when grade 4 .

According to above criteria, we can obtain Table 5.

Therefore, by Definition 3.1, the $\mathrm{CFF}_{5} \mathrm{~S}_{f} \mathrm{~S}(\mathcal{H}, \mathcal{Q}, 5)$ can be defined as follows:
The $\mathrm{CFF}_{5 \mathrm{~S}} \mathrm{~S}(\mathcal{H}, \mathcal{Q}, 5)$ can be represented more clearly in tabular form shown as in Table 6 as follows:

Remark 1 The following observations are in order:

1. In Example 3.1, we consider the five assessment grades, but the assessment grades in practical problems do not necessarily utilize the 5 grades, it can be arbitrary. Generally, the range concerning the score function of CFF numbers can vary with actual grade requirements.

2. Any $\mathrm{CFF}_{2} \mathrm{~S}_{f} \mathrm{~S}(\mathcal{H}, \mathcal{Q}, 2)$ can be naturally associated with a $\mathrm{CFFS}_{f} \mathrm{~S}$. We identify a $\mathrm{CFF}_{2} \mathrm{~S}_{f} \mathrm{~S} \mathcal{H}: \mathcal{B} \longrightarrow$ $C F F^{(U \times\{0,1\})}$ with a $\operatorname{CFFS}_{f} \mathrm{~S}(\wp, \mathcal{B})$, which is given by:

$$
\begin{gathered}
\wp\left(b_{t}\right)=\left\{\left\langle u_{g}, s_{\wp}\left(u_{g}\right) e^{i \omega_{\wp}\left(u_{g}\right)}, k_{\wp}\left(u_{g}\right) e^{i \psi_{\wp}\left(u_{g}\right)}\right\rangle \mid\left\langle\left(u_{g}, 1\right),\right.\right. \\
\left.\left.s_{\wp}\left(u_{g}\right) e^{i \omega_{\wp}\left(u_{g}\right)}, k_{\wp}\left(u_{g}\right) e^{i \psi_{\wp}\left(u_{g}\right)}\right\rangle \in \mathcal{H}\left(b_{t}\right)\right\},
\end{gathered}
$$

for every $b_{t} \in \mathcal{B}$, where $C F F^{(U \times\{0,1\})}$ is the collection of all CFF subsets of $U \times\{0,1\}$.

3. An arbitrary $\mathrm{CFFNS}_{f} \mathrm{~S}$ over a universe $U$ can be identified as a CFF $(N+1)$-soft set. For example, from

$$
\begin{aligned}
h\left(b_{1}\right)= & \left\{\left\langle\left(x_{1}, 2\right), 0.5 e^{i 0.9 \pi}, 0.6 e^{i 1.2 \pi}\right\rangle,\left\langle\left(x_{2}, 1\right), 0.3 e^{i 0.5 \pi}, 0.7 e^{i 1.5 \pi}\right\rangle,\left\langle\left(x_{3}, 3\right), 0.7 e^{i 1.4 \pi}, 0.3 e^{i 0.5 \pi}\right\rangle,\right. \\
& \left.\left\langle\left(x_{4}, 0\right), 0.1 e^{i 0.2 \pi}, 0.9 e^{i 1.8 \pi}\right\rangle\right\}, \\
h\left(b_{2}\right)= & \left\{\left\langle\left(x_{1}, 3\right), 0.7 e^{i 1.5 \pi}, 0.3 e^{i 0.5 \pi}\right\rangle,\left\langle\left(x_{2}, 3\right), 0.8 e^{i 1.6 \pi}, 0.4 e^{i 0.6 \pi}\right\rangle,\left\langle\left(x_{3}, 4\right), 0.9 e^{i 1.8 \pi}, 0.1 e^{i 0.3 \pi}\right\rangle,\right. \\
& \left.\left\langle\left(x_{4}, 2\right), 0.5 e^{i 0.9 \pi}, 0.5 e^{i 1.0 \pi}\right\rangle\right\}, \\
h\left(b_{3}\right)= & \left\{\left\langle\left(x_{1}, 1\right), 0.3 e^{i 0.6 \pi}, 0.8 e^{i 1.6 \pi}\right\rangle,\left\langle\left(x_{2}, 2\right), 0.6 e^{i 0.9 \pi}, 0.5 e^{i 1.2 \pi}\right\rangle,\left\langle\left(x_{3}, 0\right), 0.1 e^{i 0.2 \pi}, 0.9 e^{i 1.9 \pi}\right\rangle,\right. \\
& \left.\left\langle\left(x_{4}, 4\right), 0.9 e^{i 1.9 \pi}, 0.2 e^{i 0.3 \pi}\right\rangle\right\} .
\end{aligned}
$$

Table 4 Tabular form of the 5-soft set

\begin{tabular}{llll}
\hline$(\mathcal{F}, \mathcal{B}, 5)$ & $b_{1}$ & $b_{2}$ & $b_{3}$ \\
\hline$x_{1}$ & 2 & 3 & 1 \\
$x_{2}$ & 1 & 3 & 2 \\
$x_{3}$ & 3 & 4 & 0 \\
$x_{4}$ & 0 & 2 & 4 \\
\hline
\end{tabular}

Table 5 Grading criteria

\begin{tabular}{llllll}
\hline$r_{a_{g t}} / \mathcal{H}$ & \multicolumn{2}{l}{ Amplitude term } & & Phase term & \\
\cline { 2 - 3 } \cline { 5 - 6 } Grades & $s_{a_{g t}}$ & $k_{a_{g t}}$ & & $\omega_{a_{g t}}$ & $\psi_{a_{g t}}$ \\
\hline$r_{a_{g t}}=0$ & {$[0,0.2)$} & $(0.85,1]$ & {$[0,0.4 \pi)$} & $(1.7 \pi, 2 \pi]$ \\
$r_{a_{g t}}=1$ & {$[0.2,0.4)$} & $(0.65,0.85]$ & {$[0.4 \pi, 0.8 \pi)$} & $(1.3 \pi, 1.7 \pi]$ \\
$r_{a_{g t}}=2$ & {$[0.4,0.65)$} & $(0.4,0.65]$ & {$[0.8 \pi, 1.3 \pi)$} & $(0.8 \pi, 1.3 \pi]$ \\
$r_{a_{g t}}=3$ & {$[0.65,0.85)$} & $(0.2,0.4]$ & {$[1.3 \pi, 1.7 \pi)$} & $(0.4 \pi, 0.8 \pi]$ \\
$r_{a_{g t}}=4$ & {$[0.85,1)$} & $(0,0.2]$ & {$[1.7 \pi, 2 \pi)$} & $(0,0.4 \pi]$ \\
\hline
\end{tabular}

Table 6, a $\mathrm{CFF}_{5} \mathrm{~S}(\mathcal{H}, \mathcal{Q}, 5)$ can be identified as a $\mathrm{CFF}_{6} \mathrm{~S}$ over $U$. In a $\mathrm{CFF}_{6} \mathrm{~S}$ S, we consider that there is a 5 grade, which is never used in Example 3.1.

4. In Definition 3.1, grade 0 describes the lowest score. It does not mean that there is incomplete information or no assessment.

Definition 3.5 A $\mathrm{CFFNS}_{f} \mathrm{~S}(\mathcal{H}, \mathcal{Q}, N)$ over universe of discourse $U$, where $\mathcal{Q}=(\mathcal{F}, \mathcal{B}, N)$ is an $\mathrm{NS}_{f} \mathrm{~S}$, is said to be efficient if $\mathcal{H}\left(b_{t}\right)=\left\langle\left(u_{g}, N-1\right), 1 e^{i 2 \pi}, 0 e^{i 0 \pi}\right\rangle$ for some $b_{t} \in \mathcal{B}, u_{g} \in U$.

Example 3.2 By inspection, it can be checked that the $\mathrm{CFF}_{5} \mathrm{~S}_{f} \mathrm{~S}$ defined in Example 3.1 is not efficient. However, $\mathrm{CFF}_{5} \mathrm{~S}(\mathcal{H}, \mathcal{Q}, 5)$ in Table 7 is efficient.

Definition 3.6 Let $\left(\mathcal{H}_{1}, \mathcal{Q}_{1}, N_{1}\right)$ and $\left(\mathcal{H}_{2}, \mathcal{Q}_{2}, N_{2}\right)$ be two $\mathrm{CFFNS}_{f}$ Ss over universe of discourse $U$, where $\mathcal{Q}_{1}=$ $\left(\mathcal{F}_{1}, \mathcal{B}_{1}, N_{1}\right), \mathcal{Q}_{2}=\left(\mathcal{F}_{2}, \mathcal{B}_{2}, N_{2}\right) \quad$ are $\quad \mathrm{NS}_{f} \mathrm{Ss}$, then 
Table 6 Tabular form of the $\mathrm{CFF}_{5} \mathrm{~S} S(\mathcal{H}, \mathcal{Q}, 5)$

\begin{tabular}{llll}
\hline$(\mathcal{H}, \mathcal{Q}, 5)$ & $b_{1}$ & $b_{2}$ & $b_{3}$ \\
\hline$x_{1}$ & $\left\langle 2,\left(0.5 e^{i 0.9 \pi}, 0.6 e^{i 1.2 \pi}\right)\right\rangle$ & $\left\langle 3,\left(0.7 e^{i 1.5 \pi}, 0.3 e^{i 0.5 \pi}\right)\right\rangle$ & $\left\langle 1,\left(0.3 e^{i 0.6 \pi}, 0.8 e^{i 1.6 \pi}\right)\right\rangle$ \\
$x_{2}$ & $\left\langle 1,\left(0.3 e^{i 0.5 \pi}, 0.7 e^{i 1.5 \pi}\right)\right\rangle$ & $\left\langle 3,\left(0.8 e^{i 1.6 \pi}, 0.4 e^{i 0.6 \pi}\right)\right\rangle$ & $\left\langle 2,\left(0.6 e^{i 0.9 \pi}, 0.5 e^{i 1.2 \pi}\right)\right\rangle$ \\
$x_{3}$ & $\left\langle 3,\left(0.7 e^{i 1.4 \pi}, 0.3 e^{i 0.5 \pi}\right)\right\rangle$ & $\left\langle 4,\left(0.9 e^{i 1.8 \pi}, 0.1 e^{i 0.3 \pi}\right)\right\rangle$ & $\left\langle 0,\left(0.1 e^{i 0.2 \pi}, 0.9 e^{i 1.9 \pi}\right)\right\rangle$ \\
$x_{4}$ & $\left\langle 0,\left(0.1 e^{i 0.2 \pi}, 0.9 e^{i 1.8 \pi}\right)\right\rangle$ & $\left\langle 2,\left(0.5 e^{i 0.9 \pi}, 0.5 e^{i 1.0 \pi}\right)\right\rangle$ & $\left\langle 4,\left(0.9 e^{i 1.9 \pi}, 0.2 e^{i 0.3 \pi}\right)\right\rangle$ \\
\hline
\end{tabular}

\begin{tabular}{llll}
\hline$(\mathcal{H}, \mathcal{Q}, 5)$ & $b_{1}$ & $b_{2}$ & $b_{3}$ \\
\hline$x_{1}$ & $\left\langle 2,\left(0.5 e^{i 0.9 \pi}, 0.6 e^{i 1.2 \pi}\right)\right\rangle$ & $\left\langle 3,\left(0.7 e^{i 1.5 \pi}, 0.3 e^{i 0.5 \pi}\right)\right\rangle$ & $\left\langle 1,\left(0.3 e^{i 0.6 \pi}, 0.8 e^{i 1.6 \pi}\right)\right\rangle$ \\
$x_{2}$ & $\left\langle 1,\left(0.3 e^{i 0.5 \pi}, 0.7 e^{i 1.5 \pi}\right)\right\rangle$ & $\left\langle 3,\left(0.8 e^{i 1.6 \pi}, 0.4 e^{i 0.6 \pi}\right)\right\rangle$ & $\left\langle 2,\left(0.6 e^{i 0.9 \pi}, 0.5 e^{i 1.2 \pi}\right)\right\rangle$ \\
$x_{3}$ & $\left\langle 3,\left(0.7 e^{i 1.4 \pi}, 0.3 e^{i 0.5 \pi}\right)\right\rangle$ & $\left\langle 4,\left(0.9 e^{i 1.8 \pi}, 0.1 e^{i 0.3 \pi}\right)\right\rangle$ & $\left\langle 0,\left(0.1 e^{i 0.2 \pi}, 0.9 e^{i 1.9 \pi}\right)\right\rangle$ \\
$x_{4}$ & $\left\langle 0,\left(0.1 e^{i 0.2 \pi}, 0.9 e^{i 1.8 \pi}\right)\right\rangle$ & $\left\langle 2,\left(0.5 e^{i 0.9 \pi}, 0.5 e^{i 1.0 \pi}\right)\right\rangle$ & $\left\langle 4,\left(1.0 e^{i 2.0 \pi}, 0.0 e^{i 0.0 \pi}\right)\right\rangle$ \\
\hline
\end{tabular}

Table 7 Tabular form of the efficient $\mathrm{CFF}_{5} \mathrm{~S}(\mathcal{H}, \mathcal{Q}, 5)$
In $\mathrm{CFF}$ complement, the grades are same as in the original $\mathrm{NS}_{f} \mathrm{~S}$, however all their membership and nonmembership degrees are complementary.

Definition 3.9 Let $(\mathcal{H}, \mathcal{Q}, N)$ be a $\mathrm{CFFNS}_{f} \mathrm{~S}$ over universe of discourse $U$, where $\mathcal{Q}=(\mathcal{F}, \mathcal{B}, N)$ is an $\mathrm{NS}_{f} \mathrm{~S}$, then $\left(\mathcal{H}^{c}, \mathcal{Q}^{c}, N\right)$ is said to be weak CFF complement when $\left(\mathcal{H}, \mathcal{Q}^{c}, N\right)$ is a weak complement and $\left(\mathcal{H}^{c}, \mathcal{Q}, N\right)$ is a $\mathrm{CFF}$ complement.

In other words, a weak CFF complement of $\mathrm{CFFNS}_{f} \mathrm{~S}$ is the CFF complement of any of its weak complement.

Example 3.3 A weak complement $\left(\mathcal{H}, \mathcal{Q}^{c}, 5\right)$ of $\mathrm{CFF}_{5} \mathrm{~S}_{f} \mathrm{~S}$ in Example 3.1, Table 6, is represented by Table 8 . The CFF complement of this $\mathrm{CFF}_{5} \mathrm{~S} S$ is $\left(\mathcal{H}^{c}, \mathcal{Q}, 5\right)$ defined by Table 9. A weak CFF complement of this $\mathrm{CFF}_{5} \mathrm{~S}$ is $\left(\mathcal{H}^{c}, \mathcal{Q}^{c}, 5\right)$ defined by Table 10.

Definition 3.10 For a $\operatorname{CFFNS}_{f} \mathrm{~S}(\mathcal{H}, \mathcal{Q}, N)$, where $\mathcal{Q}=$ $(\mathcal{F}, \mathcal{B}, N)$ is an $\mathrm{NS}_{f} \mathrm{~S}$, the top weak complement of $(\mathcal{H}, \mathcal{Q}, N)$ is $\left(\mathcal{H}, \mathcal{Q}^{>}, N\right)$, and the top weak CFF complement of $(\mathcal{H}, \mathcal{Q}, N)$ is $\left(\mathcal{H}^{c}, \mathcal{Q}^{>}, N\right)$, where $\mathcal{Q}^{>}=$ $\left(\mathcal{F}^{>}, \mathcal{B}, N\right)$ is the top weak complement of $\mathcal{Q}=(\mathcal{F}, \mathcal{B}, N)$ and defined as follows:
Table 8 A weak complement of the $\mathrm{CFF}_{5} \mathrm{~S}(\mathcal{H}, \mathcal{Q}, 5)$ in Example 3.1

\begin{tabular}{llll}
\hline$\left(H, \mathcal{Q}^{c}, 5\right)$ & $b_{1}$ & $b_{2}$ & $b_{3}$ \\
\hline$x_{1}$ & $\left\langle 3,\left(0.5 e^{i 0.9 \pi}, 0.6 e^{i 1.2 \pi}\right)\right\rangle$ & $\left\langle 4,\left(0.7 e^{i 1.5 \pi}, 0.3 e^{i 0.5 \pi}\right)\right\rangle$ & $\left\langle 2,\left(0.3 e^{i 0.6 \pi}, 0.8 e^{i 1.6 \pi}\right)\right\rangle$ \\
$x_{2}$ & $\left\langle 0,\left(0.3 e^{i 0.5 \pi}, 0.7 e^{i 1.5 \pi}\right)\right\rangle$ & $\left\langle 2,\left(0.8 e^{i 1.6 \pi}, 0.4 e^{i 0.6 \pi}\right)\right\rangle$ & $\left\langle 3,\left(0.6 e^{i 0.9 \pi}, 0.5 e^{i 1.2 \pi}\right)\right\rangle$ \\
$x_{3}$ & $\left\langle 2,\left(0.7 e^{i 1.4 \pi}, 0.3 e^{i 0.5 \pi}\right)\right\rangle$ & $\left\langle 3,\left(0.9 e^{i 1.8 \pi}, 0.1 e^{i 0.3 \pi}\right)\right\rangle$ & $\left\langle 2,\left(0.1 e^{i 0.2 \pi}, 0.9 e^{i 1.9 \pi}\right)\right\rangle$ \\
$x_{4}$ & $\left\langle 4,\left(0.1 e^{i 0.2 \pi}, 0.9 e^{i 1.8 \pi}\right)\right\rangle$ & $\left\langle 1,\left(0.5 e^{i 0.9 \pi}, 0.5 e^{i 1.0 \pi}\right)\right\rangle$ & $\left\langle 3,\left(0.9 e^{i 1.9 \pi}, 0.2 e^{i 0.3 \pi}\right)\right\rangle$ \\
\hline
\end{tabular}


Table 9 Tabular representation of the CFF complement of the $\mathrm{CFF}_{5} \mathrm{~S} S(\mathcal{H}, \mathcal{Q}, 5)$ in Example 3.1

\begin{tabular}{llll}
\hline$\left(H^{c}, \mathcal{Q}, 5\right)$ & $b_{1}$ & $b_{2}$ & $b_{3}$ \\
\hline$x_{1}$ & $\left\langle 2,\left(0.6 e^{i 1.2 \pi}, 0.5 e^{i 0.9 \pi}\right)\right\rangle$ & $\left\langle 3,\left(0.3 e^{i 0.5 \pi}, 0.7 e^{i 1.5 \pi}\right)\right\rangle$ & $\left\langle 1,\left(0.8 e^{i 1.6 \pi}, 0.3 e^{i 0.6 \pi}\right)\right\rangle$ \\
$x_{2}$ & $\left\langle 1,\left(0.7 e^{i 1.5 \pi}, 0.3 e^{i 0.5 \pi}\right)\right\rangle$ & $\left\langle 3,\left(0.4 e^{i 0.6 \pi}, 0.8 e^{i 1.6 \pi}\right)\right\rangle$ & $\left\langle 2,\left(0.5 e^{i 1.2 \pi}, 0.6 e^{i 0.9 \pi}\right)\right\rangle$ \\
$x_{3}$ & $\left\langle 3,\left(0.3 e^{i 0.5 \pi}, 0.7 e^{i 1.4 \pi}\right)\right\rangle$ & $\left\langle 4,\left(0.1 e^{i 0.3 \pi}, 0.9 e^{i 1.8 \pi}\right)\right\rangle$ & $\left\langle 0,\left(0.9 e^{i 1.9 \pi}, 0.1 e^{i 0.2 \pi}\right)\right\rangle$ \\
$x_{4}$ & $\left\langle 0,\left(0.9 e^{i 1.8 \pi}, 0.1 e^{i 0.2 \pi}\right)\right\rangle$ & $\left\langle 2,\left(0.5 e^{i 1.0 \pi}, 0.5 e^{i 0.9 \pi}\right)\right\rangle$ & $\left\langle 4,\left(0.2 e^{i 0.3 \pi}, 0.9 e^{i 1.9 \pi}\right)\right\rangle$ \\
\hline
\end{tabular}

Table 10 Tabular representation of a weak CFF complement of the $\mathrm{CFF}_{\mathrm{S}} \mathrm{S}(\mathcal{H}, \mathcal{Q}, 5)$ in Example 3.1

\begin{tabular}{llll}
\hline$\left(H^{c}, \mathcal{Q}^{c}, 5\right)$ & $b_{1}$ & $b_{2}$ & $b_{3}$ \\
\hline$x_{1}$ & $\left\langle 3,\left(0.6 e^{i 1.2 \pi}, 0.5 e^{i 0.9 \pi}\right)\right\rangle$ & $\left\langle 4,\left(0.3 e^{i 0.5 \pi}, 0.7 e^{i 1.5 \pi}\right)\right\rangle$ & $\left\langle 2,\left(0.8 e^{i 1.6 \pi}, 0.3 e^{i 0.6 \pi}\right)\right\rangle$ \\
$x_{2}$ & $\left\langle 0,\left(0.7 e^{i 1.5 \pi}, 0.3 e^{i 0.5 \pi}\right)\right\rangle$ & $\left\langle 2,\left(0.4 e^{i 0.6 \pi}, 0.8 e^{i 1.6 \pi}\right)\right\rangle$ & $\left\langle 3,\left(0.5 e^{i 1.2 \pi}, 0.6 e^{i 0.9 \pi}\right)\right\rangle$ \\
$x_{3}$ & $\left\langle 2,\left(0.3 e^{i 0.5 \pi}, 0.7 e^{i 1.4 \pi}\right)\right\rangle$ & $\left\langle 3,\left(0.1 e^{i 0.3 \pi}, 0.9 e^{i 1.8 \pi}\right)\right\rangle$ & $\left\langle 2,\left(0.9 e^{i 1.9 \pi}, 0.1 e^{i 0.2 \pi}\right)\right\rangle$ \\
$x_{4}$ & $\left\langle 4,\left(0.9 e^{i 1.8 \pi}, 0.1 e^{i 0.2 \pi}\right)\right\rangle$ & $\left\langle 1,\left(0.5 e^{i 1.0 \pi}, 0.5 e^{i 0.9 \pi}\right)\right\rangle$ & $\left\langle 3,\left(0.2 e^{i 0.3 \pi}, 0.9 e^{i 1.9 \pi}\right)\right\rangle$ \\
\hline
\end{tabular}

et al.

2018)

$=$

$$
\begin{aligned}
& \left(\mathcal{H}, \mathcal{Q}^{<}, N\right)= \\
& \left\{\begin{array}{cl}
\left\langle 0,\left(s_{a_{g t}} e^{i \omega_{a_{g t}}}, k_{a_{g t}} e^{i \psi_{a_{g t} t}}\right)\right\rangle, & \text { if } r_{a_{g t}}>0, \\
\left\langle N-1,\left(s_{a_{g t}} e^{i \omega_{a_{g t}}}, k_{a_{g t}} e^{i \psi_{a_{g t}}}\right)\right\rangle, & \text { if } r_{a_{g t}}=0 .
\end{array}\right. \\
& \left(\mathcal{H}^{c}, \mathcal{Q}^{<}, N\right)= \\
& \left\{\begin{array}{cl}
\left\langle 0,\left(k_{a_{g t}} e^{i \psi_{a_{g t}}}, s_{a_{g t}} e^{i \omega_{a_{g t}}}\right)\right\rangle, & \text { if } r_{a_{g t}}>0, \\
\left\langle N-1,\left(k_{a_{g t}} e^{i \psi_{a_{g t} t}}, s_{a_{g t}} e^{i \omega_{a_{g t}}}\right)\right\rangle, & \text { if } r_{a_{g t}}=0 .
\end{array}\right.
\end{aligned}
$$

Example 3.5 The bottom weak complement and the bottom weak CFF complement of the $\mathrm{CFF} \mathrm{S}_{f} \mathrm{~S}$ Table 6 in Example 3.1 are given by Tables 13 and 14 .

Definition 3.12 Let $U$ be a universe of discourse and $\left(\mathcal{H}_{1}, \mathcal{Q}_{1}, N_{1}\right)$ and $\left(\mathcal{H}_{2}, \mathcal{Q}_{2}, N_{2}\right)$ be two CFFNS $_{f}$ Ss over nonempty set $U$, where $\mathcal{Q}_{1}=\left(\mathcal{F}_{1}, \mathcal{B}_{1}, N_{1}\right)$ and $\mathcal{Q}_{2}=$ $\left(\mathcal{F}_{2}, \mathcal{B}_{2}, N_{2}\right)$ are $\mathrm{NS}_{f} \mathrm{Ss}$ on $U$, then their restricted intersection is denoted by $\left(\mathcal{H}_{1}, \mathcal{Q}_{1}, N_{1}\right) \cap_{\mathfrak{R}}\left(\mathcal{H}_{2}, \mathcal{Q}_{2}, N_{2}\right)$ and is defined as $\left(\sigma, \mathcal{Q}_{1} \cap_{\mathrm{r}} \mathcal{Q}_{2}, \min \left(N_{1}, N_{2}\right)\right)$, where $\mathcal{Q}_{1} \cap_{\mathfrak{r}} \mathcal{Q}_{2}=\left(\mathcal{F}, \mathcal{B}_{1} \cap \mathcal{B}_{2}, \min \left(N_{1}, N_{2}\right)\right)$ for all $b_{t} \in \mathcal{B}_{1} \cap$ $\mathcal{B}_{2}, u_{g} \in U,\left\langle\left(u_{g}, r_{a_{g t}}\right), y, z\right\rangle \in \sigma\left(b_{t}\right) \Leftrightarrow r_{a_{g t}}=\min \left(r_{a_{g t}}^{1}, r_{a_{g t}}^{2}\right)$, $y=\min \left(s_{\mathcal{C}}\left(u_{g}, r_{a_{g t}}^{1}\right), s_{\mathcal{D}}\left(u_{g}, r_{a_{g t}}^{2}\right)\right) e^{i \min \left(\omega_{\mathcal{C}}\left(u_{g}, r_{a_{g t}}^{1}\right), \omega_{\mathcal{D}}\left(u_{g}, r_{a_{g t}}^{2}\right)\right)}$, $z=\max \left(k_{\mathcal{C}}\left(u_{g}, r_{a_{g t}}^{1}\right), k_{\mathcal{D}}\left(u_{g}, r_{a_{g t}}^{2}\right)\right) e^{i \max \left(\psi_{\mathcal{C}}\left(u_{g}, r_{a_{g t}}^{1}\right), \psi_{\mathcal{D}}\left(u_{g}, r_{a_{g t}}^{2}\right)\right)}$, if
Definition 3.11 For a $\mathrm{CFFNS}_{f} \mathrm{~S}(\mathcal{H}, \mathcal{Q}, N)$, where $\mathcal{Q}=$ $(\mathcal{F}, \mathcal{B}, N)$ is an $\mathrm{NS}_{f} \mathrm{~S}$, the bottom weak complement of $(\mathcal{H}, \mathcal{Q}, N)$ is $\left(\mathcal{H}, \mathcal{Q}^{<}, N\right)$, and the bottom weak CFF complement of $(\mathcal{H}, \mathcal{Q}, N)$ is $\left(\mathcal{H}^{c}, \mathcal{Q}^{<}, N\right)$, where $\mathcal{Q}^{<}=$ $\left(\mathcal{F}^{<}, \mathcal{B}, N\right)$ is the bottom weak complement of $\mathcal{Q}=$ $(\mathcal{F}, \mathcal{B}, N)$ and defined as follows: $\mathcal{F}^{<}\left(u_{g}\right)\left(b_{t}\right)$ (Fatimah et al $\left\{\begin{array}{cl}0, & \text { if } \mathcal{F}\left(u_{g}\right)\left(b_{t}\right)>0, \\ N-1, & \text { if } \mathcal{F}\left(u_{g}\right)\left(b_{t}\right)=0 .\end{array}\right.$

Table 11 Tabular representation of the top weak complement of $\mathrm{CFF}_{5} \mathrm{~S}(\mathcal{H}, \mathcal{Q}, 5)$ in Example 3.1

\begin{tabular}{llll}
\hline$\left(H, \mathcal{Q}^{>}, 5\right)$ & $b_{1}$ & $b_{2}$ & $b_{3}$ \\
\hline$x_{1}$ & $\left\langle 4,\left(0.5 e^{i 0.9 \pi}, 0.6 e^{i 1.2 \pi}\right)\right\rangle$ & $\left\langle 4,\left(0.7 e^{i 1.5 \pi}, 0.3 e^{i 0.5 \pi}\right)\right\rangle$ & $\left\langle 4,\left(0.3 e^{i 0.6 \pi}, 0.8 e^{i 1.6 \pi}\right)\right\rangle$ \\
$x_{2}$ & $\left\langle 4,\left(0.3 e^{i 0.5 \pi}, 0.7 e^{i 1.5 \pi}\right)\right\rangle$ & $\left\langle 4,\left(0.8 e^{i 1.6 \pi}, 0.4 e^{i 0.6 \pi}\right)\right\rangle$ & $\left\langle 4,\left(0.6 e^{i 0.9 \pi}, 0.5 e^{i 1.2 \pi}\right)\right\rangle$ \\
$x_{3}$ & $\left\langle 4,\left(0.7 e^{i 1.4 \pi}, 0.3 e^{i 0.5 \pi}\right)\right\rangle$ & $\left\langle 0,\left(0.9 e^{i 1.8 \pi}, 0.1 e^{i 0.3 \pi}\right)\right\rangle$ & $\left\langle 4,\left(0.1 e^{i 0.2 \pi}, 0.9 e^{i 1.9 \pi}\right)\right\rangle$ \\
$x_{4}$ & $\left\langle 4,\left(0.1 e^{i 0.2 \pi}, 0.9 e^{i 1.8 \pi}\right)\right\rangle$ & $\left\langle 4,\left(0.5 e^{i 0.9 \pi}, 0.5 e^{i 1.0 \pi}\right)\right\rangle$ & $\left\langle 0,\left(0.9 e^{i 1.9 \pi}, 0.2 e^{i 0.3 \pi}\right)\right\rangle$ \\
\hline
\end{tabular}


Table 12 Tabular representation of the top weak CFF complement of the $\mathrm{CFF}_{5 \mathrm{~S}} \mathrm{~S}$ $(\mathcal{H}, \mathcal{Q}, 5)$ in Example 3.1

\begin{tabular}{llll}
\hline$\left(H^{c}, \mathcal{Q}^{>}, 5\right)$ & $b_{1}$ & $b_{2}$ & $b_{3}$ \\
\hline$x_{1}$ & $\left\langle 4,\left(0.6 e^{i 1.2 \pi}, 0.5 e^{i 0.9 \pi}\right)\right\rangle$ & $\left\langle 4,\left(0.3 e^{i 0.5 \pi}, 0.7 e^{i 1.5 \pi}\right)\right\rangle$ & $\left\langle 4,\left(0.8 e^{i 1.6 \pi}, 0.3 e^{i 0.6 \pi}\right)\right\rangle$ \\
$x_{2}$ & $\left\langle 4,\left(0.7 e^{i 1.5 \pi}, 0.3 e^{i 0.5 \pi}\right)\right\rangle$ & $\left\langle 4,\left(0.4 e^{i 0.6 \pi}, 0.8 e^{i 1.6 \pi}\right)\right\rangle$ & $\left\langle 4,\left(0.5 e^{i 1.2 \pi}, 0.6 e^{i 0.9 \pi}\right)\right\rangle$ \\
$x_{3}$ & $\left\langle 4,\left(0.3 e^{i 0.5 \pi}, 0.7 e^{i 1.4 \pi}\right)\right\rangle$ & $\left\langle 0,\left(0.1 e^{i 0.3 \pi}, 0.9 e^{i 1.8 \pi}\right)\right\rangle$ & $\left\langle 4,\left(0.9 e^{i 1.9 \pi}, 0.1 e^{i 0.2 \pi}\right)\right\rangle$ \\
$x_{4}$ & $\left\langle 4,\left(0.9 e^{i 1.8 \pi}, 0.1 e^{i 0.2 \pi}\right)\right\rangle$ & $\left\langle 4,\left(0.5 e^{i 1.0 \pi}, 0.5 e^{i 0.9 \pi}\right)\right\rangle$ & $\left\langle 0,\left(0.2 e^{i 0.3 \pi}, 0.9 e^{i 1.9 \pi}\right)\right\rangle$ \\
\hline
\end{tabular}

Table 13 Tabular representation of the bottom weak complement of $\mathrm{CFF}_{5} \mathrm{~S}_{f} \mathrm{~S}(\mathcal{H}, \mathcal{Q}, 5)$ in Example 3.1

\begin{tabular}{llll}
\hline$\left(\mathcal{H}, \mathcal{Q}^{<}, 5\right)$ & $b_{1}$ & $b_{2}$ & $b_{3}$ \\
\hline$x_{1}$ & $\left\langle 0,\left(0.5 e^{i 0.9 \pi}, 0.6 e^{i 1.2 \pi}\right)\right\rangle$ & $\left\langle 0,\left(0.7 e^{i 1.5 \pi}, 0.3 e^{i 0.5 \pi}\right)\right\rangle$ & $\left\langle 0,\left(0.3 e^{i 0.6 \pi}, 0.8 e^{i 1.6 \pi}\right)\right\rangle$ \\
$x_{2}$ & $\left\langle 0,\left(0.3 e^{i 0.5 \pi}, 0.7 e^{i 1.5 \pi}\right)\right\rangle$ & $\left\langle 0,\left(0.8 e^{i 1.6 \pi}, 0.4 e^{i 0.6 \pi}\right)\right\rangle$ & $\left\langle 0,\left(0.6 e^{i 0.9 \pi}, 0.5 e^{i 1.2 \pi}\right)\right\rangle$ \\
$x_{3}$ & $\left\langle 0,\left(0.7 e^{i 1.4 \pi}, 0.3 e^{i 0.5 \pi}\right)\right\rangle$ & $\left\langle 0,\left(0.9 e^{i 1.8 \pi}, 0.1 e^{i 0.3 \pi}\right)\right\rangle$ & $\left\langle 4,\left(0.1 e^{i 0.2 \pi}, 0.9 e^{i 1.9 \pi}\right)\right\rangle$ \\
$x_{4}$ & $\left\langle 4,\left(0.1 e^{i 0.2 \pi}, 0.9 e^{i 1.8 \pi}\right)\right\rangle$ & $\left\langle 0,\left(0.5 e^{i 0.9 \pi}, 0.5 e^{i 1.0 \pi}\right)\right\rangle$ & $\left\langle 0,\left(0.9 e^{i 1.9 \pi}, 0.2 e^{i 0.3 \pi}\right)\right\rangle$ \\
\hline
\end{tabular}

Table 14 Tabulated form of the bottom weak CFF complement of the $\mathrm{CFF}_{5} \mathrm{~S} S(\mathcal{H}, \mathcal{Q}, 5)$ in Example 3.1

\begin{tabular}{llll}
\hline$\left(\mathcal{H}^{c}, \mathcal{Q}^{<}, 5\right)$ & $b_{1}$ & $b_{2}$ & $b_{3}$ \\
\hline$x_{1}$ & $\left\langle 0,\left(0.6 e^{i 1.2 \pi}, 0.5 e^{i 0.9 \pi}\right)\right\rangle$ & $\left\langle 0,\left(0.3 e^{i 0.5 \pi}, 0.7 e^{i 1.5 \pi}\right)\right\rangle$ & $\left\langle 0,\left(0.8 e^{i 1.6 \pi}, 0.3 e^{i 0.6 \pi}\right)\right\rangle$ \\
$x_{2}$ & $\left\langle 0,\left(0.7 e^{i 1.5 \pi}, 0.3 e^{i 0.5 \pi}\right)\right\rangle$ & $\left\langle 0,\left(0.4 e^{i 0.6 \pi}, 0.8 e^{i 1.6 \pi}\right)\right\rangle$ & $\left\langle 0,\left(0.5 e^{i 1.2 \pi}, 0.6 e^{i 0.9 \pi}\right)\right\rangle$ \\
$x_{3}$ & $\left\langle 0,\left(0.3 e^{i 0.5 \pi}, 0.7 e^{i 1.4 \pi}\right)\right\rangle$ & $\left\langle 0,\left(0.1 e^{i 0.3 \pi}, 0.9 e^{i 1.8 \pi}\right)\right\rangle$ & $\left\langle 4,\left(0.9 e^{i 1.9 \pi}, 0.1 e^{i 0.2 \pi}\right)\right\rangle$ \\
$x_{4}$ & $\left\langle 4,\left(0.9 e^{i 1.8 \pi}, 0.1 e^{i 0.2 \pi}\right)\right\rangle$ & $\left\langle 0,\left(0.5 e^{i 1.0 \pi}, 0.5 e^{i 0.9 \pi}\right)\right\rangle$ & $\left\langle 0,\left(0.2 e^{i 0.3 \pi}, 0.9 e^{i 1.9 \pi}\right)\right\rangle$ \\
\hline
\end{tabular}

$$
\left\langle\left(u_{g}, r_{a_{g t}}^{1}\right), s_{\mathcal{C}}\left(u_{g}, r_{a_{g t}}^{1}\right) e^{i \omega_{\mathcal{C}}\left(u_{g}, r_{a_{g t}}^{1}\right)}, \quad k_{\mathcal{C}}\left(u_{g}, r_{a_{g t}}^{1}\right) e^{i \psi_{\mathcal{C}}\left(u_{g}, r_{a_{g t}}^{1}\right)}\right\rangle \in
$$

$\mathcal{B}_{1}\left(b_{t}\right) \quad$ and $\quad\left\langle\left(u_{g}, r_{a_{g t}}^{2}\right), s_{\mathcal{D}}\left(u_{g}, r_{a_{g t}}^{2}\right) e^{i \omega_{\mathcal{D}}\left(u_{g}, r_{a_{g t}}^{2}\right)}, \quad k_{\mathcal{D}}\right.$ $\left.\left(u_{g}, r_{a_{g t}}^{2}\right) e^{i \psi_{\mathcal{D}}\left(u, r_{a_{t} t}^{2}\right)}\right\rangle \in \mathcal{B}_{2}\left(b_{t}\right), \mathcal{C}$ and $\mathcal{D}$ are CFFSs on $\mathcal{F}_{1}\left(b_{t}\right)$ and $\mathcal{F}_{2}\left(b_{t}\right)$, respectively.

Example 3.6 Let $\left(\mathcal{H}_{1}, \mathcal{Q}_{1}, 5\right)$ be a $\mathrm{CFF}_{5 \mathrm{~S}} \mathrm{~S}$ and $\left(\mathcal{H}_{2}, \mathcal{Q}_{2}, 4\right)$ be a CFF4S $f$ defined by Tables 15 and 16 , respectively, where $\mathcal{Q}_{1}=\left(\mathcal{F}_{1}, \mathcal{B}_{1}, 5\right)$ and $\mathcal{Q}_{2}=$ $\left(\mathcal{H}_{2}, \mathcal{B}_{2}, 4\right)$ are $\mathrm{NS}_{f} \mathrm{Ss}$ over $U$, then their restricted intersection $\left(\mathcal{H}_{1}, \mathcal{Q}_{1}, 5\right) \cap_{\mathfrak{R}}\left(\mathcal{H}_{2}, \mathcal{Q}_{2}, 4\right)=\left(\sigma, \mathcal{Q}_{1} \cap_{\mathfrak{r}} \mathcal{Q}_{2}, 4\right)$ is defined by Table 17 .
Definition 3.13 Let $U$ be a universe of discourse and $\left(\mathcal{H}_{1}, \mathcal{Q}_{1}, N_{1}\right)$ and $\left(\mathcal{H}_{2}, \mathcal{Q}_{2}, N_{2}\right)$ be two CFFNS $_{f}$ Ss over nonempty set $U$, where $\mathcal{Q}_{1}=\left(\mathcal{F}_{1}, \mathcal{B}_{1}, N_{1}\right)$ and $\mathcal{Q}_{2}=$ $\left(\mathcal{F}_{2}, \mathcal{B}_{2}, N_{2}\right)$ are $\mathrm{NS}_{f} \mathrm{Ss}$ on $U$, then their extended intersection is denoted by $\left(\mathcal{H}_{1}, \mathcal{Q}_{1}, N_{1}\right) \cap_{\mathfrak{E}}\left(\mathcal{H}_{2}, \mathcal{Q}_{2}, N_{2}\right)$ and is defined as $\left(\Im, \mathcal{Q}_{1} \cap_{\mathfrak{e}} \mathcal{Q}_{2}, \max \left(N_{1}, N_{2}\right)\right)$, where $\mathcal{Q}_{1} \cap_{\mathfrak{e}} \mathcal{Q}_{2}=$ $\left(\mathcal{H}, \mathcal{B}_{1} \cup \mathcal{B}_{2}, \max \left(N_{1}, N_{2}\right)\right)$, and $\Im\left(b_{t}\right)$ is given by:
Table 15 Tabular representation of CFF5S $\mathrm{S}_{f} \mathrm{~S}\left(\mathcal{H}_{1}, \mathcal{Q}_{1}, 5\right)$ in Example 3.6

\begin{tabular}{llll}
\hline$\left(\mathcal{H}_{1}, \mathcal{Q}_{1}, 5\right)$ & $b_{1}$ & $b_{2}$ & $b_{3}$ \\
\hline$x_{1}$ & $\left\langle 4,\left(0.92 e^{i 1.88 \pi}, 0.08 e^{i 0.36 \pi}\right)\right\rangle$ & $\left\langle 3,\left(0.77 e^{i 1.58 \pi}, 0.37 e^{i 0.69 \pi}\right)\right\rangle$ & $\left\langle 2,\left(0.61 e^{i 1.14 \pi}, 0.42 e^{i 1.29 \pi}\right)\right\rangle$ \\
$x_{2}$ & $\left\langle 3,\left(0.73 e^{i 1.46 \pi}, 0.27 e^{i 0.78 \pi}\right)\right\rangle$ & $\left\langle 4,\left(0.92 e^{i 1.96 \pi}, 0.15 e^{i 0.29 \pi}\right)\right\rangle$ & $\left\langle 1,\left(0.37 e^{i 0.63 \pi}, 0.59 e^{i 1.54 \pi}\right)\right\rangle$ \\
$x_{3}$ & $\left\langle 4,\left(0.89 e^{i 1.76 \pi}, 0.09 e^{i 0.36 \pi}\right)\right\rangle$ & $\left\langle 2,\left(0.49 e^{i 1.27 \pi}, 0.57 e^{i 1.18 \pi}\right)\right\rangle$ & $\left\langle 0,\left(0.05 e^{i 0.32 \pi}, 0.92 e^{i 1.94 \pi}\right)\right\rangle$ \\
$x_{4}$ & $\left\langle 1,\left(0.25 e^{i 0.45 \pi}, 0.75 e^{i 1.65 \pi}\right)\right\rangle$ & $\left\langle 0,\left(0.15 e^{i 0.25 \pi}, 0.95 e^{i 1.85 \pi}\right)\right\rangle$ & $\left\langle 2,\left(0.55 e^{i 0.95 \pi}, 0.61 e^{i 0.85 \pi}\right)\right\rangle$ \\
\hline
\end{tabular}


Table 16 Tabulated form of the $\mathrm{CFF}_{4} \mathrm{~S}_{f} \mathrm{~S}\left(\mathcal{H}_{2}, \mathcal{Q}_{2}, 4\right)$ in Example 3.6

\begin{tabular}{llll}
\hline$\left(\mathcal{H}_{2}, \mathcal{Q}_{2}, 4\right)$ & $b_{1}$ & $b_{2}$ & 0 \\
\hline$x_{1}$ & $\left\langle 0,\left(0.05 e^{i 0.42 \pi}, 0.85 e^{i 1.76 \pi}\right)\right\rangle$ & $\left\langle 2,\left(0.48 e^{i 1.57 \pi}, 0.29 e^{i 0.67 \pi}\right)\right\rangle$ & $\left\langle 3,\left(0.88 e^{i 1.76 \pi}, 0.05 e^{i 0.19 \pi}\right)\right\rangle$ \\
$x_{2}$ & $\left\langle 1,\left(0.33 e^{i 0.57 \pi}, 0.69 e^{i 1.48 \pi}\right)\right\rangle$ & $\left\langle 3,\left(0.97 e^{i 1.96 \pi}, 0.19 e^{i 0.39 \pi}\right)\right\rangle$ & $\left\langle 2,\left(0.69 e^{i 0.97 \pi}, 0.38 e^{i 0.65 \pi}\right)\right\rangle$ \\
$x_{3}$ & $\left\langle 2,\left(0.76 e^{i 1.43 \pi}, 0.39 e^{i 0.66 \pi}\right)\right\rangle$ & $\left\langle 0,\left(0.19 e^{i 0.25 \pi}, 0.89 e^{i 1.94 \pi}\right)\right\rangle$ & $\left\langle 1,\left(0.29 e^{i 0.72 \pi}, 0.58 e^{i 1.33 \pi}\right)\right\rangle$ \\
$x_{4}$ & $\left\langle 3,\left(0.93 e^{i 1.88 \pi}, 0.15 e^{i 0.25 \pi}\right)\right\rangle$ & $\left\langle 1,\left(0.38 e^{i 0.67 \pi}, 0.76 e^{i 1.29 \pi}\right)\right\rangle$ & $\left\langle 0,\left(0.12 e^{i 0.37 \pi}, 0.91 e^{i 1.87 \pi}\right)\right\rangle$ \\
\hline
\end{tabular}

\begin{tabular}{lll}
\hline$\left(\sigma, \mathcal{Q}_{1} \cap_{\mathrm{r}} \mathcal{Q}_{2}, 4\right)$ & $b_{1}$ & $b_{2}$ \\
\hline$x_{1}$ & $\left\langle 0,\left(0.05 e^{i 0.42 \pi}, 0.85 e^{i 1.76 \pi}\right)\right\rangle$ & $\left\langle 2,\left(0.48 e^{i 1.57 \pi}, 0.37 e^{i 0.69 \pi}\right)\right\rangle$ \\
$x_{2}$ & $\left\langle 1,\left(0.33 e^{i 0.57 \pi}, 0.69 e^{i 1.48 \pi}\right)\right\rangle$ & $\left\langle 3,\left(0.92 e^{i 1.96 \pi}, 0.19 e^{i 0.39 \pi}\right)\right\rangle$ \\
$x_{3}$ & $\left\langle 2,\left(0.76 e^{i 1.43 \pi}, 0.39 e^{i 0.66 \pi}\right)\right\rangle$ & $\left\langle 0,\left(0.19 e^{i 0.25 \pi}, 0.89 e^{i 1.94 \pi}\right)\right\rangle$ \\
$x_{4}$ & $\left\langle 1,\left(0.25 e^{i 0.45 \pi}, 0.75 e^{i 1.65 \pi}\right)\right\rangle$ & $\left\langle 0,\left(0.15 e^{i 0.25 \pi}, 0.95 e^{i 1.85 \pi}\right)\right\rangle$ \\
\hline
\end{tabular}

Table 17 Tabulated form of $\left(\mathcal{H}_{1}, \mathcal{Q}_{1}, 5\right) \cap_{\Re}\left(\mathcal{H}_{2}, \mathcal{Q}_{2}, 4\right)$ defined in Example 3.6
$\Im\left(b_{t}\right)=\left\{\begin{array}{c}\mathcal{H}_{1}\left(b_{t}\right), \\ \mathcal{H}_{2}\left(b_{t}\right), \\ \left\langle\left(u_{g}, r_{a_{g l}}\right), y, z\right\rangle, \\ \end{array}\right.$ if $b_{t} \in \mathcal{B}_{1}-\mathcal{B}_{2}$,

if $b_{t} \in \mathcal{B}_{1}-\mathcal{B}_{2}$,

such that $r_{a_{g t}}=\min \left(r_{a_{g t}}^{1}, r_{a_{g t}}^{2}\right)$,

$y=\min \left(s_{\mathcal{C}}\left(u_{g}, r_{a_{g t}}^{1}\right), s_{\mathcal{D}}\left(u_{g}, r_{a_{g t}}^{2}\right)\right) e^{i \min \left(\omega_{\mathcal{C}}\left(u_{g}, r_{a_{g t}}^{1}\right), \omega_{\mathcal{D}}\left(u_{g}, r_{a_{t} t}^{2}\right)\right)}$,

$z=\max \left(k_{\mathcal{C}}\left(u_{g}, r_{a_{g t} t}^{1}\right), k_{\mathcal{D}}\left(u_{g}, r_{a_{g} t}^{2}\right)\right) e^{i \max \left(\psi_{\mathcal{C}}\left(u_{g}, r_{a_{t} t}^{1}\right), \psi_{\mathcal{D}}\left(u_{g}, r_{a_{g} t}^{2}\right)\right)}$,

where $\left\langle\left(u_{g}, r_{a_{g t}}^{1}\right), s_{\mathcal{C}}\left(u_{g}, r_{a_{g t}}^{1}\right) e^{i \omega_{\mathcal{C}}\left(u_{g}, r_{a_{g t}}^{1}\right)}, k_{\mathcal{C}}\left(u_{g}, r_{a_{g t}}^{1}\right) e^{i \psi_{\mathcal{C}}\left(u_{g}, r_{a_{g t}}^{1}\right)}\right\rangle \in \mathcal{B}_{1}\left(b_{t}\right)$

and $\left\langle\left(u_{g}, r_{a_{g t}}^{2}\right), s_{\mathcal{D}}\left(u_{g}, r_{a_{g t}}^{2}\right) e^{i \omega_{\mathcal{D}}\left(u_{g}, r_{a_{g t} t}^{2}\right)}, k_{\mathcal{D}}\left(u_{g}, r_{a_{g t}}^{2}\right) e^{i \psi_{\mathcal{D}}\left(u_{g}, r_{a_{g} t}^{2}\right)}\right\rangle \in \mathcal{B}_{2}\left(b_{t}\right)$,

$\mathcal{C}$ and DareCFFSson $\mathcal{F}_{1}\left(b_{t}\right)$ and $\mathcal{F}_{2}\left(b_{t}\right)$, respectively.
Example 3.7 The extended intersection $\left(\Im, \mathcal{Q}_{1} \cap_{\mathfrak{e}} \mathcal{Q}_{2}, 5\right)$ of $\left(\mathcal{H}_{1}, \mathcal{Q}_{1}, 5\right)$ (see Table 15) and $\left(\mathcal{H}_{2}, \mathcal{Q}_{2}, 4\right)$ (see Table 16) is shown by Table 18.

Definition 3.14 Let $U$ be a universe of discourse and $\left(\mathcal{H}_{1}, \mathcal{Q}_{1}, N_{1}\right)$ and $\left(\mathcal{H}_{2}, \mathcal{Q}_{2}, N_{2}\right)$ be two $\mathrm{CFFNS}_{f}$ Ss over nonempty set $U$, where $\mathcal{Q}_{1}=\left(\mathcal{F}_{1}, \mathcal{B}_{1}, N_{1}\right)$ and $\mathcal{Q}_{2}=$ $\left(\mathcal{F}_{2}, \mathcal{B}_{2}, N_{2}\right)$ are $\mathrm{NS}_{f}$ Ss on $U$, then their restricted union is denoted by $\left(\mathcal{F}_{1}, \mathcal{Q}_{1}, N_{1}\right) \cup_{\mathfrak{R}}\left(\mathcal{F}_{2}, \mathcal{Q}_{2}, N_{2}\right)$ and is defined as $\left(\hbar, \mathcal{Q}_{1} \cup_{\Re} \mathcal{Q}_{2}, \max \left(N_{1}, N_{2}\right)\right)$, where $\mathcal{Q}_{1} \cup_{\Re} \mathcal{Q}_{2}=\left(\mathcal{S}, \mathcal{B}_{1} \cap\right.$ $\left.\mathcal{B}_{2}, \max \left(N_{1}, N_{2}\right)\right)$ for all $b_{t} \in \mathcal{B}_{1} \cap \mathcal{B}_{2}, u_{g} \in U,\left\langle\left(u_{g}, r_{a_{g t}}\right)\right.$, $y, z\rangle \in \hbar\left(b_{t}\right) \Leftrightarrow r_{a_{g t}}=\max \left(r_{a_{g t}}^{1}, r_{a_{g t}}^{2}\right), y=\max \left(s_{\mathcal{C}}\left(u_{g}, r_{a_{g t}}^{1}\right)\right.$, $\left.s_{\mathcal{D}}\left(u_{g}, r_{a_{g t}}^{2}\right)\right) e^{i \max \left(\omega_{\mathcal{C}}\left(u_{g}, r_{a_{g t}}^{1}\right), \omega_{\mathcal{D}}\left(u_{g}, r_{a_{g t}}^{2}\right)\right)}, \quad z=\min \left(k_{\mathcal{C}}\left(u_{g}, r_{a_{g t}}^{1}\right)\right.$, $\left.k_{\mathcal{D}}\left(u_{g}, r_{a_{g t}}^{2}\right)\right) e^{i \min \left(\psi_{\mathcal{C}}\left(u_{g}, r_{a_{g t}}^{1}\right), \psi_{\mathcal{D}}\left(u_{g}, r_{a_{g t}}^{2}\right)\right)}$, if $\left\langle\left(u_{g}, r_{a_{g t}}^{1}\right), s_{\mathcal{C}}\left(u_{g}, r_{a_{g t}}^{1}\right) e^{i \omega_{\mathcal{C}}\left(u_{g}, r_{a_{g t} t}^{1}\right)}, \quad k_{\mathcal{C}}\left(u_{g}, r_{a_{g t}}^{1}\right) e^{i \psi_{\mathcal{C}}\left(u_{g}, r_{a_{g} t}^{1}\right)}\right\rangle \in$ $\mathcal{B}_{1}\left(b_{t}\right) \quad$ and $\left\langle\left(u_{g}, r_{a_{g t}}^{2}\right), s_{\mathcal{D}}\left(u_{g}, r_{a_{g t}}^{2}\right) e^{i \omega_{\mathcal{D}}\left(u_{g}, r_{a_{g t}}^{2}\right)}, \quad k_{\mathcal{D}}\left(u_{g}, r_{a_{g t}}^{2}\right)\right.$

Table 18 Tabulated form of the extended intersection $\left(\mathcal{H}_{1}, \mathcal{Q}_{1}, 5\right) \cap_{\mathfrak{E}}\left(\mathcal{H}_{2}, \mathcal{Q}_{2}, 4\right)$ in Example 3.7

\begin{tabular}{lllll}
\hline$\left(\Im, \mathcal{Q}_{1} \cap_{\mathrm{e}} \mathcal{Q}_{2}, 5\right)$ & $b_{1}$ & $b_{2}$ & 0 \\
\hline$x_{1}$ & $\left\langle 0,\left(0.05 e^{i 0.42 \pi}, 0.85 e^{i 1.76 \pi}\right)\right\rangle$ & $\left\langle 2,\left(0.48 e^{i 1.57 \pi}, 0.37 e^{i 0.69 \pi}\right)\right\rangle$ & $\left\langle 2,\left(0.61 e^{i 1.14 \pi}, 0.42 e^{i 1.29 \pi}\right)\right\rangle$ & $\left\langle 3,\left(0.88 e^{i 1.76 \pi}, 0.05 e^{i 0.19 \pi}\right)\right\rangle$ \\
$x_{2}$ & $\left\langle 1,\left(0.33 e^{i 0.57 \pi}, 0.69 e^{i 1.48 \pi}\right)\right\rangle$ & $\left\langle 3,\left(0.92 e^{i 1.96 \pi}, 0.19 e^{i 0.39 \pi}\right)\right\rangle$ & $\left\langle 1,\left(0.37 e^{i 0.63 \pi}, 0.59 e^{i 1.54 \pi}\right)\right\rangle$ & $\left\langle 2,\left(0.69 e^{i 0.97 \pi}, 0.38 e^{i 0.65 \pi}\right)\right\rangle$ \\
$x_{3}$ & $\left\langle 2,\left(0.76 e^{i 1.43 \pi}, 0.39 e^{i 0.66 \pi}\right)\right\rangle$ & $\left\langle 0,\left(0.19 e^{i 0.25 \pi}, 0.89 e^{i 1.94 \pi}\right)\right\rangle$ & $\left\langle 0,\left(0.05 e^{i 0.32 \pi}, 0.92 e^{i 1.94 \pi}\right)\right\rangle$ & $\left\langle 1,\left(0.29 e^{i 0.72 \pi}, 0.58 e^{i 1.33 \pi}\right)\right\rangle$ \\
$x_{4}$ & $\left\langle 1,\left(0.25 e^{i 0.45 \pi}, 0.75 e^{i 1.65 \pi}\right)\right\rangle$ & $\left\langle 0,\left(0.15 e^{i 0.25 \pi}, 0.95 e^{i 1.85 \pi}\right)\right\rangle$ & $\left\langle 2,\left(0.55 e^{i 0.95 \pi}, 0.61 e^{i 0.85 \pi}\right)\right\rangle$ & $\left\langle 0,\left(0.12 e^{i 0.37 \pi}, 0.91 e^{i 1.87 \pi}\right)\right\rangle$ \\
\hline
\end{tabular}


$\left.e^{i \psi_{\mathcal{D}}\left(u_{g}, r_{a_{g t}}^{2}\right)}\right\rangle \in \mathcal{B}_{2}\left(b_{t}\right), \mathcal{C}$ and $\mathcal{D}$ are CFFSs on $\mathcal{F}_{1}\left(b_{t}\right)$ and $\mathcal{F}_{2}\left(b_{t}\right)$, respectively.

Example 3.8 The restricted union $\left(\hbar, \mathcal{Q}_{1} \cup_{\Re} \mathcal{Q}_{2}, 5\right)$ of $\left(\mathcal{H}_{1}, \mathcal{Q}_{1}, 5\right)$ (see Table 15) and $\left(\mathcal{H}_{2}, \mathcal{Q}_{2}, 4\right)$ (see Table 16 ) is defined by Table 19 .
Definition 3.16 Suppose that $U$ be a universe of discourse and $(\mathcal{H}, \mathcal{Q}, N)$ be a $\mathrm{CFFNS}_{f} \mathrm{~S}$ over non-empty set $U$, where $\mathcal{Q}=(\mathcal{F}, \mathcal{B}, N)$ is an $\mathrm{NS}_{f} \mathrm{~S}$ on $U$. Let $0<L<N$ be a threshold. A $\mathrm{CFFS}_{f} \mathrm{~S}$ related with $(\mathcal{H}, \mathcal{Q}, N)$ and $L$, denoted by $\left(\mathcal{H}^{L}, \mathcal{B}\right)$, is given as follows:

$\mathcal{H}^{L}\left(b_{t}\right)=\left\{\begin{array}{cc}\left(s\left(u_{g}, r_{a_{g t}}\right) e^{i \omega\left(u_{g}, r_{a_{t} t}\right)}, k\left(u_{g}, r_{a_{g t}}\right) e^{i \psi\left(u_{g}, r_{a_{g} t}\right)}\right), & \text { if } \mathcal{H}\left(b_{t}\right)=\left\langle\left(u_{g}, r_{a_{g t} t}\right), s\left(u_{g}, r_{a_{g t}}\right) e^{i \omega\left(u_{g}, r_{a_{g} t}\right)}, k\left(u_{g}, r_{a_{g t}}\right)\right. \\ \left.e^{i \psi\left(u_{g}, r_{a_{g t}}\right)}\right\rangle, & \text { and } r_{a_{g t}} \geq L, \\ \left(0.0 e^{i 0.0 \pi}, 1.0 e^{i 2.0 \pi}\right) & \text { otherwise. }\end{array}\right.$

Definition 3.15 Let $U$ be a universe of discourse and $\left(\mathcal{H}_{1}, \mathcal{Q}_{1}, N_{1}\right)$ and $\left(\mathcal{H}_{2}, \mathcal{Q}_{2}, N_{2}\right)$ be two CFFNS $_{f}$ Ss over nonempty set $U$, where $\mathcal{Q}_{1}=\left(\mathcal{F}_{1}, \mathcal{B}_{1}, N_{1}\right)$ and $\mathcal{Q}_{2}=$ $\left(\mathcal{F}_{2}, \mathcal{B}_{2}, N_{2}\right)$ are $\mathrm{NS}_{f} \mathrm{Ss}$ on $U$, then their extended union is denoted by $\left(\mathcal{H}_{1}, \mathcal{Q}_{1}, N_{1}\right) \cup \mathfrak{E}\left(\mathcal{H}_{2}, \mathcal{Q}_{2}, N_{2}\right)$ and is defined as $\left(\zeta, \mathcal{Q}_{1} \cup_{\epsilon} \mathcal{Q}_{2}, \max \left(N_{1}, N_{2}\right)\right)$, where $\quad \mathcal{Q}_{1} \cup_{\epsilon} \mathcal{Q}_{2}=$ $\left(\mathfrak{Y}, \mathcal{B}_{1} \cup \mathcal{B}_{2}, \max \left(N_{1}, N_{2}\right)\right)$, and $\zeta\left(b_{t}\right)$ is given by:
Particularly, $\left(\mathcal{H}^{1}, \mathcal{B}\right)$ is called the bottom $\mathrm{CFFS}_{f} \mathrm{~S}$ linked with $(\mathcal{H}, \mathcal{Q}, N)$ and $\left(\mathcal{H}^{N-1}, \mathcal{B}\right)$ is called top $\mathrm{CFFS}_{f} \mathrm{~S}$ associated with $(\mathcal{H}, \mathcal{Q}, N)$.

Definition 3.17 Let $0<L<N$ and $\partial \in[0,1]$ be two thresholds. The $\mathrm{S}_{f} \mathrm{~S}$ over $U$ associated with $(\mathcal{H}, \mathcal{Q}, N)$ and $(L, \partial)$ is $\left(\tilde{\mathfrak{f}}^{(L, \partial)}, \mathcal{B}\right)$ given by: for each $b_{t} \in \mathcal{B}, \tilde{f}^{(L, \partial)}\left(b_{t}\right)=\left\{u_{g} \in U: \mathcal{H}^{L}\left(b_{t}\right)>\partial\right\}$.

$$
\zeta\left(b_{t}\right)=\left\{\begin{array}{cl}
\mathcal{H}_{1}\left(b_{t}\right), & \text { if } b_{t} \in \mathcal{B}_{1}-\mathcal{B}_{2}, \\
\mathcal{H}_{2}\left(b_{t}\right), & \text { if } b_{t} \in \mathcal{B}_{2}-\mathcal{B}_{1}, \\
\left\langle\left(u_{g}, r_{a_{g t}}\right), y, z\right\rangle, & \text { such that } r_{a_{g t}}=\max \left(r_{a_{g t}}^{1}, r_{a_{g t}}^{2}\right), \\
& y=\max \left(s_{\mathcal{C}}\left(u_{g}, r_{a_{g t}}^{1}\right), s_{\mathcal{D}}\left(u_{g}, r_{a_{g t}}^{2}\right)\right) e^{i \max \left(\omega_{\mathcal{C}}\left(u_{g}, r_{a_{g t}}^{1}\right), \omega_{\mathcal{D}}\left(u_{g}, r_{a_{g} t}^{2}\right)\right)}, \\
& z=\min \left(k_{\mathcal{C}}\left(u_{g}, r_{a_{g t}}^{1}\right), k_{\mathcal{D}}\left(u_{g}, r_{a_{g t}}^{2}\right)\right) e^{i \min \left(\psi_{\mathcal{C}}\left(u_{g}, r_{a_{g t}}^{1}\right), \psi_{\mathcal{D}}\left(u_{g}, r_{a_{g t}}^{2}\right)\right)}, \\
& \text { where }\left\langle\left(u_{g}, r_{a_{g t}}^{1}\right), s_{\mathcal{C}}\left(u_{g}, r_{a_{g t}}^{1}\right) e^{i \omega_{\mathcal{C}}\left(u_{g}, r_{a_{g t}}^{1}\right)}, k_{\mathcal{C}}\left(u_{g}, r_{a_{g t}}^{1}\right) e^{i \psi_{\mathcal{C}}\left(u_{g}, r_{a_{g t}}^{1}\right)}\right\rangle \in \mathcal{B}_{1}\left(b_{t}\right) \\
& \text { and }\left\langle\left(u_{g}, r_{a_{g t}}^{2}\right), s_{\mathcal{D}}\left(u_{g}, r_{a_{g t}}^{2}\right) e^{i \omega_{\mathcal{D}}\left(u_{g}, r_{a_{g} t}^{2}\right)}, k_{\mathcal{D}}\left(u_{g}, r_{a_{g t}}^{2}\right) e^{i \psi_{\mathcal{D}}\left(u_{g}, r_{a_{g t} t}^{2}\right)}\right\rangle \in \mathcal{B}_{2}\left(b_{t}\right), \\
& \mathcal{C} \text { and } \mathcal{D} \text { are CFFSs on } \mathcal{F}_{1}\left(b_{t}\right) \text { and } \mathcal{F}_{2}\left(b_{t}\right), \text { respectively. }
\end{array}\right.
$$

Example 3.9 The extended union $\left(\zeta, \mathcal{Q}_{1} \cup_{\epsilon} \mathcal{Q}_{2}, 5\right)$ of $\left(\mathcal{H}_{1}, \mathcal{Q}_{1}, 5\right)$ (see Table 15) and $\left(\mathcal{H}_{2}, \mathcal{Q}_{2}, 4\right)$ (see Table 16) is represented by Table 20 .
Example 3.10 Consider the $\mathrm{CFF}_{5} \mathrm{~S}_{f} \mathrm{~S}$ in Example 3.1, represented by Table 6. From Definition 3.16, we can find the associated $\mathrm{CFFS}_{f} \mathrm{Ss}$ with $\mathrm{CFF}_{5} \mathrm{~S}_{f} \mathrm{~S}$. Let $0<L<5$ be threshold. Then the possible $\mathrm{CFFS}_{f} \mathrm{~S}$ associated with thresholds 1, 2, 3 and 4 are shown by Tables 21-24.
Table 19 Tabulated form of $\left(\mathcal{H}_{1}, \mathcal{Q}_{1}, 5\right) \cup_{\mathfrak{R}}\left(\mathcal{H}_{2}, \mathcal{Q}_{2}, 4\right)$ in Example 3.8

\begin{tabular}{lll}
\hline$\left(\hbar, \mathcal{Q}_{1} \cup_{\Re} \mathcal{Q}_{2}, 5\right)$ & $b_{1}$ & $b_{2}$ \\
\hline$x_{1}$ & $\left\langle 4,\left(0.92 e^{i 1.88 \pi}, 0.08 e^{i 0.36 \pi}\right)\right\rangle$ & $\left\langle 3,\left(0.77 e^{i 1.58 \pi}, 0.29 e^{i 0.67 \pi}\right)\right\rangle$ \\
$x_{2}$ & $\left\langle 3,\left(0.73 e^{i 1.46 \pi}, 0.27 e^{i 0.78 \pi}\right)\right\rangle$ & $\left\langle 4,\left(0.97 e^{i 1.96 \pi}, 0.15 e^{i 0.29 \pi}\right)\right\rangle$ \\
$x_{3}$ & $\left\langle 4,\left(0.89 e^{i 1.76 \pi}, 0.09 e^{i 0.36 \pi}\right)\right\rangle$ & $\left\langle 2,\left(0.49 e^{i 1.27 \pi}, 0.57 e^{i 1.18 \pi}\right)\right\rangle$ \\
$x_{4}$ & $\left\langle 3,\left(0.93 e^{i 1.88 \pi}, 0.15 e^{i 0.25 \pi}\right)\right\rangle$ & $\left\langle 1,\left(0.38 e^{i 0.67 \pi}, 0.76 e^{i 1.29 \pi}\right)\right\rangle$ \\
\hline
\end{tabular}


Table 20 Tabulated form of $\left(\mathcal{H}_{1}, \mathcal{Q}_{1}, 5\right) \cup_{\mathfrak{E}}\left(\mathcal{H}_{2}, \mathcal{Q}_{2}, 4\right)$ given in Example 3.9

\begin{tabular}{lllll}
\hline$\left(\zeta, \mathcal{Q}_{1} \cup_{\epsilon} \mathcal{Q}_{2}, 5\right)$ & $b_{1}$ & $b_{2}$ & $b_{3}$ & 0 \\
\hline$x_{1}$ & $\left\langle 4,\left(0.92 e^{i 1.88 \pi}, 0.08 e^{i 0.36 \pi}\right)\right\rangle$ & $\left\langle 3,\left(0.77 e^{i 1.58 \pi}, 0.29 e^{i 0.67 \pi}\right)\right\rangle$ & $\left\langle 2,\left(0.61 e^{i 1.14 \pi}, 0.42 e^{i 1.29 \pi}\right)\right\rangle$ & $\left\langle 3,\left(0.88 e^{i 1.76 \pi}, 0.05 e^{i 0.19 \pi}\right)\right\rangle$ \\
$x_{2}$ & $\left\langle 3,\left(0.73 e^{i 1.46 \pi}, 0.27 e^{i 0.78 \pi}\right)\right\rangle$ & $\left\langle 4,\left(0.97 e^{i 1.96 \pi}, 0.15 e^{i 0.29 \pi}\right)\right\rangle$ & $\left\langle 1,\left(0.37 e^{i 0.63 \pi}, 0.59 e^{i 1.54 \pi}\right)\right\rangle$ & $\left\langle 2,\left(0.69 e^{i 0.97 \pi}, 0.38 e^{i 0.65 \pi}\right)\right\rangle$ \\
$x_{3}$ & $\left\langle 4,\left(0.89 e^{i 1.76 \pi}, 0.09 e^{i 0.36 \pi}\right)\right\rangle$ & $\left\langle 2,\left(0.49 e^{i 1.27 \pi}, 0.57 e^{i 1.18 \pi}\right)\right\rangle$ & $\left\langle 0,\left(0.05 e^{i 0.32 \pi}, 0.92 e^{i 1.94 \pi}\right)\right\rangle$ & $\left\langle 1,\left(0.29 e^{i 0.72 \pi}, 0.58 e^{i 1.33 \pi}\right)\right\rangle$ \\
$x_{4}$ & $\left\langle 3,\left(0.93 e^{i 1.88 \pi}, 0.15 e^{i 0.25 \pi}\right)\right\rangle$ & $\left\langle 1,\left(0.38 e^{i 0.67 \pi}, 0.76 e^{i 1.29 \pi}\right)\right\rangle$ & $\left\langle 2,\left(0.55 e^{i 0.95 \pi}, 0.61 e^{i 0.85 \pi}\right)\right\rangle$ & $\left\langle 0,\left(0.12 e^{i 0.37 \pi}, 0.91 e^{i 1.87 \pi}\right)\right\rangle$ \\
\hline
\end{tabular}

Table $21 \mathrm{CFFS}_{f} \mathrm{~S}$ associated with $\mathrm{CFF}_{5} \mathrm{~S}_{f} \mathrm{~S}$ and threshold 1

Table $22 \mathrm{CFFS}_{f} \mathrm{~S}$ associated with $\mathrm{CFF}_{5} \mathrm{~S}_{f} \mathrm{~S}$ and threshold 2

Table $23 \mathrm{CFFS}_{f} \mathrm{~S}$ associated with $\mathrm{CFF}_{5 \mathrm{~S}} \mathrm{~S}$ and threshold 3

Table $24 \mathrm{CFFS}_{f} \mathrm{~S}$ associated with $\mathrm{CFF}_{5} \mathrm{~S}_{f} \mathrm{~S}$ and threshold 4

\begin{tabular}{llll}
\hline$\left(\mathcal{H}^{1}, \mathcal{B}\right)$ & $b_{1}$ & $b_{2}$ & $b_{3}$ \\
\hline$x_{1}$ & $\left(0.5 e^{i 0.9 \pi}, 0.6 e^{i 1.2 \pi}\right)$ & $\left(0.7 e^{i 1.5 \pi}, 0.3 e^{i 0.5 \pi}\right)$ & $\left(0.3 e^{i 0.6 \pi}, 0.8 e^{i 1.6 \pi}\right)$ \\
$x_{2}$ & $\left(0.3 e^{i 0.5 \pi}, 0.7 e^{i 1.5 \pi}\right)$ & $\left(0.8 e^{i 1.6 \pi}, 0.4 e^{i 0.6 \pi}\right)$ & $\left(0.6 e^{i 0.9 \pi}, 0.5 e^{i 1.2 \pi}\right)$ \\
$x_{3}$ & $\left(0.7 e^{i 1.4 \pi}, 0.3 e^{i 0.5 \pi}\right)$ & $\left(0.9 e^{i 1.8 \pi}, 0.1 e^{i 0.3 \pi}\right)$ & $\left(0.0 e^{i 0.0 \pi}, 1.0 e^{i 2.0 \pi}\right)$ \\
$x_{4}$ & $\left(0.0 e^{i 0.0 \pi}, 1.0 e^{i 2.0 \pi}\right)$ & $\left(0.5 e^{i 0.9 \pi}, 0.5 e^{i 1.0 \pi}\right)$ & $\left(0.9 e^{i 1.9 \pi}, 0.2 e^{i 0.3 \pi}\right)$ \\
\hline
\end{tabular}

\begin{tabular}{llll}
\hline$\left(\mathcal{H}^{2}, \mathcal{B}\right)$ & $b_{1}$ & $b_{2}$ & $b_{3}$ \\
\hline$x_{1}$ & $\left(0.5 e^{i 0.9 \pi}, 0.6 e^{i 1.2 \pi}\right)$ & $\left(0.7 e^{i 1.5 \pi}, 0.3 e^{i 0.5 \pi}\right)$ & $\left(0.0 e^{i 0.0 \pi}, 1.0 e^{i 2.0 \pi}\right)$ \\
$x_{2}$ & $\left(0.0 e^{i 0.0 \pi}, 1.0 e^{i 2.0 \pi}\right)$ & $\left(0.8 e^{i 1.6 \pi}, 0.4 e^{i 0.6 \pi}\right)$ & $\left(0.6 e^{i 0.9 \pi}, 0.5 e^{i 1.2 \pi}\right)$ \\
$x_{3}$ & $\left(0.7 e^{i 1.4 \pi}, 0.3 e^{i 0.5 \pi}\right)$ & $\left(0.9 e^{i 1.8 \pi}, 0.1 e^{i 0.3 \pi}\right)$ & $\left(0.0 e^{i 0.0 \pi}, 1.0 e^{i 2.0 \pi}\right)$ \\
$x_{4}$ & $\left(0.0 e^{i 0.0 \pi}, 1.0 e^{i 2.0 \pi}\right)$ & $\left(0.5 e^{i 0.9 \pi}, 0.5 e^{i 1.0 \pi}\right)$ & $\left(0.9 e^{i 1.9 \pi}, 0.2 e^{i 0.3 \pi}\right)$ \\
\hline
\end{tabular}

\begin{tabular}{llll}
\hline$\left(\mathcal{H}^{3}, \mathcal{B}\right)$ & $b_{1}$ & $b_{2}$ & $b_{3}$ \\
\hline$x_{1}$ & $\left(0.0 e^{i 0.0 \pi}, 1.0 e^{i 2.0 \pi}\right)$ & $\left(0.7 e^{i 1.5 \pi}, 0.3 e^{i 0.5 \pi}\right)$ & $\left(0.0 e^{i 0.0 \pi}, 1.0 e^{i 2.0 \pi}\right)$ \\
$x_{2}$ & $\left(0.0 e^{i 0.0 \pi}, 1.0 e^{i 2.0 \pi}\right)$ & $\left(0.8 e^{i 1.6 \pi}, 0.4 e^{i 0.6 \pi}\right)$ & $\left(0.0 e^{i 0.0 \pi}, 1.0 e^{i 2.0 \pi}\right)$ \\
$x_{3}$ & $\left(0.7 e^{i 1.4 \pi}, 0.3 e^{i 0.5 \pi}\right)$ & $\left(0.9 e^{i 1.8 \pi}, 0.1 e^{i 0.3 \pi}\right)$ & $\left(0.0 e^{i 0.0 \pi}, 1.0 e^{i 2.0 \pi}\right)$ \\
$x_{4}$ & $\left(0.0 e^{i 0.0 \pi}, 1.0 e^{i 2.0 \pi}\right)$ & $\left(0.0 e^{i 0.0 \pi}, 1.0 e^{i 2.0 \pi}\right)$ & $\left(0.9 e^{i 1.9 \pi}, 0.2 e^{i 0.3 \pi}\right)$ \\
\hline
\end{tabular}

\begin{tabular}{llll}
\hline$\left(\mathcal{H}^{4}, \mathcal{B}\right)$ & $b_{1}$ & $b_{2}$ & $b_{3}$ \\
\hline$x_{1}$ & $\left(0.0 e^{i 0.0 \pi}, 1.0 e^{i 2.0 \pi}\right)$ & $\left(0.0 e^{i 0.0 \pi}, 1.0 e^{i 2.0 \pi}\right)$ & $\left(0.0 e^{i 0.0 \pi}, 1.0 e^{i 2.0 \pi}\right)$ \\
$x_{2}$ & $\left(0.0 e^{i 0.0 \pi}, 1.0 e^{i 2.0 \pi}\right)$ & $\left(0.0 e^{i 0.0 \pi}, 1.0 e^{i 2.0 \pi}\right)$ & $\left(0.0 e^{i 0.0 \pi}, 1.0 e^{i 2.0 \pi}\right)$ \\
$x_{3}$ & $\left(0.0 e^{i 0.0 \pi}, 1.0 e^{i 2.0 \pi}\right)$ & $\left(0.9 e^{i 1.8 \pi}, 0.1 e^{i 0.3 \pi}\right)$ & $\left(0.0 e^{i 0.0 \pi}, 1.0 e^{i 2.0 \pi}\right)$ \\
$x_{4}$ & $\left(0.0 e^{i 0.0 \pi}, 1.0 e^{i 2.0 \pi}\right)$ & $\left(0.0 e^{i 0.0 \pi}, 1.0 e^{i 2.0 \pi}\right)$ & $\left(0.9 e^{i 1.9 \pi}, 0.2 e^{i 0.3 \pi}\right)$ \\
\hline
\end{tabular}

\section{Operations}

Definition 4.1 Let $\alpha_{a_{1 t}}=\left\langle r_{a_{1 t}},\left(s_{a_{1 t}} e^{i \omega_{a_{1 t}}}, k_{a_{1 t}} e^{i \psi_{a_{1 t}}}\right)\right\rangle(t=$ $1,2)$ and $\alpha=\left\langle r,\left(s e^{i \omega}, k e^{i \psi}\right)\right\rangle$ be three $\operatorname{CFFNS}_{f} \mathrm{Ns}_{\text {over }} U$ and $\xi>0$. Then, some operations for $\mathrm{CFFNS}_{f} \mathrm{Ns}$ are:

1. $\alpha_{a_{11}} \cup \alpha_{a_{12}}=\left\langle\max \left(r_{a_{11}}, r_{a_{12}}\right),\left(\max \left(s_{a_{11}}, s_{a_{12}}\right) e^{i \max \left(\omega_{a_{11}},\right.}\right.\right.$ $\left.\left.\left.\omega_{a_{12}}\right), \min \left(k_{a_{11}}, k_{a_{12}}\right) e^{i \min \left(\psi_{a_{11}}, \psi_{a_{12}}\right)}\right)\right\rangle$
2. $\alpha_{a_{11}} \cap \alpha_{a_{12}}=\left\langle\min \left(r_{a_{11}}, r_{a_{12}}\right),\left(\min \left(s_{a_{11}}, s_{a_{12}}\right)\right.\right.$ $\left.\left.e^{i \min \left(\omega_{a_{11}}, \omega_{a_{12}}\right)}, \max \left(k_{a_{11}}, k_{a_{12}}\right) e^{i \max \left(\psi_{a_{11}}, \psi_{a_{12}}\right)}\right)\right\rangle$

3. $\alpha^{c}=\left\langle r,\left(k e^{i \psi}, s e^{i \omega}\right)\right\rangle$

4. $\alpha_{a_{11}} \oplus \alpha_{a_{12}}=\left\langle\max \left(r_{a_{11}}, r_{a_{12}}\right)\right.$, $\left(\sqrt[3]{\left(s_{a_{11}}\right)^{3}+\left(s_{a_{12}}\right)^{3}-\left(s_{a_{11}}\right)^{3}\left(s_{a_{12}}\right)^{3}}\right.$ $\left.\left.e^{i 2 \pi \sqrt[3]{\left(\frac{\omega_{a_{11}}}{2 \pi}\right)^{3}+\left(\frac{\omega_{a_{12}}}{2 \pi}\right)^{3}-\left(\frac{\omega_{a_{11}}}{2 \pi}\right)^{3}\left(\frac{\omega_{a_{12}}}{2 \pi}\right)^{3}}}, k_{a_{11}} k_{a_{12}} e^{i 2 \pi\left(\frac{\psi a_{11}}{2 \pi}\right)\left(\frac{\psi a_{12}}{2 \pi}\right)}\right)\right\rangle$ 
5. $\quad \alpha_{a_{11}} \otimes \alpha_{a_{12}}=\left\langle\min \left(r_{a_{11}}, r_{a_{12}}\right),\left(s_{a_{11}} s_{a_{12}} e^{i 2 \pi\left(\frac{\omega a_{11}}{2 \pi}\right)\left(\frac{\omega a_{12}}{2 \pi}\right)}\right.\right.$, $\sqrt[3]{\left(k_{a_{11}}\right)^{3}+\left(k_{a_{12}}\right)^{3}-\left(k_{a_{11}}\right)^{3}\left(k_{a_{12}}\right)^{3}}$ $e^{\left.\left.i 2 \pi \sqrt[3]{\left(\frac{\psi_{a_{11}}}{2 \pi}\right)^{3}+\left(\frac{\psi_{a_{12}}}{2 \pi}\right)^{3}-\left(\frac{\psi_{a_{11}}}{2 \pi}\right)^{3}\left(\frac{\psi_{a_{12}}}{2 \pi}\right)^{3}}\right)\right\rangle}$

6. $\xi \alpha=\left\langle r,\left(\sqrt[3]{1-\left(1-s^{3}\right)^{\xi}} e^{i 2 \pi} \sqrt[3]{1-\left(1-\left(\frac{\omega}{2 \pi}\right)^{3}\right)^{\xi}}, k^{\xi} e^{i 2 \pi\left(\frac{\psi}{2 \pi}\right)^{\xi}}\right)\right\rangle$

7. $\alpha^{\xi}=\left\langle r,\left(s^{\xi} e^{i 2 \pi\left(\frac{\omega}{2 \pi}\right)^{\xi}}, \sqrt[3]{1-\left(1-k^{3}\right)^{\xi}} e^{i 2 \pi} \sqrt[3]{1-\left(1-\left(\frac{\psi}{2 \pi}\right)^{3}\right)^{\xi}}\right)\right\rangle$

Definition 4.2 Let $\alpha_{a_{1 t}}=\left\langle r_{a_{1 t}},\left(s_{a_{1 t}} e^{i \omega_{a_{1 t}}}, k_{a_{1 t}} e^{i \psi_{a_{1 t}}}\right)\right\rangle(t=$ $1,2)$ and $\alpha=\left\langle r,\left(s e^{i \omega}, k e^{i l}\right)\right\rangle$ be three $\mathrm{CFFNS}_{f} \mathrm{Ns}$ over $U$ and $\xi>0$. Then, Yager operations for $\mathrm{CFFNS}_{f} \mathrm{Ns}$ are:

1. $\alpha_{a_{11}} \oplus \alpha_{a_{12}}=\left\langle\max \left(r_{a_{11}}, r_{a_{12}}\right),\left(\sqrt[3]{\min \left(1,\left(s_{a_{11}}^{3 \varphi}+s_{a_{12}}^{3 \varphi}\right)^{\frac{1}{\varphi}}\right)}\right.\right.$

$$
\begin{aligned}
& e^{i 2 \pi} \sqrt[3]{\min \left(1,\left(\left(\frac{\omega a_{11}}{2 \pi}\right)^{3 \varphi}+\left(\frac{\omega a_{12}}{2 \pi}\right)^{3 \varphi}\right)^{\frac{1}{\varphi}}\right)}, \\
& \quad \sqrt[3]{1-\min \left(1,\left(\left(1-k_{a_{11}}^{3}\right)^{\varphi}+\left(1-k_{a_{12}}^{3}\right)^{\varphi}\right)^{\frac{1}{\varphi}}\right)}
\end{aligned}
$$

$$
\begin{aligned}
& \alpha_{1} \oplus \alpha_{2}=\left\langle\max (3,2),\left(\sqrt[3]{\min \left(1,\left(0.8^{12}+0.6^{12}\right)^{\frac{1}{4}}\right)} e^{i 2 \pi} \sqrt[3]{\min \left(1,\left(\left(\frac{1.6 \pi}{2 \pi}\right)^{12}+\left(\frac{0.9 \pi}{2 \pi}\right)^{12}\right)^{\frac{1}{4}}\right)},\right.\right. \\
& \left.\left.\left.\sqrt[3]{1-\min \left(1,\left(\left(1-0.4^{3}\right)^{4}+\left(1-0.5^{3}\right)^{4}\right)^{\frac{1}{4}}\right)} e^{i 2 \pi} \sqrt[3]{1-\min \left(1,\left(\left(1-\left(\frac{0.6 \pi}{2 \pi}\right)^{3}\right)^{4}+\left(1-\left(\frac{1.2 \pi}{2 \pi}\right)^{3}\right)^{4}\right)^{\frac{1}{4}}\right.}\right)\right)\right\rangle \\
& =\left\langle 3,\left(0.8 e^{i 1.6 \pi}, 0 e^{i 0}\right)\right\rangle \\
& \alpha_{1} \otimes \alpha_{2}=\left\langle\min (3,2),\left(\sqrt[3]{1-\min \left(1,\left(\left(1-0.8^{3}\right)^{4}+\left(1-0.6^{3}\right)^{4}\right)^{\frac{1}{4}}\right.} e^{i 2 \pi} \sqrt[3]{1-\min \left(1,\left(\left(1-\left(\frac{1.6 \pi}{2 \pi}\right)^{3}\right)^{4}+\left(1-\left(\frac{0.9 \pi}{2 \pi}\right)^{3}\right)^{4}\right)^{\frac{1}{4}}\right)}\right.\right.
\end{aligned}
$$

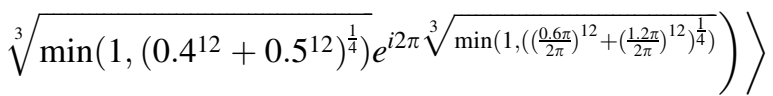

$$
\begin{aligned}
& =\left\langle 2,\left(0.57 e^{i 0.83 \pi}, 0.5 e^{i 1.2 \pi}\right)\right\rangle \\
& 2 \alpha_{1}=\left\langle 3,\left(\sqrt[3]{\min \left(1,\left(2(0.8)^{12}\right)^{\frac{1}{4}}\right)} e^{i 2 \pi \sqrt[3]{\min \left(1,\left(2\left(\frac{1.6 \pi}{2 \pi}\right)^{12}\right)^{\frac{1}{4}}\right.}}, \sqrt[3]{1-\min \left(1,\left(2\left(1-0.4^{3}\right)^{4}\right)^{\frac{1}{4}}\right)}\right.\right. \\
& e^{\left.\left.i 2 \pi \sqrt[3]{1-\min \left(1,\left(2\left(1-\left(\frac{0.6 \pi}{2 \pi}\right)^{3}\right)^{4}\right)^{\frac{1}{4}}\right)}\right)\right\rangle} \\
& =\left\langle 3,\left(0.85 e^{i 1.7 \pi}, 0 e^{i 0}\right)\right\rangle \\
& \left.\alpha_{1}^{2}=\left\langle 3,\left(\sqrt[3]{1-\min \left(1,\left(2\left(1-0.8^{3}\right)^{4}\right)^{\frac{1}{4}}\right.}\right) e^{i 2 \pi} \sqrt[3]{1-\min \left(1,\left(2\left(1-\left(\frac{1.6 \pi}{2 \pi}\right)^{3}\right)^{4}\right)^{\frac{1}{4}}\right.}\right), \sqrt[3]{\min \left(1,\left(2(0.4)^{12}\right)^{\frac{1}{4}}\right.}\right) \\
& e^{\left.\left.i 2 \pi \sqrt[3]{\min \left(1,\left(2\left(\frac{0.6 \pi}{2 \pi}\right)^{12}\right)^{\frac{1}{4}}\right)}\right)\right\rangle} \\
& =\left\langle 3,\left(0.75 e^{i 1.5 \pi}, 0.42 e^{i 0.64 \pi}\right)\right\rangle
\end{aligned}
$$

$$
e^{\left.\left.i 2 \pi \sqrt[3]{1-\min \left(1,\left(\left(1-\left(\frac{\psi_{a 1}}{2 \pi}\right)^{3}\right)^{\varphi}+\left(1-\left(\frac{\psi_{a_{12}}}{2 \pi}\right)^{3}\right)^{\varphi}\right)^{\frac{1}{\varphi}}\right)}\right)\right\rangle}
$$

2. $\alpha_{a_{11}} \otimes \alpha_{a_{12}}=\left\langle\min \left(r_{a_{11}}, r_{a_{12}}\right),\left(\sqrt[3]{1-\min \left(1,\left(\left(1-s_{a_{11}}^{3}\right)^{\varphi}+\left(1-s_{a_{12}}^{3}\right)^{\varphi}\right)^{\frac{1}{\varphi}}\right)}\right.\right.$

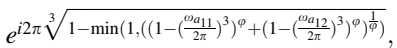
$\left.\left.\sqrt[3]{\min \left(1,\left(k_{a_{11}}^{3 \varphi}+k_{a_{12}}^{3 \varphi}\right)^{\frac{1}{\varphi}}\right)} e^{i 2 \pi} \sqrt[3]{\min \left(1,\left(\left(\frac{\psi_{a 11}}{2 \pi}\right)^{3 \varphi}+\left(\frac{\psi_{a 12}}{2 \pi}\right)^{3 \varphi}\right)^{\frac{1}{\varphi}}\right)}\right)\right\rangle$

\section{Algorithms and applications}

In this section, we clarify the decision-making (DM) process for the constructed model. Firstly, we construct the procedures as shown in Algorithms 1-3 for problems that are described by $\mathrm{CFFNS}_{f} \mathrm{Ss}$. Then, we apply them to real circumstances to get the particular results. 

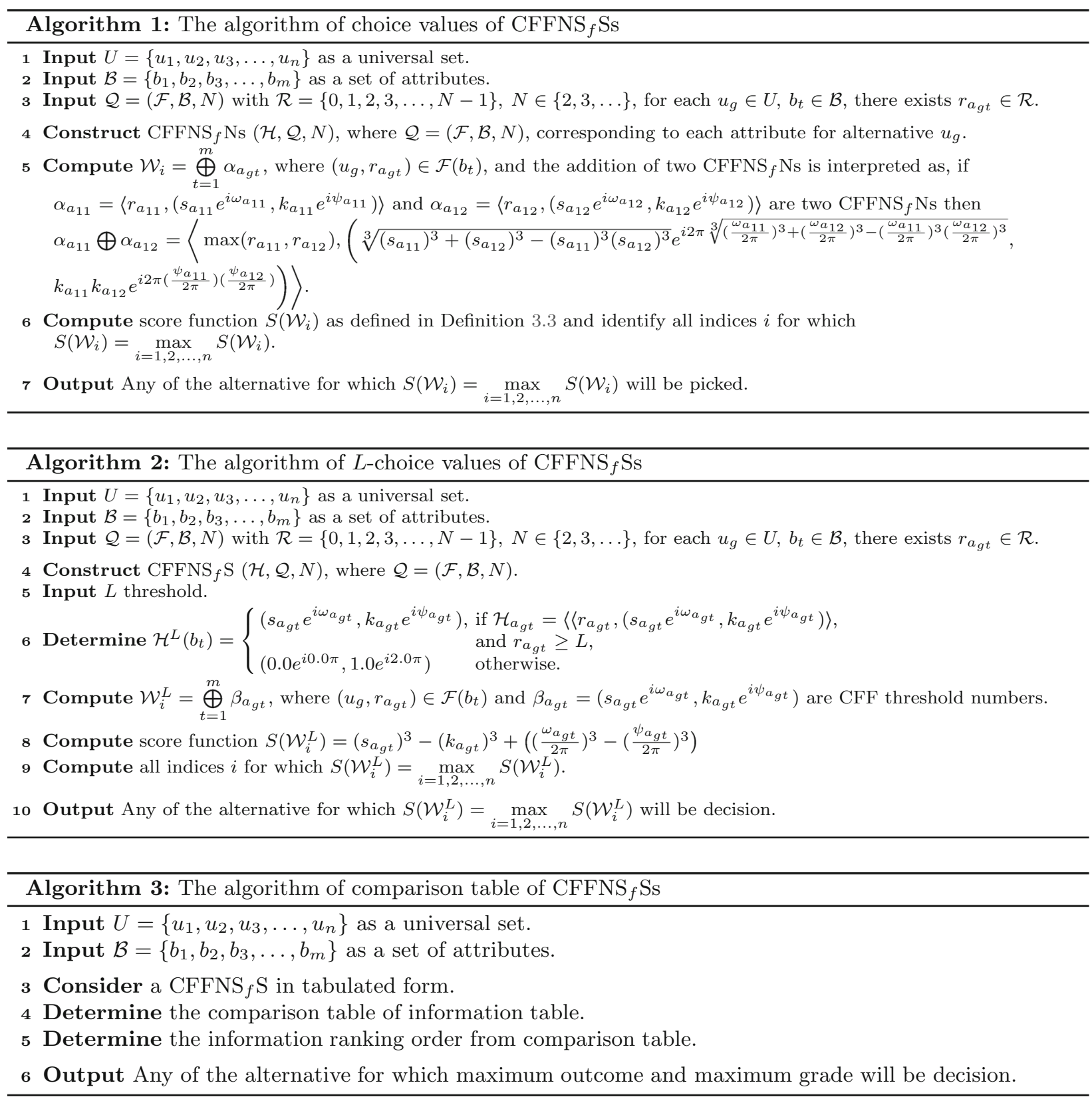

\subsection{Selection of buy new car}

Selection of a car is a difficult task for an auto broker. Productive selection is possible only when there is an essential matching. By choosing the best car, the auto broker will get quality performance. In Example 3.1, different $\mathrm{CFFNS}_{f} \mathrm{Ns}$ for the cars have been defined on the basis of their qualities, by the auto broker. Tabulated form of CFF5S $\mathrm{S}_{f}$ is represented by Table 25.

- Choice value (CV) of CFF5 $\mathrm{S}_{f} \mathrm{~S}$

We can calculate the $\mathrm{CV}$ of $\mathrm{CFF} \mathrm{S}_{f} \mathrm{~S}$ of the car's selection by using Algorithm 1 and calculated results are given in Table 26, where

$$
S\left(\mathcal{W}_{i}\right)=\left(\frac{r_{a_{g t}}}{N-1}\right)^{3}+\left(s_{a_{g t}}\right)^{3}-\left(k_{a_{g t}}\right)^{3}+\left(\left(\frac{\omega_{a_{g t}}}{2 \pi}\right)^{3}-\left(\frac{\psi_{a_{g t}}}{2 \pi}\right)^{3}\right)
$$

From Table 26, it is concluded that according to $S\left(\mathcal{W}_{i}\right)$ values, $x_{3}>x_{4}>x_{2}>x_{1}$ and hence $x_{3}$ has maximum value. So, the auto broker will choose the car $x_{3}$.

\section{- L-Choice value (L-CV) of CFF5 $\mathrm{S}_{f} \mathrm{~S}$}

Now, we will choose the threshold $L$ and will calculate the $\mathrm{CV}$ by using Algorithm 2, where

$$
S\left(\mathcal{W}_{i}^{L}\right)=\left(s_{a_{g t}}\right)^{3}-\left(k_{a_{g t}}\right)^{3}+\left(\left(\frac{\omega_{a_{g t}}}{2 \pi}\right)^{3}-\left(\frac{\psi_{a_{g t}}}{2 \pi}\right)^{3}\right) .
$$

The result is shown by Table 27 . 
Table 25 Tabular form of the $\mathrm{CFF}_{5} \mathrm{~S} S(\mathcal{H}, \mathcal{Q}, 5)$

\begin{tabular}{llll}
\hline$(\mathcal{H}, \mathcal{Q}, 5)$ & $b_{1}$ & $b_{2}$ & $b_{3}$ \\
\hline$x_{1}$ & $\left\langle 2,\left(0.5 e^{i 0.9 \pi}, 0.6 e^{i 1.2 \pi}\right)\right\rangle$ & $\left\langle 3,\left(0.7 e^{i 1.5 \pi}, 0.3 e^{i 0.5 \pi}\right)\right\rangle$ & $\left\langle 1,\left(0.3 e^{i 0.6 \pi}, 0.8 e^{i 1.6 \pi}\right)\right\rangle$ \\
$x_{2}$ & $\left\langle 1,\left(0.3 e^{i 0.5 \pi}, 0.7 e^{i 1.5 \pi}\right)\right\rangle$ & $\left\langle 3,\left(0.8 e^{i 1.6 \pi}, 0.4 e^{i 0.6 \pi}\right)\right\rangle$ & $\left\langle 2,\left(0.6 e^{i 0.9 \pi}, 0.5 e^{i 1.2 \pi}\right)\right\rangle$ \\
$x_{3}$ & $\left\langle 3,\left(0.7 e^{i 1.4 \pi}, 0.3 e^{i 0.5 \pi}\right)\right\rangle$ & $\left\langle 4,\left(0.9 e^{i 1.8 \pi}, 0.1 e^{i 0.3 \pi}\right)\right\rangle$ & $\left\langle 0,\left(0.1 e^{i 0.2 \pi}, 0.9 e^{i 1.9 \pi}\right)\right\rangle$ \\
$x_{4}$ & $\left\langle 0,\left(0.1 e^{i 0.2 \pi}, 0.9 e^{i 1.8 \pi}\right)\right\rangle$ & $\left\langle 2,\left(0.5 e^{i 0.9 \pi}, 0.5 e^{i 1.0 \pi}\right)\right\rangle$ & $\left\langle 4,\left(0.9 e^{i 1.9 \pi}, 0.2 e^{i 0.3 \pi}\right)\right\rangle$ \\
\hline
\end{tabular}

Table 26 Tabular form of $\mathrm{CV}$ of $\mathrm{CFF} \mathrm{S}_{f} \mathrm{~S}(\mathcal{H}, \mathcal{Q}, 5)$

\begin{tabular}{llllll}
\hline$(\mathcal{H}, \mathcal{Q}, 5)$ & $b_{1}$ & $b_{2}$ & $b_{3}$ & $\mathcal{W}_{i}$ & $S\left(\mathcal{W}_{i}\right)$ \\
\hline$x_{1}$ & $\left\langle 2,\left(0.5 e^{i 0.9 \pi}, 0.6 e^{i 1.2 \pi}\right)\right\rangle$ & $\left\langle 3,\left(0.7 e^{i 1.5 \pi}, 0.3 e^{i 0.5 \pi}\right)\right\rangle$ & $\left\langle 1,\left(0.3 e^{i 0.6 \pi}, 0.8 e^{i 1.6 \pi}\right)\right\rangle$ & $\left\langle 3,\left(0.76 e^{i 1.58 \pi}, 0.14 e^{i 0.24 \pi}\right)\right\rangle$ & 1.35 \\
$x_{2}$ & $\left\langle 1,\left(0.3 e^{i 0.5 \pi}, 0.7 e^{i 1.5 \pi}\right)\right\rangle$ & $\left\langle 3,\left(0.8 e^{i 1.6 \pi}, 0.4 e^{i 0.6 \pi}\right)\right\rangle$ & $\left\langle 2,\left(0.6 e^{i 0.9 \pi}, 0.5 e^{i 1.2 \pi}\right)\right\rangle$ & $\left\langle 3,\left(0.86 e^{i 1.65 \pi}, 0.14 e^{i 0.27 \pi}\right)\right\rangle$ & 1.61 \\
$x_{3}$ & $\left\langle 3,\left(0.7 e^{i 1.4 \pi}, 0.3 e^{i 0.5 \pi}\right)\right\rangle$ & $\left\langle 4,\left(0.9 e^{i 1.8 \pi}, 0.1 e^{i 0.3 \pi}\right)\right\rangle$ & $\left\langle 0,\left(0.1 e^{i 0.2 \pi}, 0.9 e^{i 1.9 \pi}\right)\right\rangle$ & $\left\langle 4,\left(0.94 e^{i 1.87 \pi}, 0.03 e^{i 0.07 \pi}\right)\right\rangle$ & 2.65 \\
$x_{4}$ & $\left\langle 0,\left(0.1 e^{i 0.2 \pi}, 0.9 e^{i 1.8 \pi}\right)\right\rangle$ & $\left\langle 2,\left(0.5 e^{i 0.9 \pi}, 0.5 e^{i 1.0 \pi}\right)\right\rangle$ & $\left\langle 4,\left(0.9 e^{i 1.9 \pi}, 0.2 e^{i 0.3 \pi}\right)\right\rangle$ & $\left\langle 4,\left(0.91 e^{i 1.91 \pi}, 0.09 e^{i 0.14 \pi}\right)\right\rangle$ & 2.62 \\
\hline
\end{tabular}

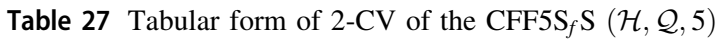

\begin{tabular}{llllll}
\hline$\left(\mathcal{H}^{2}, \mathcal{B}\right)$ & $b_{1}$ & $b_{2}$ & $b_{3}$ & $\mathcal{W}_{i}$ & $S\left(\mathcal{W}_{i}^{L}\right)$ \\
\hline$x_{1}$ & $\left(0.5 e^{i 0.9 \pi}, 0.6 e^{i 1.2 \pi}\right)$ & $\left(0.7 e^{i 1.5 \pi}, 0.3 e^{i 0.5 \pi}\right)$ & $\left(0.0 e^{i 0.0 \pi}, 1.0 e^{i 2.0 \pi}\right)$ & $\left(0.75 e^{i 1.56 \pi}, 0.18 e^{i 0.30 \pi}\right)$ & 0.89 \\
$x_{2}$ & $\left(0.0 e^{i 0.0 \pi}, 1.0 e^{i 2.0 \pi}\right)$ & $\left(0.8 e^{i 1.6 \pi}, 0.4 e^{i 0.6 \pi}\right)$ & $\left(0.6 e^{i 0.9 \pi}, 0.5 e^{i 1.2 \pi}\right)$ & $\left(0.85 e^{i 1.65 \pi}, 0.20 e^{i 0.36 \pi}\right)$ & 1.16 \\
$x_{3}$ & $\left(0.7 e^{i 1.4 \pi}, 0.3 e^{i 0.5 \pi}\right)$ & $\left(0.9 e^{i 1.8 \pi}, 0.1 e^{i 0.3 \pi}\right)$ & $\left(0.0 e^{i 0.0 \pi}, 1.0 e^{i 2.0 \pi}\right)$ & $\left(0.94 e^{i 1.87 \pi}, 0.03 e^{i 0.08 \pi}\right)$ & 1.65 \\
$x_{4}$ & $\left(0.0 e^{i 0.0 \pi}, 1.0 e^{i 2.0 \pi}\right)$ & $\left(0.5 e^{i 0.9 \pi}, 0.5 e^{i 1.0 \pi}\right)$ & $\left(0.9 e^{i 1.9 \pi}, 0.2 e^{i 0.3 \pi}\right)$ & $\left(0.91 e^{i 1.91 \pi}, 0.10 e^{i 0.15 \pi}\right)$ & 1.62 \\
\hline
\end{tabular}

Table 28 Tabular form of the membership and nonmembership values of $\mathrm{CFF}_{5} \mathrm{~S}_{f} \mathrm{~S}$ $(\mathcal{H}, \mathcal{Q}, 5)$

\begin{tabular}{llll}
\hline$(\mathcal{H}, \mathcal{Q}, 5)$ & $b_{1}$ & $b_{2}$ & $b_{3}$ \\
\hline$x_{1}$ & $\left(0.5 e^{i 0.9 \pi}, 0.6 e^{i 1.2 \pi}\right)$ & $\left(0.7 e^{i 1.5 \pi}, 0.3 e^{i 0.5 \pi}\right)$ & $\left(0.3 e^{i 0.6 \pi}, 0.8 e^{i 1.6 \pi}\right)$ \\
$x_{2}$ & $\left(0.3 e^{i 0.5 \pi}, 0.7 e^{i 1.5 \pi}\right)$ & $\left(0.8 e^{i 1.6 \pi}, 0.4 e^{i 0.6 \pi}\right)$ & $\left(0.6 e^{i 0.9 \pi}, 0.5 e^{i 1.2 \pi}\right)$ \\
$x_{3}$ & $\left(0.7 e^{i 1.4 \pi}, 0.3 e^{i 0.5 \pi}\right)$ & $\left(0.9 e^{i 1.8 \pi}, 0.1 e^{i 0.3 \pi}\right)$ & $\left(0.1 e^{i 0.2 \pi}, 0.9 e^{i 1.9 \pi}\right)$ \\
$x_{4}$ & $\left(0.1 e^{i 0.2 \pi}, 0.9 e^{i 1.8 \pi}\right)$ & $\left(0.5 e^{i 0.9 \pi}, 0.5 e^{i 1.0 \pi}\right)$ & $\left(0.9 e^{i 1.9 \pi}, 0.2 e^{i 0.3 \pi}\right)$ \\
\hline
\end{tabular}

In Table 27, we took $L=2$ for DM and get the 2-CV of $\mathrm{CFF}_{5} \mathrm{~S}_{f} \mathrm{~S}$. It can observe from Table 27 that the car $x_{3}$ has highest output value. So, $x_{3}$ will be selected by the auto broker.

\section{- Comparison table of CFF5 $\mathrm{S}_{f} \mathrm{~S}$}

Comparison table is a square table in which rows and columns are represented by the name of objects of universe such as $u_{1}, u_{2}, u_{3}, \ldots, u_{n}$ and $q_{g t}=$ the CFFNS $_{f}$ values of the attributes for which the value of score function of $u_{d} \geq u_{j}$.

Membership and non-membership values of Table 25 are given in tabular form in Table 28.

The comparison table of Table 28 is given by Table 29 .

The result will be derived by subtracting the row and column sum of Table 29.

From Table 30, it is concluded that the highest rank and grade sum is 3 and 7 , respectively, which is obtained by $x_{3}$. So, $x_{3}$ car is selected by the auto broker.

\subsection{Selection of the best cellular telecommunication company in Pakistan}

Since due to COVID-19, everything has shifted to the online mode. So, the usage of the internet has increased. Due to that, in market, the competition among different network provider companies has been tough day by day. All companies are presenting different internet packages according to the needs

Table 29 Comparison table of $\mathrm{CFF}_{f} \mathrm{~S}(\mathcal{H}, \mathcal{Q}, 5)$

\begin{tabular}{lllll}
\hline & $x_{1}$ & $x_{2}$ & $x_{3}$ & $x_{4}$ \\
\hline$x_{1}$ & 3 & 1 & 1 & 2 \\
$x_{2}$ & 2 & 3 & 1 & 2 \\
$x_{3}$ & 2 & 2 & 3 & 2 \\
$x_{4}$ & 1 & 1 & 1 & 3 \\
\hline
\end{tabular}


Table 30 Ranking Table

\begin{tabular}{lllll}
\hline & Grade sum $\left(G_{s}\right)$ & Row sum $\left(R_{s}\right)$ & Column sum $\left(C_{s}\right)$ & Final Rank $\left(R_{s}-C_{s}\right)$ \\
\hline$x_{1}$ & 6 & 7 & 8 & -1 \\
$x_{2}$ & 6 & 8 & 7 & 1 \\
$x_{3}$ & 7 & 9 & 6 & 3 \\
$x_{4}$ & 6 & 6 & 9 & -3 \\
\hline
\end{tabular}

of the customers. So, it's a difficult task for customers to choose the sim card of the best telecommunication company. Suppose that a student, in Pakistan, decides to purchase a new sim card to attend online classes. Before buying the sim, the student has collected some relevant rating based information related to the internet packages and internet speed about five different network companies such as Zong, Ufone, Telenor, Jazz, and Warid. Each network company has different prices of internet packages depending on time as well. Let $Y=\left\{y_{1}=\right.$ zong, $y_{2}=\mathrm{SCOM}, \quad y_{3}=$ telenor, $y_{4}=$ jazz, $y_{5}=$ ufone $\}$ be a universal set and $\mathcal{O}=\left\{o_{1}=\right.$ $3 \mathrm{G} / 4$ Gspeed, $\quad o_{2}=$ Packagestimeduration, $\quad o_{3}=$ Monthlycost, $o_{4}=$ Signalstrength $\} \subseteq \mathcal{A}$ be a set of attributes, which are used to assign grades to network companies. The ratings are on the basis of internet packages and speed provided in last year and users reviews. It may be noted that the ranking of alternatives with respect to parameters may get affected and altered if the time and location is different for a particular network company. The initial survey is organized in Table 31, where:

- five represents 'outstanding',

- four represents 'very good',

- three $\checkmark$ represents 'good',

- two $\diamond$ represents 'average',

- one represents 'subpar',

- $\diamond$ represents 'poor'.

The set of grades $\mathcal{R}=\{0,1,2,3,4,5\}$ can be easily associated with $\diamond$ and $\diamond$ as follows:

Table 31 Information extracted from the related data

\begin{tabular}{|c|c|c|c|c|}
\hline$Y / \mathcal{O}$ & $o_{1}$ & $o_{2}$ & $o_{3}$ & $o_{4}$ \\
\hline$y_{1}$ & 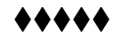 & 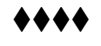 & $\diamond \diamond$ & 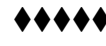 \\
\hline$y_{2}$ & $\diamond$ & $\diamond$ & $\diamond$ & $\diamond$ \\
\hline$y_{3}$ & $\leftrightarrow \bullet$ & 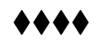 & $\diamond \diamond$ & $\diamond$ \\
\hline$y_{4}$ & 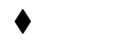 & $\diamond$ & $\diamond \diamond$ & $\diamond$ \\
\hline$y_{5}$ & $\diamond$ & $\diamond$ & $\diamond$ & 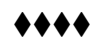 \\
\hline
\end{tabular}

- 0 stands for ' $\diamond$ ',

- 1 stands for ' $\nabla$ ',

- 2 stands for ' $\boldsymbol{\wedge}$ ',

- 3 stands for ' $\diamond$

- 4 stands for ' $\Delta\rangle\langle\rangle$ ',

- 5 stands for '

Based on the overall qualities of the network companies, the student gives evaluation scores to the sim cards which is shown as Table 31 and the tabular representation of its associated 6-soft set is given in Table 32 .

Although it is easy to extract the grade data in actual information, the data possess the fuzzy uncertainty characteristics. In order to address the ambiguity of data, we construct $\mathrm{CFFNS}_{f} \mathrm{~S}$ by using a certain grade. This evaluation of sim cards by students complies with the guidelines as follows:

$$
\begin{aligned}
-2.0 & \leq S(Y)<-1.4 \text { when grade } 0, \\
-1.4 & \leq S(Y)<-0.5 \text { when grade } 1, \\
-0.5 & \leq S(Y)<0.0 \text { when grade } 2, \\
0.0 & \leq S(Y)<0.5 \text { when grade } 3, \\
0.5 & \leq S(Y)<1.4 \text { when grade } 4, \\
1.4 & \leq S(Y) \leq 2.0 \text { when grade } 5 .
\end{aligned}
$$

By Definition 3.1, the $\operatorname{CFF}_{6} \mathrm{~S} S(\mathcal{H}, \mathcal{Q}, 6)$ can be defined as follows:

The $\mathrm{CFF}_{6} \mathrm{~S}_{f} \mathrm{~S}(\mathcal{H}, \mathcal{Q}, 6)$ can be represented more clearly in tabular form shown as in Table 33.

\section{- Choice value (CV) of CFF6 $\mathrm{S}_{f} \mathrm{~S}$}

We can calculate the $\mathrm{CV}$ of $\mathrm{CFF}_{6} \mathrm{~S}_{f} \mathrm{~S}$ of the sim card's selection by using Algorithm 1 as given by Table 34, where

Table 32 Tabular form of the 6soft set $(\mathcal{F}, \mathcal{B}, 6)$

\begin{tabular}{lllll}
\hline$(\mathcal{F}, \mathcal{B}, 6)$ & $o_{1}$ & $o_{2}$ & $o_{3}$ & $o_{4}$ \\
\hline$y_{1}$ & 5 & 4 & 3 & 5 \\
$y_{2}$ & 1 & 2 & 2 & 1 \\
$y_{3}$ & 3 & 4 & 3 & 2 \\
$y_{4}$ & 0 & 2 & 3 & 2 \\
$y_{5}$ & 2 & 1 & 0 & 4 \\
\hline
\end{tabular}




$$
\begin{aligned}
h\left(o_{1}\right)= & \left\{\left\langle\left(y_{1}, 5\right), 0.95 e^{i 1.78 \pi}, 0.05 e^{i 0.24 \pi}\right\rangle,\left\langle\left(y_{2}, 1\right), 0.32 e^{i 0.54 \pi}, 0.89 e^{i 1.74 \pi}\right\rangle,\left\langle\left(y_{3}, 3\right), 0.48 e^{i 1.06 \pi}, 0.17 e^{i 0.30 \pi}\right\rangle,\right. \\
& \left.\left.\left\langle\left(y_{4}, 0\right), 0.21 e^{i 0.04 \pi}, 0.83 e^{i 1.94 \pi}\right\rangle\right\},\left\langle\left(y_{5}, 2\right), 0.29 e^{i 0.84 \pi}, 0.57 e^{i 1.16 p i}\right\rangle\right\}, \\
h\left(o_{2}\right)= & \left\{\left\langle\left(y_{1}, 4\right), 0.76 e^{i 1.69 \pi}, 0.39 e^{i 0.29 \pi}\right\rangle,\left\langle\left(y_{2}, 2\right), 0.24 e^{i 0.64 \pi}, 0.43 e^{i 1.22 \pi}\right\rangle,\left\langle\left(y_{3}, 4\right), 0.7 e^{i 1.71 \pi}, 0.32 e^{i 0.21 \pi}\right\rangle,\right. \\
& \left.\left.\left\langle\left(y_{4}, 2\right), 0.26 e^{i 0.56 \pi}, 0.43 e^{i 1.21 \pi}\right\rangle\right\},\left\langle\left(y_{5}, 1\right), 0.34 e^{i 0.84 \pi}, 0.95 e^{i 1.71 \pi}\right\rangle\right\}, \\
h\left(o_{3}\right)= & \left\{\left\langle\left(y_{1}, 3\right), 0.58 e^{i 1.38 \pi}, 0.23 e^{i 0.52 \pi}\right\rangle,\left\langle\left(y_{2}, 2\right), 0.29 e^{i 0.54 \pi}, 0.49 e^{i 0.98 \pi}\right\rangle,\left\langle\left(y_{3}, 3\right), 0.52 e^{i 1.44 \pi}, 0.32 e^{i 0.72 \pi}\right\rangle,\right. \\
& \left.\left.\left\langle\left(y_{4}, 3\right), 0.69 e^{i 1.28 \pi}, 0.45 e^{i 0.86 \pi}\right\rangle\right\},\left\langle\left(y_{5}, 0\right), 0.07 e^{i 0.24 \pi}, 0.99 e^{i 1.88 \pi}\right\rangle\right\}, \\
h\left(o_{4}\right)= & \left\{\left\langle\left(y_{1}, 5\right), 0.91 e^{i 1.93 \pi}, 0.02 e^{i 0.05 \pi}\right\rangle,\left\langle\left(y_{2}, 1\right), 0.43 e^{i 0.66 \pi}, 0.85 e^{i 1.46 \pi}\right\rangle,\left\langle\left(y_{3}, 2\right), 0.44 e^{i 0.44 \pi}, 0.35 e^{i 1.08 \pi}\right\rangle,\right. \\
& \left.\left.\left\langle\left(y_{4}, 2\right), 0.19 e^{i 0.38 \pi}, 0.37 e^{i 1.06 \pi}\right\rangle\right\},\left\langle\left(y_{5}, 4\right), 0.81 e^{i 1.53 \pi}, 0.12 e^{i 0.34 \pi}\right\rangle\right\} .
\end{aligned}
$$

$S\left(\mathcal{W}_{i}\right)=\left(\frac{r_{a_{g t}}}{N-1}\right)^{3}+\left(s_{a_{g t}}\right)^{3}-\left(k_{a_{g t}}\right)^{3}+\left(\left(\frac{\omega_{a_{g t}}}{2 \pi}\right)^{3}-\left(\frac{\psi_{a_{g t}}}{2 \pi}\right)^{3}\right)$.

From Table 34, it is concluded that according to $S\left(\mathcal{W}_{i}\right)$, $y_{1}>y_{3}>y_{5}>y_{4}>y_{2}$ and hence $y_{1}$ has maximum value. So, the student will choose the sim card of zong.

\section{- L-Choice value (L-CV) of CFF6 $\mathrm{S}_{f} \mathrm{~S}$}

Now, by using the second procedure as given by Algorithm 2, $L$ value will be chosen and the results are given by Table 35, where

$S\left(\mathcal{W}_{i}^{L}\right)=\left(s_{a_{g t}}\right)^{3}-\left(k_{a_{g t}}\right)^{3}+\left(\left(\frac{\omega_{a_{g t}}}{2 \pi}\right)^{3}-\left(\frac{\psi_{a_{g t}}}{2 \pi}\right)^{3}\right)$.

In Table 35, we have chosen $L=3$ for DM and get the 3-CV of $\mathrm{CFF}_{6} \mathrm{~S}_{f} \mathrm{~S}$. It can observe from Table 35 that the telecommunication company $y_{1}=$ zong has highest output value. So, $y_{1}$ will be selected by the student.

\section{- Comparison table of CFF6 $\mathrm{S}_{f} \mathrm{~S}$}

Now to apply the third procedure as shown in Algorithm 3, membership and non-membership values of Table 33 are given by Table 36 .

The comparison table of Table 36 is given by Table 37 .

The result will be derived by subtracting the row and column sum of Table 37.
From Table 38, it is concluded that the highest rank and grade sum is 17 and 16, respectively, which is obtained by $y_{1}=$ zong. So, sim card $y_{1}$ is selected by the student.

\section{Comparison analysis}

To certify the rationality of our proposed model, we solve the same example "Selection of the best cellular telecommunication company in Pakistan" using $\mathrm{FFY}_{w} \mathrm{~A}$ (Garg et al. 2020) and $\mathrm{FFY}_{w} \mathrm{G}$ (Garg et al. 2020) operators:

- Step 1. The membership and non-membership terms of amplitude part are the same as given in Table 33, but their grades have neglected and phase terms in all $\mathrm{CFFNS}_{f}$ Ns have taken to be zero given by Table 39 .

- Step 2. Let $\tau_{k}=(0.2,0.4,0.1,0.3)^{t}$ is the weight vector on alternatives.

- Step 3. The entries of aggregated values $W_{i}$ of the alternatives by using $\mathrm{FFY}_{w} \mathrm{~A}$ (Garg et al. 2020) operator defined as follows:

Table 33 Tabular form of the $\operatorname{CFF}_{6} \mathrm{~S}(\mathcal{H}, \mathcal{Q}, 6)$

\begin{tabular}{lllll}
\hline$(\mathcal{H}, \mathcal{Q}, 6)$ & $o_{1}$ & $o_{2}$ & $o_{3}$ & $o_{4}$ \\
\hline$y_{1}$ & $\left\langle 5,\left(0.95 e^{i 1.78 \pi}, 0.05 e^{i 0.24 \pi}\right)\right\rangle$ & $\left\langle 4,\left(0.76 e^{i 1.69 \pi}, 0.39 e^{i 0.29 \pi}\right)\right\rangle$ & $\left\langle 3,\left(0.58 e^{i 1.38 \pi}, 0.23 e^{i 0.52 \pi}\right)\right\rangle$ & $\left\langle 5,\left(0.91 e^{i 1.93 \pi}, 0.02 e^{i 0.05 \pi}\right)\right\rangle$ \\
$y_{2}$ & $\left\langle 1,\left(0.32 e^{i 0.54 \pi}, 0.89 e^{i 1.74 \pi}\right)\right\rangle$ & $\left\langle 2,\left(0.24 e^{i 0.64 \pi}, 0.43 e^{i 1.22 \pi}\right)\right\rangle$ & $\left\langle 2,\left(0.29 e^{i 0.54 \pi}, 0.49 e^{i 0.98 \pi}\right)\right\rangle$ & $\left\langle 1,\left(0.43 e^{i 0.66 \pi}, 0.85 e^{i 1.46 \pi}\right)\right\rangle$ \\
$y_{3}$ & $\left\langle 3,\left(0.48 e^{i 1.06 \pi}, 0.17 e^{i 0.30 \pi}\right)\right\rangle$ & $\left\langle 4,\left(0.70 e^{i 1.71 \pi}, 0.32 e^{i 0.21 \pi}\right)\right\rangle$ & $\left\langle 3,\left(0.52 e^{i 1.44 \pi}, 0.32 e^{i 0.72 \pi}\right)\right\rangle$ & $\left\langle 2,\left(0.44 e^{i 0.44 \pi}, 0.35 e^{i 1.08 \pi}\right)\right\rangle$ \\
$y_{4}$ & $\left\langle 0,\left(0.21 e^{i 0.04 \pi}, 0.83 e^{i 1.94 \pi}\right)\right\rangle$ & $\left\langle 2,\left(0.26 e^{i 0.56 \pi}, 0.43 e^{i 1.21 \pi}\right)\right\rangle$ & $\left\langle 3,\left(0.69 e^{i 1.28 \pi}, 0.45 e^{i 0.86 \pi}\right)\right\rangle$ & $\left\langle 2,\left(0.19 e^{i 0.38 \pi}, 0.37 e^{i 1.06 \pi}\right)\right\rangle$ \\
$y_{5}$ & $\left\langle 2,\left(0.29 e^{i 0.84 \pi}, 0.57 e^{i 1.16 \pi}\right)\right\rangle$ & $\left\langle 1,\left(0.34 e^{i 0.84 \pi}, 0.95 e^{i 1.71 \pi}\right)\right\rangle$ & $\left\langle 0,\left(0.07 e^{i 0.24 \pi}, 0.99 e^{i 1.88 \pi}\right)\right\rangle$ & $\left\langle 4,\left(0.81 e^{i 1.53 \pi}, 0.12 e^{i 0.34 \pi}\right)\right\rangle$ \\
\hline
\end{tabular}



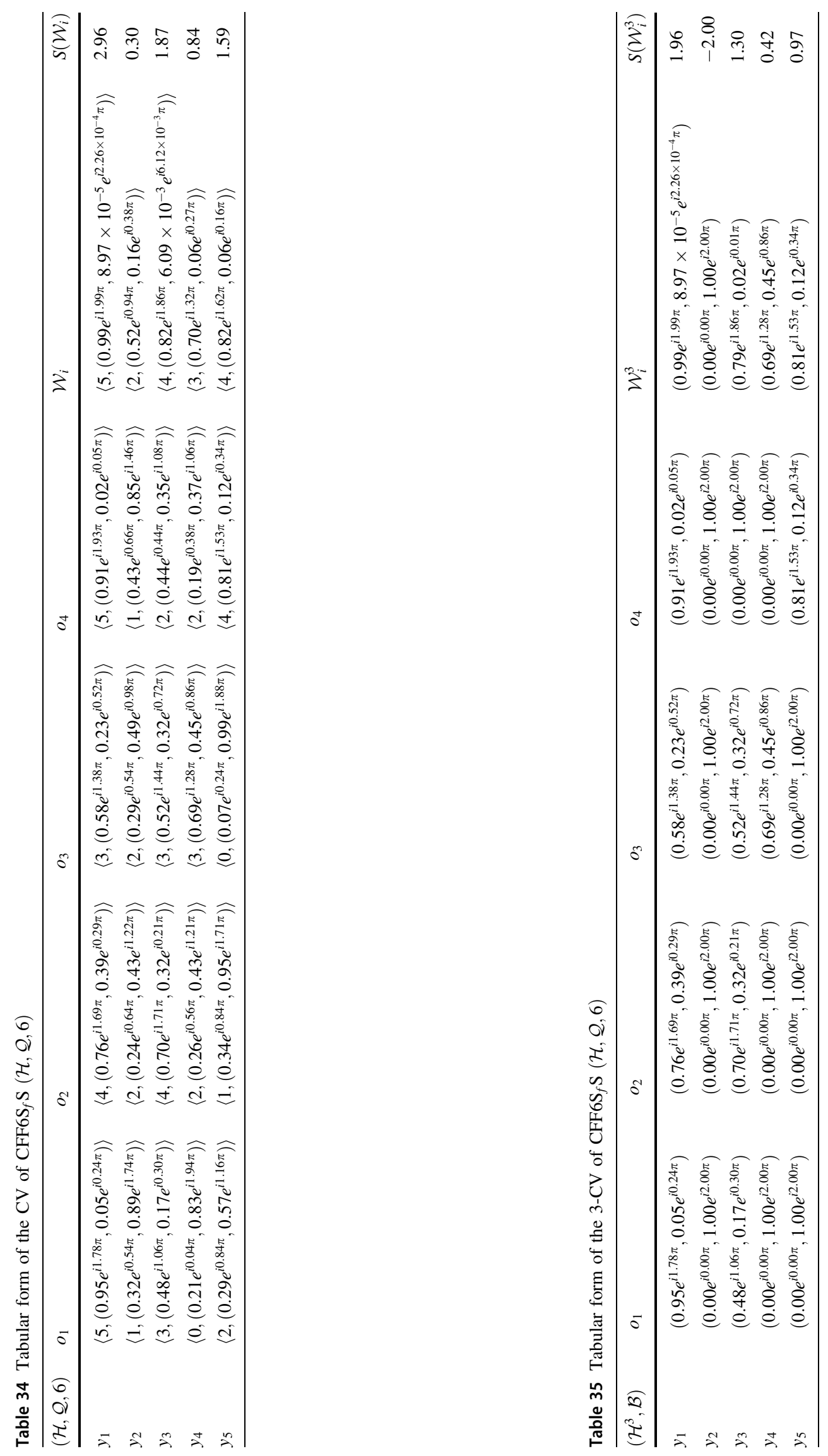


\subsection{Discussion}

1. We present a comparative study with existing MADM techniques, namely, $\mathrm{FFY}_{w} \mathrm{~A}$ and $\mathrm{FFY}_{w} \mathrm{G}$ operators

$\mathcal{W}_{i}=\left\langle\sqrt[3]{\min \left(1,\left(\sum_{t=1}^{n}\left(\tau_{t} s_{g t}^{3 \varsigma}\right)\right)^{\frac{1}{\varsigma}}\right)}, \sqrt[3]{1-\min \left(1,\left(\sum_{t=1}^{n}\left(\tau_{t}\left(1-k_{g t}^{3}\right)^{\varsigma}\right)\right)^{\frac{1}{\varsigma}}\right)}\right\rangle$

For $\varsigma=3$, the values are:

$\mathcal{W}_{1}=(0.87,0.29)$

$\mathcal{W}_{2}=(0.38,0.64)$

$\mathcal{W}_{3}=(0.64,0.31)$

$\mathcal{W}_{4}=(0.53,0.51)$

$\mathcal{W}_{5}=(0.71,0.64)$

- Step 4. The entries of aggregated values $\mathcal{W}_{i}$ of the alternatives by using FFY $_{w}$ G (Garg et al. 2020) operator defined as follows: which manifest the proficiency and adeptness of proposed methods. The ranking of alternatives by applying the proposed and compared techniques are given in Table 41.

2. According to Table 41 , Zong $\left(y_{1}\right)$ is the best alternative obtained from the extant and proposed model which shows the validity and authenticity of proposed MADM methods.

3. Figure 1 skillfully depicts the comparison between the outcomes of proposed and existing decision-making methodologies by displaying an illustrated bar

$\mathcal{W}_{i}=\left\langle\sqrt[3]{1-\min \left(1,\left(\sum_{t=1}^{n}\left(\tau_{t}\left(1-s_{g t}^{3}\right)^{\phi}\right)\right)^{\frac{1}{\phi}}\right)}, \sqrt[3]{\min \left(1,\left(\sum_{t=1}^{n}\left(\tau_{t} k_{g t}^{3 \phi}\right)^{\frac{1}{\phi}}\right)\right)}\right\rangle$

For $\phi=3$, the values are:

$$
\begin{aligned}
& \mathcal{W}_{1}=(0.79,0.35) \\
& \mathcal{W}_{2}=(0.33,0.80) \\
& \mathcal{W}_{3}=(0.57,0.33) \\
& \mathcal{W}_{4}=(0.33,0.69) \\
& \mathcal{W}_{5}=(0.50,0.89)
\end{aligned}
$$

- Step 5. The score of each executed value from $\mathrm{FFY}_{w} \mathrm{~A}$ and $\mathrm{FFY}_{w} \mathrm{G}$ operators are assembled in Table 40. chart among network companies and their order of ranking, demonstrating the consistency and competency of the presented MADM techniques.

Table 37 Comparison table of $\mathrm{CFF}_{f} \mathrm{~S}(\mathcal{H}, \mathcal{Q}, 6)$

\begin{tabular}{llllll}
\hline & $y_{1}$ & $y_{2}$ & $y_{3}$ & $y_{4}$ & $y_{5}$ \\
\hline$y_{1}$ & 4 & 4 & 4 & 4 & 4 \\
$y_{2}$ & 0 & 4 & 0 & 2 & 2 \\
$y_{3}$ & 0 & 4 & 4 & 4 & 3 \\
$y_{4}$ & 0 & 2 & 0 & 4 & 2 \\
$y_{5}$ & 0 & 2 & 1 & 2 & 4 \\
\hline
\end{tabular}

Table 36 Tabular form of the membership and non-membership values of $\mathrm{CFF}_{6} \mathrm{~S} S(\mathcal{H}, \mathcal{Q}, 6)$

\begin{tabular}{lllll}
\hline$(\mathcal{H}, \mathcal{Q}, 6)$ & $o_{1}$ & $o_{2}$ & $o_{3}$ & $o_{4}$ \\
\hline$y_{1}$ & $\left(0.95 e^{i 1.78 \pi}, 0.05 e^{i 0.24 \pi}\right)$ & $\left(0.76 e^{i 1.69 \pi}, 0.39 e^{i 0.29 \pi}\right)$ & $\left(0.58 e^{i 1.38 \pi}, 0.23 e^{i 0.52 \pi}\right)$ & $\left(0.91 e^{i 1.93 \pi}, 0.02 e^{i 0.05 \pi}\right)$ \\
$y_{2}$ & $\left(0.32 e^{i 0.54 \pi}, 0.89 e^{i 1.74 \pi}\right)$ & $\left(0.24 e^{i 0.64 \pi}, 0.43 e^{i 1.22 \pi}\right)$ & $\left(0.29 e^{i 0.54 \pi}, 0.49 e^{i 0.98 \pi}\right)$ & $\left(0.43 e^{i 0.66 \pi}, 0.85 e^{i 1.46 \pi}\right)$ \\
$y_{3}$ & $\left(0.48 e^{i 1.06 \pi}, 0.17 e^{i 0.30 \pi}\right)$ & $\left(0.70 e^{i 1.71 \pi}, 0.32 e^{i 0.21 \pi}\right)$ & $\left(0.52 e^{i 1.44 \pi}, 0.32 e^{i 0.72 \pi}\right)$ & $\left(0.44 e^{i 0.44 \pi}, 0.35 e^{i 1.08 \pi}\right)$ \\
$y_{4}$ & $\left(0.21 e^{i 0.04 \pi}, 0.83 e^{i 1.94 \pi}\right)$ & $\left(0.26 e^{i 0.56 \pi}, 0.43 e^{i 1.21 \pi}\right)$ & $\left(0.69 e^{i 1.28 \pi}, 0.45 e^{i 0.86 \pi}\right)$ & $\left(0.19 e^{i 0.38 \pi}, 0.37 e^{i 1.06 \pi}\right)$ \\
$y_{5}$ & $\left(0.29 e^{i 0.84 \pi}, 0.57 e^{i 1.16 \pi}\right)$ & $\left(0.34 e^{i 0.84 \pi}, 0.95 e^{i 1.71 \pi}\right)$ & $\left(0.07 e^{i 0.24 \pi}, 0.99 e^{i 1.88 \pi}\right)$ & $\left(0.81 e^{i 1.53 \pi}, 0.12 e^{i 0.34 \pi}\right)$ \\
\hline
\end{tabular}


Table 38 Ranking Table

\begin{tabular}{llllc}
\hline & Grade sum $\left(G_{s}\right)$ & Row sum $\left(R_{s}\right)$ & Column sum $\left(C_{s}\right)$ & Final rank $\left(R_{s}-C_{s}\right)$ \\
\hline$y_{1}$ & 17 & 20 & 4 & 16 \\
$y_{2}$ & 6 & 8 & 16 & -8 \\
$y_{3}$ & 12 & 15 & 9 & 6 \\
$y_{4}$ & 7 & 8 & 16 & -8 \\
$y_{5}$ & 7 & 9 & 15 & -6 \\
\hline
\end{tabular}

Table 39 Fermatean fuzzy decision matrix

\begin{tabular}{lllll}
\hline$Y / \mathcal{O}$ & $o_{1}$ & $o_{2}$ & $o_{4}$ \\
\hline$y_{1}$ & $(0.95,0.05)$ & $(0.76,0.39)$ & $(0.58,0.23)$ & $(0.91,0.02)$ \\
$y_{2}$ & $(0.32,0.89)$ & $(0.24,0.43)$ & $(0.29,0.49)$ & $(0.43,0.85)$ \\
$y_{3}$ & $(0.48,0.17)$ & $(0.70,0.32)$ & $(0.52,0.32)$ & $(0.44,0.35)$ \\
$y_{4}$ & $(0.21,0.83)$ & $(0.26,0.43)$ & $(0.69,0.45)$ & $(0.19,0.37)$ \\
$y_{5}$ & $(0.29,0.57)$ & $(0.34,0.95)$ & $(0.07,0.99)$ & $(0.81,0.12)$ \\
\hline
\end{tabular}

4. Our proposed approaches provide the most comprehensive and adaptable methodologies because they effectively incorporate the existing proficient MADM methods, namely, $\mathrm{FFY}_{w} \mathrm{~A}$ operators and $\mathrm{FFY}_{w} \mathrm{G}$ operators, by taking phase term equal to zero and neglecting the parameterized grading of alternatives. On the contrary, existing techniques cannot handle the twodimensional parameterized fuzzy information. They are designed to deal with one-dimensional information only.

\section{Development of CFFNS $_{f}$-TOPSIS method for MAGDM problems}

In this section, we aim to renovate the TOPSIS approach for the environment of $\mathrm{CFFNS}_{f}$ to account for MAGDM problems. The chief idea of the proposed CFFNS $_{f}$-TOPSIS technique is to find the most appropriate alternative having maximum distance from negative ideal solution (NIS) and shortest distance from positive ideal solution (PIS). Mathematical steps of MAGDM are as follows:

Let $\mathfrak{D}=\left\{\mathfrak{D}_{1}, \mathfrak{D}_{2}, \ldots, \mathfrak{D}_{l}\right\}$ be the set of $l$ distinct experts which are appointed for the selection of best alternative from $\mathfrak{I}=\left\{\mathfrak{I}_{1}, \mathfrak{I}_{2}, \mathfrak{I}_{3}, \ldots, \mathfrak{I}_{n}\right\}$ relating to some specific attributes. $\mathfrak{B}=\left\{\mathfrak{B}_{1}, \mathfrak{B}_{2}, \mathfrak{B}_{3}, \ldots, \mathfrak{B}_{m}\right\}$ represents the collection of attributes which is selected by the experts according to the necessities of decision-making problem and $\zeta=\left(\zeta_{1}, \zeta_{2}, \ldots, \zeta_{l}\right)^{T}$ be the weight vector, represents the weightage of experts such that $\sum_{c=1}^{l} \zeta_{c}=1$.

The step-by-step procedure of $\mathrm{CFFNS}_{f}$-TOPSIS method is as follows:

- Step 1. Firstly, decision-makers will give grades corresponding to the linguistic terms according to the importance of alternatives on the basis of attributes. Then each expert $\mathfrak{D}_{\mathfrak{c}}$ will assign $\mathrm{CFFNS}_{f} \mathrm{~N}$,

Table 40 Score values

\begin{tabular}{llllrrl}
\hline Methods & $S\left(\mathcal{W}_{1}\right)$ & $S\left(\mathcal{W}_{2}\right)$ & $S\left(\mathcal{W}_{3}\right)$ & $S\left(\mathcal{W}_{4}\right)$ & $S\left(\mathcal{W}_{5}\right)$ & Best alternative \\
\hline FFY $_{w}$ A operator & 0.63 & -0.21 & 0.23 & 0.02 & 0.10 & $y_{1}$ \\
FFY $_{w}$ G operator & 0.45 & -0.48 & 0.15 & -0.29 & -0.58 & $y_{1}$ \\
\hline
\end{tabular}

Table 41 Comparison analysis with existing methods

\begin{tabular}{lccccccc}
\hline Methods & $S\left(\mathcal{W}_{1}\right)$ & $S\left(\mathcal{W}_{2}\right)$ & $S\left(\mathcal{W}_{3}\right)$ & $S\left(\mathcal{W}_{4}\right)$ & $S\left(\mathcal{W}_{5}\right)$ & Ranking order & Best alternative \\
\hline CV of $\mathrm{CFF}_{f} \mathrm{~S}$ (proposed) & 2.96 & 0.30 & 1.87 & 0.84 & 1.59 & $y_{1}>y_{3}>y_{5}>y_{4}>y_{2}$ & $y_{1}$ \\
3-CV of $\mathrm{CFF}_{f} \mathrm{~S}$ (proposed) & 1.96 & -2.00 & 1.30 & 0.42 & 0.97 & $y_{1}>y_{3}>y_{5}>y_{4}>y_{2}$ & $y_{1}$ \\
FFY ${ }_{w}$ A operator (Garg et al. 2020) & 0.63 & -0.21 & 0.23 & 0.02 & 0.10 & $y_{1}>y_{3}>y_{5}>y_{4}>y_{2}$ & $y_{1}$ \\
FFY ${ }_{w}$ G operator (Garg et al. 2020) & 0.45 & -0.48 & 0.15 & -0.29 & -0.58 & $y_{1}>y_{3}>y_{4}>y_{2}>y_{5}$ & $y_{1}$ \\
\hline
\end{tabular}


Fig. 1 Comparison chart

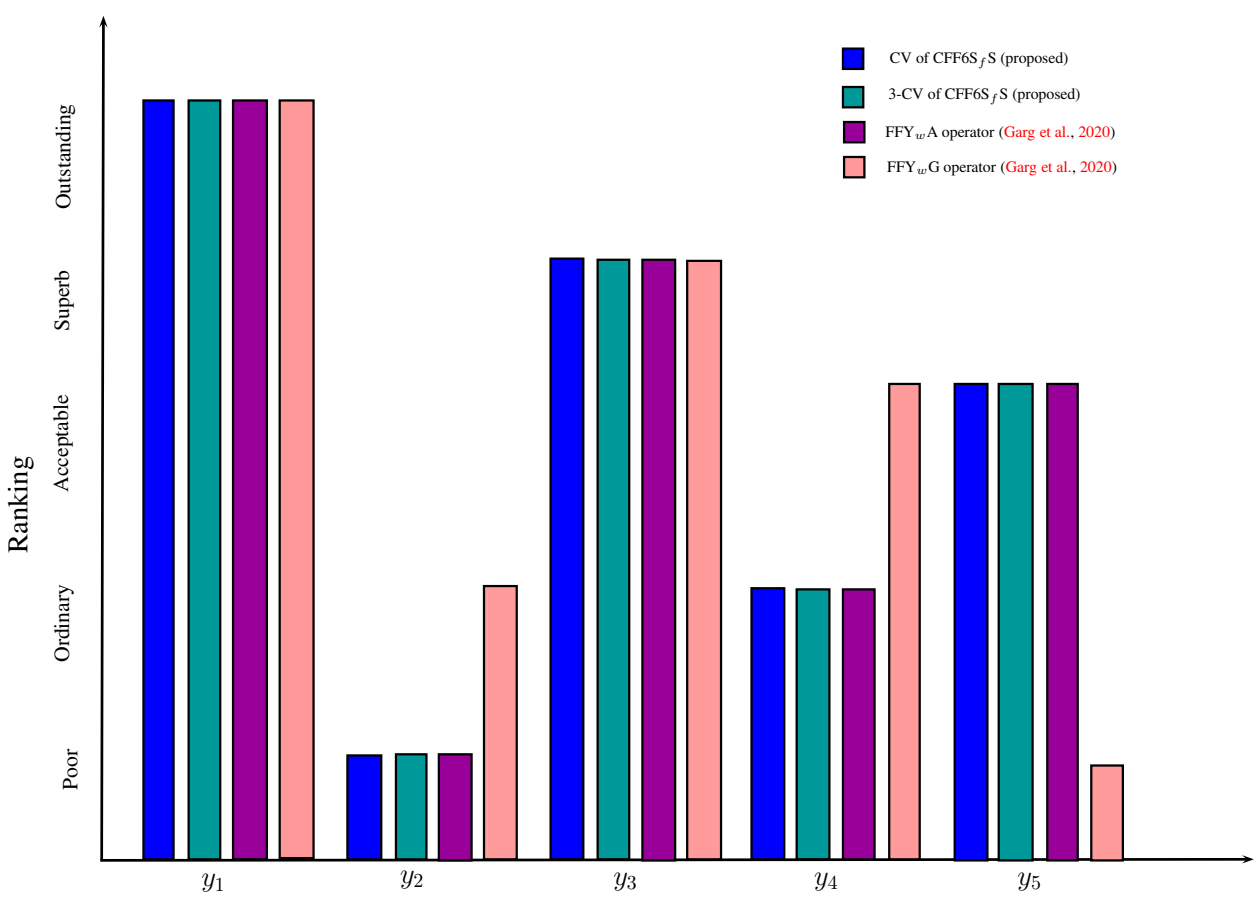

Telecommunication Companies in Pakistan

corresponding to each grade in $\operatorname{NS}_{f} \mathrm{~S}\left(\mathcal{H}^{(\mathfrak{c})}, \mathcal{Q}, N\right)$, according to the defined criteria for ranking. The $\mathrm{CFFNS}_{f} \mathrm{Ns}$ allocated by the decision-maker $\mathfrak{D}_{\mathfrak{c}}$ are adapted in complex Fermatean fuzzy $\mathrm{NS}_{f}$ decision matrix $\left(\mathrm{CFFNS}_{f} \mathrm{DM}\right) \quad \boldsymbol{( \mathfrak { G }}^{(\mathfrak{c})}=\left(\boldsymbol{\mathfrak { G }}_{g t}^{(\mathfrak{c})}\right)_{n \times m}$. Hence, $\mathrm{CFFNS}_{f}$ DMs $\boldsymbol{\mathfrak { 5 }}^{(1)}, \mathfrak{5}^{(2)}, \ldots, \boldsymbol{6}^{(l)}$ are arranged by $l$ decision-experts as follows:

$\mathfrak{5}^{(\mathfrak{c})}=$

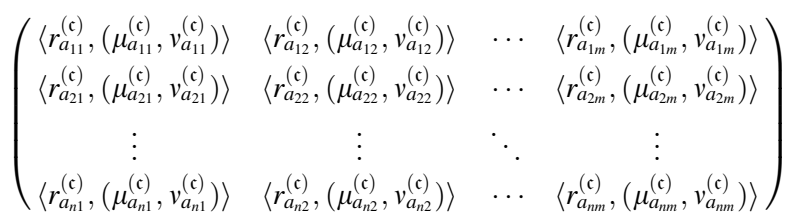

- Step 2. For MAGDM, the individual opinions of the decision-makers are organized to have a generic opinion of all experts about an alternative related to the attributes. This directs to the formation of aggregated $\mathrm{CFFNS}_{f} \mathrm{DM} \quad\left(\mathrm{ACFFNS}_{f} \mathrm{DM}\right) \quad \mathbf{5}=$ $(\mathbf{6 5})_{n \times m}$. The $\mathrm{CFFNS}_{f}$ DM of all experts are assembled with the help of $\mathrm{CFFNS}_{f}$ weighted average $\left(\mathrm{CFFNS}_{f} \mathrm{WA}\right)$ operator as follows:

$$
\begin{aligned}
& \mathfrak{G}_{g t}=C F F N S_{f} W A_{\zeta}\left(\mathfrak{G}_{g t}^{(1)}, \boldsymbol{G}_{g t}^{(2)}, \ldots, \mathfrak{G}_{g t}^{(l)}\right) \\
& =\zeta_{1} \mathfrak{G}_{g t}^{(1)} \oplus \zeta_{2} \mathfrak{G}_{g t}^{(2)} \oplus \ldots \oplus \zeta_{l} \tilde{\mathfrak{G}}_{g t}^{(l)}
\end{aligned}
$$

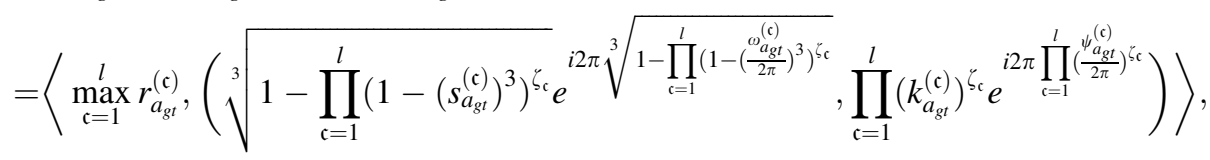

where $\mathfrak{c}=\{1,2, \ldots, l\}$.

Each entry of the $\mathrm{CFFNS}_{f} \mathrm{DM}$ has the form $\boldsymbol{(}_{g t}^{(\mathfrak{c})}=$ $\left\langle r_{a_{g t}}^{(\mathfrak{c})},\left(\mu_{a_{g t}}^{(\mathfrak{c})}, v_{a_{g t}}^{(\mathrm{c})}\right)\right\rangle=\left\langle r_{a_{g t}}^{(\mathfrak{c})},\left(s_{a_{g t}}^{(\mathfrak{c})} e^{i \omega_{a_{g t}}^{(\mathrm{c})}}, k_{a_{g t}}^{(\mathfrak{c})} e^{i \psi_{a_{g t}}^{(\mathrm{c})}}\right)\right\rangle$. where $\quad \boldsymbol{\sigma}_{g t}=\left\langle r_{a_{g t}},\left(\mu_{a_{g t}}, v_{a_{g t}}\right)\right\rangle=\left\langle r_{a_{g t}},\left(s_{a_{g t}} e^{i \omega_{a_{g t}}}\right.\right.$, $\left.\left.k_{a_{g t}} e^{i \psi_{a_{g t}}}\right)\right\rangle, g=1,2,3, \ldots, n, \quad$ and $t=1,2,3, \ldots, m$. The $\mathrm{ACFFNS}_{f} \mathrm{DM}$ can be form as follows: 


$$
\begin{aligned}
& \mathfrak{G}= \\
& \left(\begin{array}{cccc}
\left\langle r_{a_{11}},\left(\mu_{a_{11}}, v_{a_{11}}\right)\right\rangle & \left\langle r_{a_{12}},\left(\mu_{a_{12}}, v_{a_{12}}\right)\right\rangle & \ldots & \left\langle r_{a_{1 m}},\left(\mu_{a_{1 m}}, v_{a_{1 m}}\right)\right\rangle \\
\left\langle r_{a_{21}},\left(\mu_{a_{21}}, v_{a_{21}}\right)\right\rangle & \left\langle r_{a_{22}},\left(\mu_{a_{22}}, v_{a_{22}}\right)\right\rangle & \ldots & \left\langle r_{a_{2 m}},\left(\mu_{a_{2 m}}, v_{a_{2 m}}\right)\right\rangle \\
\vdots & \vdots & \ddots & \vdots \\
\left\langle r_{a_{n 1}},\left(\mu_{a_{n 1}}, v_{a_{n 1}}\right)\right\rangle & \left\langle r_{a_{n 2}},\left(\mu_{a_{n 2}}, v_{a_{n 2}}\right)\right\rangle & \cdots & \left\langle r_{a_{m m}},\left(\mu_{a_{n m}}, v_{a_{m m}}\right)\right\rangle
\end{array}\right)
\end{aligned}
$$

- Step 3. The attributes nominated by experts may not be equally important and valuable in a MAGDM problem. Therefore, each decision-maker ranks these attributes and assigns a CFFNS $_{f}$ weightage according to the grading criteria defined by the experts. Let $\kappa_{t}^{(\mathrm{c})}=\left\langle r_{a_{t}}^{(\mathrm{c})},\left(\mu_{a_{t}}^{(\mathrm{c})}, v_{a_{t}}^{(\mathrm{c})}\right)\right\rangle=\left\langle r_{a_{t}}^{(\mathrm{c})},\left(s_{a_{t}}^{(\mathrm{c})} e^{i \omega_{a_{t}}^{(\mathrm{c})}}, k_{a_{t}}^{(\mathrm{c})} e^{i \psi_{a_{t}}^{(\mathrm{c})}}\right\rangle\right.$ be the CFFNS $_{f}$ weight assigned by decision-maker $\mathcal{D}_{\mathfrak{c}}$ to the attribute $\mathfrak{B}_{t}$. To compute the $\mathrm{CFFNS}_{f}$ weight vector $\kappa=\left(\kappa_{1}, \kappa_{2}, \kappa_{3}, \ldots, \kappa_{m}\right)^{T}$ of attributes, the $\mathrm{CFFNS}_{f} \mathrm{Ns}$ corresponding to the grade values, assigned by decision-makers are aggregated as follows:
The $\mathrm{AWCFFNS}_{f} \mathrm{DM}$ is constructed as follows: $\widehat{\mathfrak{G}}=$

$$
\left(\begin{array}{cccc}
\left\langle\hat{r}_{a_{11}},\left(\hat{\mu}_{a_{11}}, \hat{v}_{a_{11}}\right)\right\rangle & \left\langle\hat{r}_{a_{12}},\left(\hat{\mu}_{a_{12}}, \hat{v}_{a_{12}}\right)\right\rangle & \cdots & \left\langle\hat{r}_{a_{1 m}},\left(\hat{\mu}_{a_{1 m}}, \hat{v}_{a_{1 m}}\right)\right\rangle \\
\left\langle\hat{r}_{a_{21}},\left(\hat{\mu}_{a_{21}}, \hat{v}_{a_{21}}\right)\right\rangle & \left\langle\hat{r}_{a_{22} 2},\left(\hat{\mu}_{a_{22} 2}, \hat{v}_{a_{22}}\right)\right\rangle & \cdots & \left\langle\hat{r}_{a_{2 m}},\left(\hat{\mu}_{a_{2 m}}, \hat{v}_{a_{2 m}}\right)\right\rangle \\
\vdots & \vdots & \ddots & \vdots \\
\left\langle\hat{r}_{a_{n 1}},\left(\hat{\mu}_{a_{n 1}}, \hat{v}_{a_{n 1}}\right)\right\rangle & \left\langle\hat{r}_{a_{n 2}},\left(\hat{\mu}_{a_{n 2}}, \hat{v}_{a_{n 2}}\right)\right\rangle & \cdots & \left\langle\hat{r}_{a_{m m}},\left(\hat{\mu}_{a_{m m}}, \hat{v}_{a_{m n}}\right)\right\rangle
\end{array}\right)
$$

- Step 5. Let $\mathfrak{B}^{-}$and $\mathfrak{B}^{+}$represent the collection of costtype and benefit-type attributes, respectively. Then $\mathrm{CFFNS}_{f}$ positive ideal solution $\left(\mathrm{CFFNS}_{f}\right.$-PIS) $\widetilde{\mathfrak{b}}_{t}=$ $\left\langle\tilde{r}_{a_{t}},\left(\tilde{\mu}_{a_{t}}, \tilde{v}_{a_{t}}\right)\right\rangle$ related to attribute $\mathfrak{B}_{t}$ can be chosen as follows:

$$
\widetilde{\mathfrak{G}}_{t}= \begin{cases}\max _{1 \leq g \leq n} \widehat{\mathfrak{G}}_{g t}, & \text { if } \mathfrak{B}_{t} \in \mathfrak{B}^{+}, \\ \min _{1 \leq g \leq n} \widehat{\mathfrak{G}}_{g t}, & \text { if } \mathfrak{B}_{t} \in \mathfrak{B}^{-} .\end{cases}
$$

$$
\begin{aligned}
& \kappa_{t}=C F F N S_{f} W A_{\zeta}\left(\kappa_{t}^{(1)}, \kappa_{t}^{(2)}, \ldots, \kappa_{t}^{(l)}\right) \\
& =\zeta_{1} \kappa_{t}^{(1)} \oplus \zeta_{2} \kappa_{t}^{(2)} \oplus \ldots \oplus \zeta_{l} \kappa_{t}^{(l)} \\
& =\left\langle\max _{c=1}^{l} r_{a_{t}}^{(c)},\left(\sqrt[3]{1-\prod_{c=1}^{l}\left(1-\left(s_{a_{t}}^{(c)}\right)^{3}\right)^{\zeta c}} e^{i 2 \pi \sqrt[3]{1-\prod_{c=1}^{l}\left(1-\left(\frac{w_{a t}^{(c)}}{2 \pi}\right)^{3}\right)^{\zeta_{c}}}}, \prod_{c=1}^{l}\left(k_{a_{t}}^{(c)}\right)^{\zeta c} e^{i 2 \pi \prod_{c=1}^{l}\left(\frac{\psi_{a t}^{(c)}}{2 \pi}\right)^{\zeta_{c}}}\right)\right\rangle, \\
& =\left\langle r_{a_{t}},\left(\mu_{a_{t}}, v_{a_{t}}\right)\right\rangle \text {, } \\
& =\left\langle r_{a_{t}},\left(s_{a_{t}} e^{i \omega_{a_{t}}}, k_{a_{t}} e^{i \psi_{a_{t}}}\right)\right\rangle,
\end{aligned}
$$

where $t=1,2,3, \ldots, m$.

- Step 4. Construct the aggregated weighted $\mathrm{CFFNS}_{f} \mathrm{DM}$ $\left(\mathrm{AWCFFNS}_{f} \mathrm{DM}\right) \widehat{\mathfrak{F}}=\left(\widehat{\mathfrak{F}}_{g t}\right)_{n \times m}$ by using $\mathrm{CFFNS}_{f} \mathrm{DM}$ $\left(\mathfrak{5}\right.$ and the weight vector $\kappa_{t}$ of attributes, as follows:
The $\mathrm{CFFNS}_{f}$ negative ideal solution $\left(\mathrm{CFFNS}_{f}\right.$-NIS) $\breve{\mathfrak{G}}_{t}=\left\langle\breve{r}_{a_{t}},\left(\breve{\mu}_{a_{t}}, \breve{v}_{a_{t}}\right)\right\rangle$ with respect to the attribute $\mathfrak{B}_{t}$ can be determined as follows:

$$
\begin{aligned}
& \widehat{\mathfrak{G}}_{g t}=\mathfrak{G}_{g t} \otimes \kappa_{t} \\
& =\left\langle\min \left(r_{a_{g t}}, r_{a_{t}}\right),\left(s_{a_{g t}} s_{a_{t}} e^{i 2 \pi\left(\frac{\omega a_{g t}}{2 \pi}\right)\left(\frac{\omega_{a t}}{2 \pi}\right)}, \sqrt[3]{\left(k_{a_{g t}}\right)^{3}+\left(k_{a_{t}}\right)^{3}-\left(k_{a_{g t}}\right)^{3}\left(k_{a_{t}}\right)^{3}}\right.\right.
\end{aligned}
$$

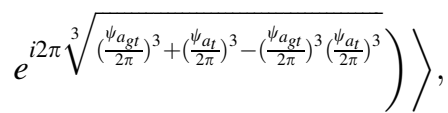

$$
\begin{aligned}
& =\left\langle\hat{r}_{a_{g t}},\left(\hat{\mu}_{a_{g t}}, \hat{v}_{a_{g t}}\right)\right\rangle \text {, } \\
& =\left\langle\hat{r}_{a_{g t}},\left(\hat{s}_{a_{g t} t} e^{i \hat{\omega}_{a t}}, \hat{k}_{a_{g t}} e^{i \hat{\psi}_{a_{g t}}}\right)\right\rangle \text {. }
\end{aligned}
$$




$$
\breve{\mathfrak{G}}_{t}= \begin{cases}\min _{1 \leq g \leq n} \widehat{\mathfrak{G}}_{g t}, & \text { if } \mathfrak{B}_{t} \in \mathfrak{B}^{+}, \\ \max _{1 \leq g \leq n} \widehat{\mathfrak{G}}_{g t}, & \text { if } \mathfrak{B}_{t} \in \mathfrak{B}^{-} .\end{cases}
$$

The $\mathrm{CFFNS}_{f} \mathrm{Ns}$ are compared on the basis of accuracy function and score function to obtain $\mathrm{CFFNS}_{f}$-PIS and CFFNS $_{f}$-NIS.

- Step 6. Now, to find the optimal alternative which is away from $\mathrm{CFFNS}_{f}$-NIS and closest to $\mathrm{CFFNS}_{f}$-PIS, we evaluate the distance of each alternative $\mathfrak{I}_{g}$ from $\mathrm{CFFNS}_{f}$-PIS and $\mathrm{CFFNS}_{f}$-NIS. The distance between any of the alternative and $\mathrm{CFFNS}_{f}$-PIS can be calculated as follows:

$$
\begin{aligned}
& d\left(\widetilde{\mathfrak{I}}_{g}, \widetilde{\mathfrak{W}}_{t}\right)= \\
& \sum_{t=1}^{m} \sqrt{\frac{1}{3}\left\{\left(\frac{\hat{r}_{a_{g t}}}{N-1}-\frac{\tilde{r}_{a_{t}}}{N-1}\right)^{2}+\left(\hat{s}_{a_{g t}}^{3}-\hat{s}_{a_{t}}^{3}\right)^{2}+\left(\hat{k}_{a_{g t}}^{3}-\hat{k}_{a_{t}}^{3}\right)^{2}+\frac{1}{64 \pi^{6}}\right.} \\
& \sqrt{\left.\left(\left(\hat{\omega}_{a_{g t}}{ }^{3}-\tilde{\omega}_{a_{t}}{ }^{3}\right)^{2}+\left(\hat{\psi}_{a_{g t}}{ }^{3}-\tilde{\psi}_{a_{t}}{ }^{3}\right)^{2}\right)\right\}}
\end{aligned}
$$

Similarly, the distance between any of the alternative and CFFNS $_{f}$-NIS can be calculated as follows:

$$
\begin{aligned}
& d\left(\mathfrak{\Im}_{g}, \breve{\mathfrak{G}}_{t}\right)= \\
& \sum_{t=1}^{m} \sqrt{\frac{1}{3}\left\{\left(\frac{\hat{r}_{a_{g t}}}{N-1}-\frac{\breve{r}_{a_{t}}}{N-1}\right)^{2}+\left(\hat{s}_{a_{g t}}^{3}-\breve{s}_{a_{t}}^{3}\right)^{2}+\left(\hat{k}_{a_{s t}}^{3}-{\breve{a_{t}}}_{a_{t}}^{3}\right)^{2}+\frac{1}{64 \pi^{6}}\right.} \\
& \sqrt{\left.\left(\left(\hat{\omega}_{a_{g t}}^{3}-\breve{\omega}_{a_{t}}^{3}\right)^{2}+\left(\hat{\psi}_{a_{g t}}^{3}-\breve{\psi}_{a_{t}}^{3}\right)^{2}\right)\right\}}
\end{aligned}
$$

- Step 7. To find the most suitable alternative, we compare the alternative by some ranking index. The revised closeness index (Vencheh and Mirjaberi 2014) corresponding to the alternative $\mathfrak{I}_{g}$ can be evaluated by utilizing the formula:

$$
\Psi\left(\mathfrak{I}_{g}\right)=\frac{d\left(\mathfrak{I}_{g}, \breve{\mathfrak{G}}_{t}\right)}{d_{\max }\left(\widetilde{\mathfrak{I}}_{g}, \breve{\mathfrak{G}}_{t}\right)}-\frac{d\left(\mathfrak{I}_{g}, \widetilde{\boldsymbol{G}}_{t}\right)}{d_{\min }\left(\mathfrak{I}_{g}, \widetilde{\mathfrak{G}}_{t}\right)},
$$

where $g=1,2,3, \ldots, n$, and

$$
\begin{aligned}
& d_{\max }\left(\mathfrak{I}_{g}, \breve{\mathfrak{W}}_{t}\right)=\max _{1 \leq g \leq n}\left(\mathfrak{I}_{g}, \breve{\mathfrak{G}}_{t}\right), \\
& d_{\min }\left(\mathfrak{I}_{g}, \breve{\mathfrak{G}}_{t}\right)=\min _{1 \leq g \leq n}\left(\mathfrak{I}_{g}, \widetilde{\mathfrak{G}}_{t}\right) .
\end{aligned}
$$

- Step 8. After the evaluated results of closeness index, the alternatives are arranged in an ascending order with respect to revised closeness index. The alternative having maximum value of closeness index will be the optimal solution of MAGDM problem.

The general steps of $\mathrm{CFFNS}_{f}$-TOPSIS method are summarized in Figure 2.

\section{Selection of the most suitable city in the USA for farming}

Suppose that an investor $X$ wants to purchase land for agriculture farming in a favorable city of the USA. For this purpose, he arranged a panel of four decision-makers $\mathfrak{D}_{1}, \mathfrak{D}_{2}, \mathfrak{D}_{3}$ and $\mathfrak{D}_{4}$ to thoroughly judge the essential needs of the best land for farming. Since, each decision-maker has his own importance and opinions, so, $\zeta=$ $(0.240 .350 .230 .18)^{T}$ represents the weightage of experts in the decision-making panel. Clearly, $\sum_{c=1}^{4} \zeta_{c}=1$. The following cities are under consideration as alternatives for this problem:

- $\mathfrak{I}_{1}$ : Boston, Massachusetts

- $\mathfrak{I}_{2}$ : Portland, Oregon

- $\mathfrak{I}_{3}$ : New York city, New York

- $\mathfrak{I}_{4}$ : Minneapolis, Minnesota

After discussion, all decision-makers identify the following factors as the attributes for this MAGDM problem:

- $\mathfrak{B}_{1}$ : Initial cost

- $\mathfrak{B}_{2}$ : Environmental destruction

- $\mathfrak{B}_{3}$ : Topography

- $\mathfrak{B}_{4}$ : Climate of the Area

- $\mathfrak{B}_{5}$ : Maintenance cost

- $\mathfrak{B}_{6}$ : Soil

- $\mathfrak{B}_{7}$ : Water quality \& availability

The stepwise solution of this MAGDM problem by following $\mathrm{CFFNS}_{f}$-TOPSIS method is given as follows:

- Step 1. According to the above-mentioned attributes, each expert assesses the alternatives regarding all attributes using 5-soft, given in Table 42, where:

-

- DOO represent 'Good',

- DO represent 'Average',

- $\bigcirc$ represents 'Satisfactory',

- $\diamond$ represents 'Below average.

The experts $\mathfrak{D}_{1}, \mathfrak{D}_{2}, \mathfrak{D}_{3}$ and $\mathfrak{D}_{4}$ will use the grading criteria given by Table 5 , to assign the $\mathrm{CFFNS}_{f} \mathrm{~N}$ corresponding to each rank. The individual $\mathrm{CFFNS}_{f} \mathrm{DMs}$ of the experts are arranged by Tables $43-46$. 
Fig. 2 Flow chart of $\mathrm{CFFNS}_{f}$ TOPSIS method

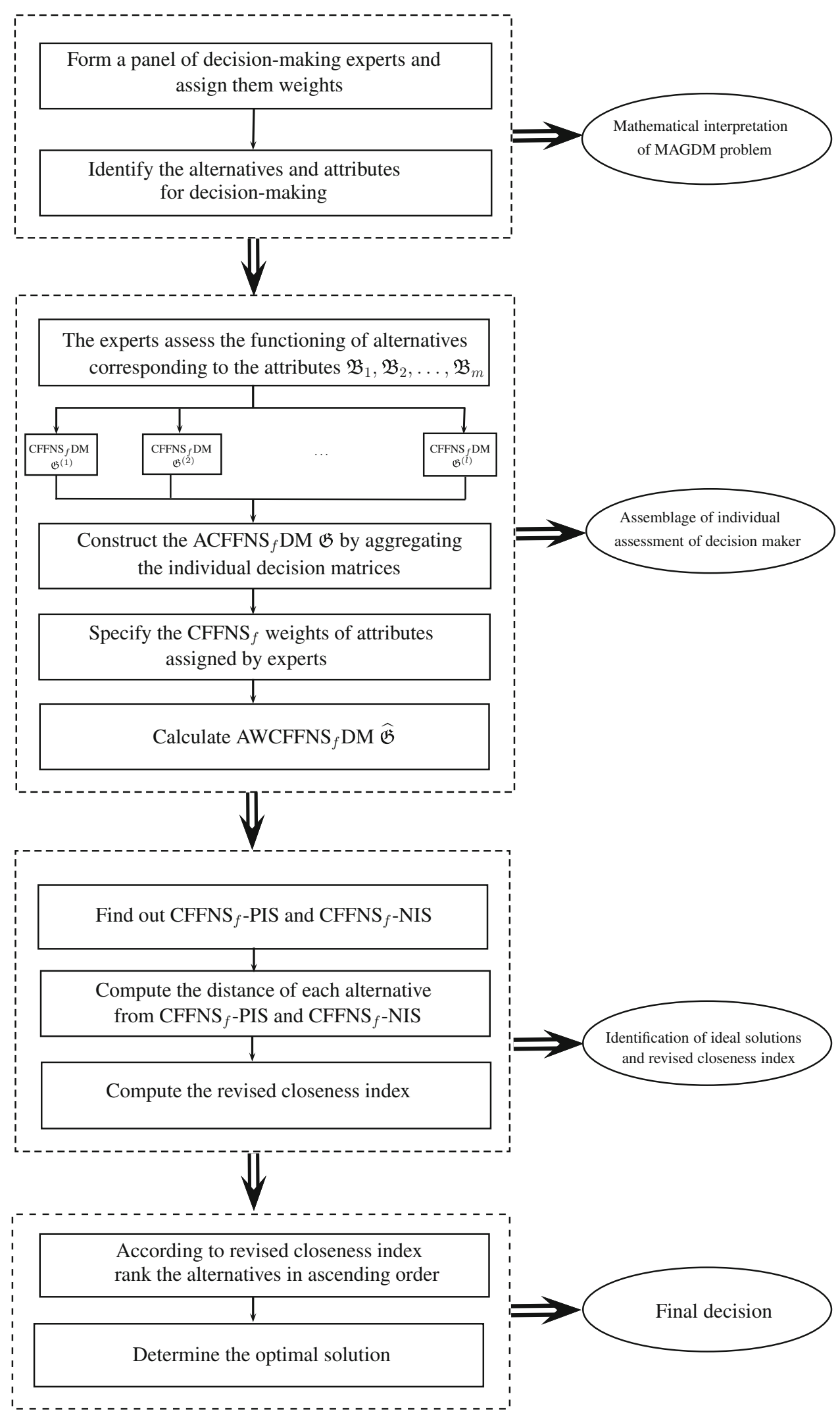


Table 42 Expert's assessment of alternatives corresponding to each attribute

\begin{tabular}{|c|c|c|c|c|c|}
\hline Attributes & Alternatives & $\mathfrak{D}_{1}$ & $\mathfrak{D}_{2}$ & $\mathfrak{D}_{3}$ & $\mathfrak{D}_{4}$ \\
\hline \multirow[t]{4}{*}{$\mathfrak{B}_{1}$} & $\mathfrak{I}_{1}$ & $\varnothing=1$ & $\varnothing=1$ & $\varnothing \nabla=2$ & $\nabla D=2$ \\
\hline & $\mathfrak{I}_{2}$ & $D D=2$ & $\varnothing \nabla \infty=3$ & $\triangleright \nabla \varnothing=3$ & $\varnothing \Delta \nabla=3$ \\
\hline & $\mathfrak{J}_{3}$ & $\infty \Delta \infty=3$ & வ০০০ $=4$ & D০০০ $=4$ & ऽ০০০ $=4$ \\
\hline & $\mathfrak{I}_{4}$ & $\nabla D=2$ & $\nabla \nabla=2$ & $\nabla \nabla=2$ & $\nabla \nabla=2$ \\
\hline \multirow[t]{4}{*}{$\mathfrak{B}_{2}$} & $\mathfrak{J}_{1}$ & $\varnothing \nabla=2$ & $\varnothing \nabla=2$ & $\nabla \nabla=2$ & $\nabla \nabla=2$ \\
\hline & $\mathfrak{I}_{2}$ & $\triangle D=2$ & $\odot=1$ & $\nabla D=2$ & $\varnothing \nabla=2$ \\
\hline & $\mathfrak{J}_{3}$ & $\nabla \nabla=2$ & $\nabla \nabla=2$ & $\nabla \nabla=2$ & $\varnothing \nabla \infty=3$ \\
\hline & $\mathfrak{I}_{4}$ & $\triangle D=2$ & $\varnothing \nabla=2$ & $\triangle \nabla=2$ & $\triangle \nabla D=3$ \\
\hline \multirow[t]{4}{*}{$\mathfrak{B}_{3}$} & $\mathfrak{I}_{1}$ & $\triangle D D=3$ & $\triangle \otimes D=3$ & $\triangle \triangleright \triangleright=3$ & ০০০০ $=4$ \\
\hline & $\mathfrak{I}_{2}$ & $\nabla D=2$ & $\varnothing \nabla=2$ & $\varnothing \nabla=2$ & $\nabla D=2$ \\
\hline & $\mathfrak{J}_{3}$ & $\varnothing=1$ & $\varnothing=1$ & $\diamond=0$ & $\diamond=0$ \\
\hline & $\mathfrak{I}_{4}$ & $\varnothing \nabla=2$ & $\varnothing \nabla=2$ & $\varnothing \nabla=2$ & $\varnothing \nabla=2$ \\
\hline \multirow[t]{4}{*}{$\mathfrak{B}_{4}$} & $\mathfrak{I}_{1}$ & $\triangle \nabla=2$ & $\nabla \nabla=2$ & $\varnothing=1$ & $\varnothing=1$ \\
\hline & $\mathfrak{I}_{2}$ & $\triangle D D=3$ & $\triangle D D=3$ & $\triangle D \triangle=3$ & ৪০০০ $=4$ \\
\hline & $\mathfrak{J}_{3}$ & $\triangle D D=3$ & D০০০ $=4$ & ৩০০০ $=4$ & $\triangle D D=3$ \\
\hline & $\mathfrak{I}_{4}$ & $000=3$ & $000=3$ & $\nabla \nabla \nabla=3$ & ऽ০০০ $=4$ \\
\hline \multirow[t]{4}{*}{$\mathfrak{B}_{5}$} & $\mathfrak{I}_{1}$ & $\triangle D D=3$ & D০০০ $=4$ & ৩০০০ $=4$ & வ০০০ $=4$ \\
\hline & $\mathfrak{I}_{2}$ & $\nabla D=2$ & $\triangle \nabla D=3$ & $\nabla \nabla=2$ & $\triangle \otimes D=3$ \\
\hline & $\mathfrak{I}_{3}$ & $\diamond=0$ & $\diamond=0$ & $\diamond=0$ & $\diamond=0$ \\
\hline & $\mathfrak{I}_{4}$ & $\triangle D D=3$ & $\triangle \nabla D=3$ & $\triangle \otimes D=3$ & $\nabla D=2$ \\
\hline \multirow[t]{4}{*}{$\mathfrak{B}_{6}$} & $\mathfrak{I}_{1}$ & $\triangle D D=3$ & $\triangle \triangleright \triangleright=3$ & ๑০০০ $=4$ & $\triangle \triangleright \triangleright=3$ \\
\hline & $\mathfrak{I}_{2}$ & $\nabla \nu=2$ & $\varnothing=1$ & $\nabla \nabla=2$ & $\varnothing=1$ \\
\hline & $\mathfrak{I}_{3}$ & $\nabla D=2$ & $\varnothing=1$ & $\varnothing=1$ & $\nabla \nabla=2$ \\
\hline & $\mathfrak{I}_{4}$ & $000=3$ & $\triangleright \triangleright=2$ & $\triangleright \triangleright=2$ & $\nabla D=2$ \\
\hline \multirow[t]{4}{*}{$\mathfrak{B}_{7}$} & $\mathfrak{I}_{1}$ & D০DO $=4$ & வ০০০ $=4$ & ऽ০০০ $=4$ & வ০০০ $=4$ \\
\hline & $\mathfrak{I}_{2}$ & $\nabla D=2$ & $\nabla \nabla=2$ & $\triangle \nabla D=3$ & $\nabla \nabla=2$ \\
\hline & $\mathfrak{I}_{3}$ & $\diamond=0$ & $\diamond=0$ & $\odot=1$ & $\varnothing=1$ \\
\hline & $\mathfrak{T}_{4}$ & $\triangle \nabla \nu=3$ & $\triangle \nabla \nu=3$ & ৩৭০০ $=4$ & வ০০০ $=4$ \\
\hline
\end{tabular}

Table 43 Tabulated form of $\mathrm{CFFNS}_{f} \mathrm{DM} \mathfrak{G}^{(1)}$ of expert $\mathfrak{D}_{1}$

\begin{tabular}{|c|c|c|c|}
\hline$\left(\mathcal{H}^{(1)}, \mathcal{Q}, 5\right)$ & $\mathfrak{B}_{1}$ & $\mathfrak{B}_{2}$ & $\mathfrak{B}_{3}$ \\
\hline $\begin{array}{l}\mathfrak{I}_{1} \\
\mathfrak{I}_{2} \\
\mathfrak{I}_{3} \\
\mathfrak{I}_{4}\end{array}$ & $\begin{array}{l}\left\langle 1,\left(0.38 e^{i 0.78 \pi}, 0.73 e^{i 1.37 \pi}\right)\right\rangle \\
\left\langle 2,\left(0.41 e^{i 0.98 \pi}, 0.52 e^{i 1.12 \pi}\right)\right\rangle \\
\left\langle 3,\left(0.81 e^{i 1.51 \pi}, 0.38 e^{i 0.68 \pi}\right)\right\rangle \\
\left\langle 2,\left(0.53 e^{i 1.01 \pi}, 0.44 e^{i 0.82 \pi}\right)\right\rangle\end{array}$ & $\begin{array}{l}\left\langle 2,\left(0.42 e^{i 1.23 \pi}, 0.51 e^{i 0.96 \pi}\right)\right\rangle \\
\left\langle 2,\left(0.56 e^{i 0.87 \pi}, 0.45 e^{i 1.11 \pi}\right)\right\rangle \\
\left\langle 2,\left(0.44 e^{i 1.25 \pi}, 0.53 e^{i 0.94 \pi}\right)\right\rangle \\
\left\langle 2,\left(0.52 e^{i 0.93 \pi}, 0.41 e^{i 1.12 \pi}\right)\right\rangle\end{array}$ & $\begin{array}{l}\left\langle 3,\left(0.68 e^{i 1.61 \pi}, 0.31 e^{i 0.67 \pi}\right)\right\rangle \\
\left\langle 2,\left(0.64 e^{i 1.21 \pi}, 0.56 e^{i 0.92 \pi}\right)\right\rangle \\
\left\langle 1,\left(0.33 e^{i 0.55 \pi}, 0.81 e^{i 1.35 \pi}\right)\right\rangle \\
\left\langle 2,\left(0.44 e^{i 1.19 \pi}, 0.61 e^{i 0.90 \pi}\right)\right\rangle\end{array}$ \\
\hline & $\mathfrak{B}_{4}$ & $\mathfrak{B}_{5}$ & $\mathfrak{B}_{6}$ \\
\hline $\begin{array}{l}\mathfrak{I}_{1} \\
\mathfrak{I}_{2} \\
\mathfrak{I}_{3} \\
\mathfrak{I}_{4}\end{array}$ & $\begin{array}{l}\left\langle 2,\left(0.45 e^{i 1.14 \pi}, 0.59 e^{i 1.23 \pi}\right)\right\rangle \\
\left\langle 3,\left(0.67 e^{i 1.51 \pi}, 0.35 e^{i 0.42 \pi}\right)\right\rangle \\
\left\langle 3,\left(0.78 e^{i 1.37 \pi}, 0.24 e^{i 0.54 \pi}\right)\right\rangle \\
\left\langle 3,\left(0.68 e^{i 1.49 \pi}, 0.32 e^{i 0.47 \pi}\right)\right\rangle\end{array}$ & $\begin{array}{l}\left\langle 3,\left(0.74 e^{i 1.36 \pi}, 0.26 e^{i 0.61 \pi}\right)\right\rangle \\
\left\langle 2,\left(0.59 e^{i 1.12 \pi}, 0.54 e^{i 1.21 \pi}\right)\right\rangle \\
\left\langle 0,\left(0.07 e^{i 0.18 \pi}, 0.92 e^{i 1.77 \pi}\right)\right\rangle \\
\left\langle 3,\left(0.77 e^{i 1.49 \pi}, 0.34 e^{i 0.52 \pi}\right)\right\rangle\end{array}$ & $\begin{array}{l}\left\langle 3,\left(0.80 e^{i 1.34 \pi}, 0.33 e^{i 0.59 \pi}\right)\right\rangle \\
\left\langle 2,\left(0.61 e^{i 1.13 \pi}, 0.48 e^{i 0.87 \pi}\right)\right\rangle \\
\left\langle 2,\left(0.50 e^{i 0.95 \pi}, 0.54 e^{i 1.25 \pi}\right)\right\rangle \\
\left\langle 3,\left(0.71 e^{i 1.43 \pi}, 0.24 e^{i 0.76 \pi}\right)\right\rangle\end{array}$ \\
\hline \multicolumn{4}{|c|}{$\mathfrak{B}_{7}$} \\
\hline $\begin{array}{l}\mathfrak{I}_{1} \\
\mathfrak{I}_{2} \\
\mathfrak{I}_{3} \\
\mathfrak{I}_{4}\end{array}$ & $\begin{array}{l}\left\langle 4,\left(0.93 e^{i 1.93 \pi}, 0.07 e^{i 0.32 \pi}\right)\right\rangle \\
\left\langle 2,\left(0.47 e^{i 1.23 \pi}, 0.62 e^{i 0.98 \pi}\right)\right\rangle \\
\left\langle 0,\left(0.16 e^{i 0.32 \pi}, 0.86 e^{i 1.91 \pi}\right)\right\rangle \\
\left\langle 3,\left(0.69 e^{i 1.33 \pi}, 0.31 e^{i 0.58 \pi}\right)\right\rangle\end{array}$ & & \\
\hline
\end{tabular}


Table 44 Tabulated form of $\mathrm{CFFNS}_{f} \mathrm{DM} \boldsymbol{5}^{(2)}$ of expert $\mathfrak{D}_{2}$

\begin{tabular}{|c|c|c|c|}
\hline$\left(\mathcal{H}^{(2)}, \mathcal{Q}, 5\right)$ & $\mathfrak{B}_{1}$ & $\mathfrak{B}_{2}$ & $\mathfrak{B}_{3}$ \\
\hline $\begin{array}{l}\mathfrak{I}_{1} \\
\mathfrak{I}_{2} \\
\mathfrak{I}_{3} \\
\mathfrak{I}_{4}\end{array}$ & $\begin{array}{l}\left\langle 1,\left(0.29 e^{i 0.45 \pi}, 0.69 e^{i 1.67 \pi}\right)\right\rangle \\
\left\langle 3,\left(0.69 e^{i 1.62 \pi}, 0.33 e^{i 0.57 \pi}\right)\right\rangle \\
\left\langle 4,\left(0.97 e^{i 1.93 \pi}, 0.03 e^{i 0.07 \pi}\right)\right\rangle \\
\left\langle 2,\left(0.51 e^{i 1.11 \pi}, 0.42 e^{i 1.21 \pi}\right)\right\rangle\end{array}$ & $\begin{array}{l}\left\langle 2,\left(0.54 e^{i 0.88 \pi}, 0.43 e^{i 1.24 \pi}\right)\right\rangle \\
\left\langle 1,\left(0.32 e^{i 0.51 \pi}, 0.82 e^{i 1.59 \pi}\right)\right\rangle \\
\left\langle 2,\left(0.58 e^{i 1.25 \pi}, 0.47 e^{i 0.98 \pi}\right)\right\rangle \\
\left\langle 2,\left(0.46 e^{i 0.96 \pi}, 0.55 e^{i 1.23 \pi}\right)\right\rangle\end{array}$ & $\begin{array}{l}\left\langle 3,\left(0.78 e^{i 1.54 \pi}, 0.34 e^{i 0.57 \pi}\right)\right\rangle \\
\left\langle 2,\left(0.63 e^{i 1.17 \pi}, 0.57 e^{i 0.88 \pi}\right)\right\rangle \\
\left\langle 1,\left(0.23 e^{i 0.61 \pi}, 0.72 e^{i 1.47 \pi}\right)\right\rangle \\
\left\langle 2,\left(0.43 e^{i 1.15 \pi}, 0.62 e^{i 0.86 \pi}\right)\right\rangle\end{array}$ \\
\hline & $\mathfrak{B}_{4}$ & $\mathfrak{B}_{5}$ & $\mathfrak{B}_{6}$ \\
\hline $\begin{array}{l}\mathfrak{I}_{1} \\
\mathfrak{I}_{2} \\
\mathfrak{I}_{3} \\
\mathfrak{I}_{4}\end{array}$ & $\begin{array}{l}\left\langle 2,\left(0.60 e^{i 1.16 \pi}, 0.55 e^{i 1.25 \pi}\right)\right\rangle \\
\left\langle 3,\left(0.79 e^{i 1.39 \pi}, 0.38 e^{i 0.45 \pi}\right)\right\rangle \\
\left\langle 4,\left(0.86 e^{i 1.85 \pi}, 0.03 e^{i 0.17 \pi}\right)\right\rangle \\
\left\langle 3,\left(0.69 e^{i 1.48 \pi}, 0.22 e^{i 0.56 \pi}\right)\right\rangle\end{array}$ & $\begin{array}{l}\left\langle 4,\left(0.97 e^{i 1.89 \pi}, 0.03 e^{i 0.09 \pi}\right)\right\rangle \\
\left\langle 3,\left(0.76 e^{i 1.37 \pi}, 0.31 e^{i 0.72 \pi}\right)\right\rangle \\
\left\langle 0,\left(0.13 e^{i 0.29 \pi}, 0.88 e^{i 1.85 \pi}\right)\right\rangle \\
\left\langle 3,\left(0.75 e^{i 1.47 \pi}, 0.29 e^{i 0.48 \pi}\right)\right\rangle\end{array}$ & $\begin{array}{l}\left\langle 3,\left(0.82 e^{i 1.39 \pi}, 0.26 e^{i 0.73 \pi}\right)\right\rangle \\
\left\langle 1,\left(0.37 e^{i 0.73 \pi}, 0.79 e^{i 1.39 \pi}\right)\right\rangle \\
\left\langle 1,\left(0.25 e^{i 0.67 \pi}, 0.71 e^{i 1.47 \pi}\right)\right\rangle \\
\left\langle 2,\left(0.49 e^{i 0.85 \pi}, 0.55 e^{i 0.96 \pi}\right)\right\rangle\end{array}$ \\
\hline \multicolumn{4}{|c|}{$\mathfrak{B}_{7}$} \\
\hline $\begin{array}{l}\mathfrak{I}_{1} \\
\mathfrak{I}_{2} \\
\mathfrak{I}_{3} \\
\mathfrak{I}_{4}\end{array}$ & $\begin{array}{l}\left\langle 4,\left(0.92 e^{i 1.95 \pi}, 0.09 e^{i 0.31 \pi}\right)\right\rangle \\
\left\langle 2,\left(0.63 e^{i 0.83 \pi}, 0.55 e^{i 0.94 \pi}\right)\right\rangle \\
\left\langle 0,\left(0.02 e^{i 0.11 \pi}, 0.91 e^{i 1.88 \pi}\right)\right\rangle \\
\left\langle 3,\left(0.78 e^{i 1.41 \pi}, 0.31 e^{i 0.47 \pi}\right)\right\rangle\end{array}$ & & \\
\hline
\end{tabular}

Table 45 Tabulated form of $\mathrm{CFFNS}_{f} \mathrm{DM} \mathfrak{5}^{(3)}$ of expert $\mathfrak{D}_{3}$

\begin{tabular}{|c|c|c|c|}
\hline$\left(\mathcal{H}^{(3)}, \mathcal{Q}, 5\right)$ & $\mathfrak{B}_{1}$ & $\mathfrak{B}_{2}$ & $\mathfrak{B}_{3}$ \\
\hline $\begin{array}{l}\mathfrak{I}_{1} \\
\mathfrak{I}_{2} \\
\mathfrak{I}_{3} \\
\mathfrak{I}_{4}\end{array}$ & $\begin{array}{l}\left\langle 2,\left(0.43 e^{i 1.07 \pi}, 0.54 e^{i 0.83 \pi}\right)\right\rangle \\
\left\langle 3,\left(0.75 e^{i 1.48 \pi}, 0.24 e^{i 0.49 \pi}\right)\right\rangle \\
\left\langle 4,\left(0.89 e^{i 1.85 \pi}, 0.15 e^{i 0.28 \pi}\right)\right\rangle \\
\left\langle 2,\left(0.55 e^{i 1.13 \pi}, 0.46 e^{i 0.89 \pi}\right)\right\rangle\end{array}$ & $\begin{array}{l}\left\langle 2,\left(0.60 e^{i 1.27 \pi}, 0.49 e^{i 1.04 \pi}\right)\right\rangle \\
\left\langle 2,\left(0.48 e^{i 0.94 \pi}, 0.57 e^{i 1.09 \pi}\right)\right\rangle \\
\left\langle 2,\left(0.50 e^{i 1.29 \pi}, 0.59 e^{i 1.06 \pi}\right)\right\rangle \\
\left\langle 2,\left(0.62 e^{i 0.84 \pi}, 0.61 e^{i 1.24 \pi}\right)\right\rangle\end{array}$ & $\begin{array}{l}\left\langle 3,\left(0.81 e^{i 1.38 \pi}, 0.38 e^{i 0.61 \pi}\right)\right\rangle \\
\left\langle 2,\left(0.62 e^{i 1.13 \pi}, 0.58 e^{i 0.84 \pi}\right)\right\rangle \\
\left\langle 0,\left(0.02 e^{i 0.04 \pi}, 0.87 e^{i 1.75 \pi}\right)\right\rangle \\
\left\langle 2,\left(0.42 e^{i 1.11 \pi}, 0.63 e^{i 0.82 \pi}\right)\right\rangle\end{array}$ \\
\hline & $\mathfrak{B}_{4}$ & $\mathfrak{B}_{5}$ & $\mathfrak{B}_{6}$ \\
\hline $\begin{array}{l}\mathfrak{I}_{1} \\
\mathfrak{I}_{2} \\
\mathfrak{I}_{3} \\
\mathfrak{I}_{4}\end{array}$ & $\begin{array}{l}\left\langle 1,\left(0.24 e^{i 0.42 \pi}, 0.67 e^{i 1.39 \pi}\right)\right\rangle \\
\left\langle 3,\left(0.80 e^{i 1.37 \pi}, 0.28 e^{i 0.48 \pi}\right)\right\rangle \\
\left\langle 4,\left(0.92 e^{i 1.76 \pi}, 0.15 e^{i 0.28 \pi}\right)\right\rangle \\
\left\langle 3,\left(0.70 e^{i 1.45 \pi}, 0.37 e^{i 0.49 \pi}\right)\right\rangle\end{array}$ & $\begin{array}{l}\left\langle 4,\left(0.93 e^{i 1.77 \pi}, 0.04 e^{i 0.35 \pi}\right)\right\rangle \\
\left\langle 2,\left(0.46 e^{i 1.15 \pi}, 0.58 e^{i 0.82 \pi}\right)\right\rangle \\
\left\langle 0,\left(0.09 e^{i 0.12 \pi}, 0.89 e^{i 1.86 \pi}\right)\right\rangle \\
\left\langle 3,\left(0.84 e^{i 1.45 \pi}, 0.25 e^{i 0.63 \pi}\right)\right\rangle\end{array}$ & $\begin{array}{l}\left\langle 4,\left(0.88 e^{i 1.83 \pi}, 0.02 e^{i 0.31 \pi}\right)\right\rangle \\
\left\langle 2,\left(0.53 e^{i 0.82 \pi}, 0.47 e^{i 1.11 \pi}\right)\right\rangle \\
\left\langle 1,\left(0.34 e^{i 0.77 \pi}, 0.84 e^{i 1.58 \pi}\right)\right\rangle \\
\left\langle 2,\left(0.48 e^{i 0.97 \pi}, 0.56 e^{i 1.22 \pi}\right)\right\rangle\end{array}$ \\
\hline \multicolumn{4}{|c|}{$\mathfrak{B}_{7}$} \\
\hline $\begin{array}{l}\mathfrak{I}_{1} \\
\mathfrak{I}_{2} \\
\mathfrak{I}_{3} \\
\mathfrak{I}_{4}\end{array}$ & $\begin{array}{l}\left\langle 4,\left(0.86 e^{i 1.91 \pi}, 0.15 e^{i 0.29 \pi}\right)\right\rangle \\
\left\langle 3,\left(0.67 e^{i 1.32 \pi}, 0.23 e^{i 0.59 \pi}\right)\right\rangle \\
\left\langle 1,\left(0.27 e^{i 0.48 \pi}, 0.78 e^{i 1.51 \pi}\right)\right\rangle \\
\left\langle 4,\left(0.88 e^{i 1.89 \pi}, 0.13 e^{i 0.27 \pi}\right)\right\rangle\end{array}$ & & \\
\hline
\end{tabular}

- Step 2. The individual opinions of all decision-makers are assembled by employing the $\mathrm{CFFNS}_{f} \mathrm{WA}$ operator, as defined in Equation 2 and the results are accumulated in the $\mathrm{ACFFNS}_{f} \mathrm{DM}$ as shown in Table 47.

- Step 3. Experts associate $\mathrm{CFFNS}_{f} \mathrm{~N}$ to each attribute indicating the importance of that attribute in MAGDM problems which are summarized in Table 48. The $\mathrm{CFFNS}_{f}$ weight of each attribute is accumulated by CFFNS $_{f}$ WA operator defined in Equation 3 to form a $\mathrm{CFFNS}_{f}$ weight vector $\kappa$, given by:

$$
\kappa=\left(\begin{array}{c}
3,\left(0.731811 e^{i 1.527149 \pi}, 0.304928 e^{i 0.582725 \pi}\right) \\
4,\left(0.945072 e^{i 1.861182 \pi}, 0.054923 e^{i 0.236347 \pi}\right) \\
4,\left(0.860568 e^{i 1.651356 \pi}, 0.189314 e^{i 0.416769 \pi}\right) \\
3,\left(0.545443 e^{i 1.019597 \pi}, 0.613887 e^{i 1.185280 \pi}\right) \\
4,\left(0.826558 e^{i 1.645141 \pi}, 0.185730 e^{i 0.402961 \pi}\right) \\
3,\left(0.739877 e^{i 1.434762 \pi}, 0.312240 e^{i 0.694595 \pi}\right) \\
3,\left(0.670899 e^{i 1.289629 \pi}, 0.423652 e^{i 0.802981 \pi}\right)
\end{array}\right)
$$


Table 46 Tabulated form of $\mathrm{CFFNS}_{f} \mathrm{DM} \mathfrak{5}^{(4)}$ of expert $\mathfrak{D}_{4}$

\begin{tabular}{|c|c|c|c|}
\hline$\left(\mathcal{H}^{(4)}, \mathcal{Q}, 5\right)$ & $\mathfrak{B}_{1}$ & $\mathfrak{B}_{2}$ & $\mathfrak{B}_{3}$ \\
\hline $\begin{array}{l}\mathfrak{I}_{1} \\
\mathfrak{I}_{2} \\
\mathfrak{I}_{3} \\
\mathfrak{I}_{4}\end{array}$ & $\begin{array}{l}\left\langle 2,\left(0.45 e^{i 1.14 \pi}, 0.56 e^{i 0.91 \pi}\right)\right\rangle \\
\left\langle 3,\left(0.79 e^{i 1.65 \pi}, 0.29 e^{i 0.43 \pi}\right)\right\rangle \\
\left\langle 4,\left(0.88 e^{i 1.78 \pi}, 0.11 e^{i 0.36 \pi}\right)\right\rangle \\
\left\langle 2,\left(0.57 e^{i 1.25 \pi}, 0.48 e^{i 0.83 \pi}\right)\right\rangle\end{array}$ & $\begin{array}{l}\left\langle 2,\left(0.49 e^{i 0.92 \pi}, 0.50 e^{i 1.21 \pi}\right)\right\rangle \\
\left\langle 2,\left(0.47 e^{i 0.82 \pi}, 0.58 e^{i 1.08 \pi}\right)\right\rangle \\
\left\langle 3,\left(0.72 e^{i 1.58 \pi}, 0.31 e^{i 0.53 \pi}\right)\right\rangle \\
\left\langle 3,\left(0.67 e^{i 1.67 \pi}, 0.23 e^{i 0.69 \pi}\right)\right\rangle\end{array}$ & $\begin{array}{l}\left\langle 4,\left(0.94 e^{i 1.92 \pi}, 0.16 e^{i 0.31 \pi}\right)\right\rangle \\
\left\langle 2,\left(0.61 e^{i 1.20 \pi}, 0.59 e^{i 1.29 \pi}\right)\right\rangle \\
\left\langle 0,\left(0.08 e^{i 0.22 \pi}, 0.87 e^{i 1.79 \pi}\right)\right\rangle \\
\left\langle 2,\left(0.41 e^{i 1.18 \pi}, 0.64 e^{i 1.27 \pi}\right)\right\rangle\end{array}$ \\
\hline & $\mathfrak{B}_{4}$ & $\mathfrak{B}_{5}$ & $\mathfrak{B}_{6}$ \\
\hline $\begin{array}{l}\mathfrak{I}_{1} \\
\mathfrak{I}_{2} \\
\mathfrak{I}_{3} \\
\mathfrak{I}_{4}\end{array}$ & $\begin{array}{l}\left\langle 1,\left(0.22 e^{i 0.47 \pi}, 0.69 e^{i 1.43 \pi}\right)\right\rangle \\
\left\langle 4,\left(0.91 e^{i 1.89 \pi}, 0.05 e^{i 0.08 \pi}\right)\right\rangle \\
\left\langle 3,\left(0.81 e^{i 1.34 \pi}, 0.21 e^{i 0.57 \pi}\right)\right\rangle \\
\left\langle 4,\left(0.87 e^{i 1.72 \pi}, 0.13 e^{i 0.12 \pi}\right)\right\rangle\end{array}$ & $\begin{array}{l}\left\langle 4,\left(0.89 e^{i 1.72 \pi}, 0.17 e^{i 0.32 \pi}\right)\right\rangle \\
\left\langle 3,\left(0.76 e^{i 1.46 \pi}, 0.27 e^{i 0.43 \pi}\right)\right\rangle \\
\left\langle 0,\left(0.12 e^{i 0.25 \pi}, 0.91 e^{i 1.88 \pi}\right)\right\rangle \\
\left\langle 2,\left(0.51 e^{i 0.89 \pi}, 0.49 e^{i 1.02 \pi}\right)\right\rangle\end{array}$ & $\begin{array}{l}\left\langle 3,\left(0.73 e^{i 1.35 \pi}, 0.31 e^{i 0.59 \pi}\right)\right\rangle \\
\left\langle 1,\left(0.31 e^{i 0.63 \pi}, 0.77 e^{i 1.61 \pi}\right)\right\rangle \\
\left\langle 2,\left(0.52 e^{i 0.87 \pi}, 0.48 e^{i 0.98 \pi}\right)\right\rangle \\
\left\langle 2,\left(0.47 e^{i 0.98 \pi}, 0.57 e^{i 1.12 \pi}\right)\right\rangle\end{array}$ \\
\hline \multicolumn{4}{|c|}{$\mathfrak{B}_{7}$} \\
\hline $\begin{array}{l}\mathfrak{I}_{1} \\
\mathfrak{I}_{2} \\
\mathfrak{I}_{3} \\
\mathfrak{I}_{4}\end{array}$ & $\begin{array}{l}\left\langle 4,\left(0.91 e^{i 1.81 \pi}, 0.01 e^{i 0.24 \pi}\right)\right\rangle \\
\left\langle 2,\left(0.44 e^{i 0.81 \pi}, 0.59 e^{i 0.92 \pi}\right)\right\rangle \\
\left\langle 1,\left(0.21 e^{i 0.59 \pi}, 0.69 e^{i 1.34 \pi}\right)\right\rangle \\
\left\langle 4,\left(0.87 e^{i 1.72 \pi}, 0.12 e^{i 0.21 \pi}\right)\right\rangle\end{array}$ & & \\
\hline
\end{tabular}

Table 47 Tabulated form of $\mathrm{ACFFNS}_{f} \mathrm{DM}(\mathfrak{5}$

\begin{tabular}{|c|c|c|}
\hline$(\mathcal{H}, \mathcal{Q}, 5)$ & $\mathfrak{B}_{1}$ & $\mathfrak{B}_{2}$ \\
\hline $\begin{array}{l}\mathfrak{I}_{1} \\
\mathfrak{I}_{2} \\
\mathfrak{I}_{3} \\
\mathfrak{I}_{4}\end{array}$ & $\begin{array}{c}\left\langle 2,\left(0.384320 e^{i 0.89467 \pi}, 0.636677 e^{i 1.215568 \pi}\right)\right\rangle \\
\left\langle 3,\left(0.693071 e^{i 1.510336 \pi}, 0.334196 e^{i 0.615372 \pi}\right)\right\rangle \\
\left\langle 4,\left(0.921191 e^{i 1.84144 \pi}, 0.100947 e^{i 0.223135 \pi}\right)\right\rangle \\
\left\langle 2,\left(0.535991 e^{i 1.122995 \pi}, 0.444246 e^{i 0.959586 \pi}\right)\right\rangle\end{array}$ & $\begin{array}{c}\left.\left\langle 2,\left(0.525656 e^{i 1.098516 \pi}, 0.474341 e^{i 1.114966 \pi}\right)\right\rangle\right\rangle \\
\left\langle 2,\left(0.463232 e^{i 0.793682 \pi}, 0.613584 e^{i 1.247357 \pi}\right)\right\rangle \\
\left\langle 3,\left(0.57605 e^{i 1.341568 \pi}, 0.472935 e^{i 0.884443 \pi}\right)\right\rangle \\
\left\langle 3,\left(0.565281 e^{i 1.196937 \pi}, 0.448675 e^{i 1.085823 \pi}\right)\right\rangle\end{array}$ \\
\hline $\mathfrak{I}_{4}$ & $\mathfrak{B}_{3}$ & $\mathfrak{B}_{4}$ \\
\hline $\begin{array}{l}\mathfrak{I}_{1} \\
\mathfrak{I}_{2} \\
\mathfrak{I}_{3} \\
\mathfrak{I}_{4}\end{array}$ & $\begin{array}{l}\left\langle 4,\left(0.821814 e^{i 1.669277 \pi}, 0.297877 e^{i 0.539368 \pi}\right)\right\rangle \\
\left\langle 2,\left(0.626753 e^{i 1.176826 \pi}, 0.573407 e^{i 0.942689 \pi}\right)\right\rangle \\
\left\langle 1,\left(0.235591 e^{i 0.495882 \pi}, 0.800397 e^{i 1.553293 \pi}\right)\right\rangle \\
\left\langle 2,\left(0.426781 e^{i 1.156826 \pi}, 0.623415 e^{i 0.922475 \pi}\right)\right\rangle\end{array}$ & $\begin{array}{c}\left.\left\langle 2,\left(0.474829 e^{i 0.990753 \pi}, 0.609707 e^{i 1.307223 \pi}\right)\right\rangle\right\rangle \\
\left\langle 4,\left(0.805816 e^{i 1.591906 \pi}, 0.24108 e^{i 0.329189 \pi}\right)\right\rangle \\
\left\langle 4,\left(0.856807 e^{i 1.705982 \pi}, 0.101565 e^{i 0.312849 \pi}\right)\right\rangle \\
\left\langle 4,\left(0.740156 e^{i 1.535327 \pi}, 0.246763 e^{i 0.394609 \pi}\right)\right\rangle\end{array}$ \\
\hline & $\mathfrak{B}_{5}$ & $\mathfrak{B}_{6}$ \\
\hline $\begin{array}{l}\mathfrak{I}_{1} \\
\mathfrak{I}_{2} \\
\mathfrak{I}_{3} \\
\mathfrak{I}_{4}\end{array}$ & $\begin{array}{c}\left\langle 4,\left(0.925437 e^{i 1.773344 \pi}, 0.073543 e^{i 0.24464 \pi}\right)\right\rangle \\
\left\langle 3,\left(0.683699 e^{i 1.297906 \pi}, 0.399011 e^{i 0.765837 \pi}\right)\right\rangle \\
\left\langle 0,\left(0.10998 e^{i 0.236042 \pi}, 0.89715 e^{i 1.838062 \pi}\right)\right\rangle \\
\left\langle 3,\left(0.757506 e^{i 1.4106 \pi}, 0.320004 e^{i 0.596584 \pi}\right)\right\rangle\end{array}$ & $\begin{array}{c}\left\langle 4,\left(0.820664 e^{i 1.551716 \pi}, 0.157531 e^{i 0.548194 \pi}\right)\right\rangle \\
\left\langle 2,\left(0.48671 e^{i 0.874574 \pi}, 0.619182 e^{i 1.211136 \pi}\right)\right\rangle \\
\left\langle 2,\left(0.414822 e^{i 0.813233 \pi}, 0.644054 e^{i 1.336384 \pi}\right)\right\rangle \\
\left\langle 4,\left(0.56322 e^{i 1.110101 \pi}, 0.455531 e^{i 0.98608 \pi}\right)\right\rangle\end{array}$ \\
\hline \multicolumn{3}{|c|}{$\mathfrak{B}_{7}$} \\
\hline $\begin{array}{l}\mathfrak{I}_{1} \\
\mathfrak{I}_{2} \\
\mathfrak{I}_{3} \\
\mathfrak{I}_{4}\end{array}$ & $\begin{array}{l}\left\langle 4,\left(0.91033 e^{i 1.921529 \pi}, 0.064167 e^{i 0.293766 \pi}\right)\right\rangle \\
\left\langle 3,\left(0.586502 e^{i 1.096052 \pi}, 0.469085 e^{i 0.849702 \pi}\right)\right\rangle \\
\left\langle 1,\left(0.193176 e^{i 0.414263 \pi}, 0.824363 e^{i 1.688289 \pi}\right)\right\rangle \\
\left\langle 4,\left(0.814511 e^{i 1.658152 \pi}, 0.213975 e^{i 0.376418 \pi}\right)\right\rangle\end{array}$ & \\
\hline
\end{tabular}

- Step 4. The entries of AWCFFNS $\mathrm{AMM}_{f} \widehat{\mathrm{G}}$ are obtained by Equation 4 by utilizing $\mathrm{ACFFNS}_{f} \mathrm{DM}$, given by Table 47, and the weight vector $\kappa$ of attributes in Equation 10. These entries are tabulated, as shown in Table 49.

- Step 5. In the proposed MAGDM problem, the attributes topography, soil and water quality \& availability are benefit-type attributes whereas initial cost, environmental destruction, climate of the area and maintenance cost are cost-type attributes. $\mathrm{CFFNS}_{f}$-PIS and $\mathrm{CFFNS}_{f}$-NIS relative to each attribute, opted by Equations 5 and 6 , are arranged in Table 50.
- Step 6. Distance of each alternative from $\mathrm{CFFNS}_{f}$-PIS and $\mathrm{CFFNS}_{f}$-NIS is calculated by employing Equations 7 and 8 , respectively. These distance measures are tabulated in Table 51.

- Step 7. Table 52 represents the revised closeness index corresponding to each alternative, evaluated by using Equation 9.

- Step 8. The ranking of cities on the basis of revised closeness index is shown by Table 53. Since $\mathfrak{I}_{1}$ has maximum index value. Hence the experts will give suggestions to the investor to select Boston, Massachusetts for farming. 
Table 48 Importance weights of each attributes

\begin{tabular}{ccc}
\hline$(\mathcal{H}, \mathcal{Q}, 5)$ & $\mathfrak{D}_{1}$ & $\mathfrak{D}_{2}$ \\
\hline $\mathfrak{B}_{1}$ & $\left\langle 3,\left(0.67 e^{i 1.38 \pi}, 0.23 e^{i 0.43 \pi}\right)\right\rangle$ & $\left\langle 3,\left(0.74 e^{i 1.65 \pi}, 0.37 e^{i 0.73 \pi}\right)\right\rangle$ \\
$\mathfrak{B}_{2}$ & $\left\langle 4,\left(0.96 e^{i 1.79 \pi}, 0.09 e^{i 0.17 \pi}\right)\right\rangle$ & $\left\langle 4,\left(0.97 e^{i 1.92 \pi}, 0.07 e^{i 0.29 \pi}\right)\right\rangle$ \\
$\mathfrak{B}_{3}$ & $\left\langle 3,\left(0.71 e^{i 1.49 \pi}, 0.26 e^{i 0.52 \pi}\right)\right\rangle$ & $\left\langle 3,\left(0.82 e^{i 1.53 \pi}, 0.24 e^{i 0.49 \pi}\right)\right\rangle$ \\
$\mathfrak{B}_{4}$ & $\left\langle 1,\left(0.31 e^{i 0.49 \pi}, 0.67 e^{i 1.62 \pi}\right)\right\rangle$ & $\left\langle 0,\left(0.02 e^{i 0.21 \pi}, 0.89 e^{i 1.82 \pi}\right)\right\rangle$ \\
$\mathfrak{B}_{5}$ & $\left\langle 3,\left(0.68 e^{i 1.57 \pi}, 0.31 e^{i 0.67 \pi}\right)\right\rangle$ & $\left\langle 3,\left(0.69 e^{i 1.46 \pi}, 0.33 e^{i 0.76 \pi}\right)\right\rangle$ \\
$\mathfrak{B}_{6}$ & $\left\langle 3,\left(0.75 e^{i 1.63 \pi}, 0.27 e^{i 0.71 \pi}\right)\right\rangle$ & $\left\langle 3,\left(0.73 e^{i 1.33 \pi}, 0.25 e^{i 0.55 \pi}\right)\right\rangle$ \\
$\mathfrak{B}_{7}$ & $\left\langle 3,\left(0.81 e^{i 1.35 \pi}, 0.35 e^{i 0.47 \pi}\right)\right\rangle$ & $\left\langle 2,\left(0.43 e^{i 1.21 \pi}, 0.52 e^{i 0.92 \pi}\right)\right\rangle$ \\
\hline \hline & $\mathfrak{D}_{3}$ & $\mathfrak{D} 4$ \\
\hline $\mathfrak{B}_{1}$ & $\left\langle 3,\left(0.72 e^{i 1.37 \pi}, 0.28 e^{i 0.57 \pi}\right)\right\rangle$ & $\left\langle 3,\left(0.79 e^{i 1.56 \pi}, 0.34 e^{i 0.58 \pi}\right)\right\rangle$ \\
$\mathfrak{B}_{2}$ & $\left\langle 4,\left(0.89 e^{i 1.85 \pi}, 0.05 e^{i 0.22 \pi}\right)\right\rangle$ & $\left\langle 4,\left(0.88 e^{i 1.78 \pi}, 0.02 e^{i 0.27 \pi}\right)\right\rangle$ \\
$\mathfrak{B}_{3}$ & $\left\langle 4,\left(0.93 e^{i 1.73 \pi}, 0.12 e^{i 0.29 \pi}\right)\right\rangle$ & $\left\langle 4,\left(0.91 e^{i 1.82 \pi}, 0.14 e^{i 0.36 \pi}\right)\right\rangle$ \\
$\mathfrak{B}_{4}$ & $\left\langle 3,\left(0.76 e^{i 1.47 \pi}, 0.32 e^{i 0.45 \pi}\right)\right\rangle$ & $\left\langle 2,\left(0.57 e^{i 0.97 \pi}, 0.61 e^{i 1.17 \pi}\right)\right\rangle$ \\
$\mathfrak{B}_{5}$ & $\left\langle 4,\left(0.94 e^{i 1.75 \pi}, 0.08 e^{i 0.14 \pi}\right)\right\rangle$ & $\left\langle 4,\left(0.87 e^{i 1.79 \pi}, 0.09 e^{i 0.23 \pi}\right)\right\rangle$ \\
$\mathfrak{B}_{6}$ & $\left\langle 2,\left(0.62 e^{i 0.86 \pi}, 0.54 e^{i 1.02 \pi}\right)\right\rangle$ & $\left\langle 3,\left(0.83 e^{i 1.61 \pi}, 0.29 e^{i 0.65 \pi}\right)\right\rangle$ \\
$\mathfrak{B}_{7}$ & $\left\langle 2,\left(0.51 e^{i 0.92 \pi}, 0.43 e^{i 1.23 \pi}\right)\right\rangle$ & $\left\langle 3,\left(0.78 e^{i 1.55 \pi}, 0.36 e^{i 0.73 \pi}\right)\right\rangle$ \\
\hline
\end{tabular}

\section{Comparative analysis of CFFNS $_{f}$-TOPSIS technique}

In this section, we solve the MAGDM problem "Selection of the most suitable city in the USA for farming" by Fermatean fuzzy TOPSIS (FF-TOPSIS) method, proposed by Senapati and Yager (2020), to authenticate the importance and validity of proposed model. The step wise solution of MAGDM problem following the Fermatean fuzzy TOPSIS method is given as follows:

- Step 1. The linguistic terms along with grades are same as given in Table 42. Since the existing technique only deals with multi-attribute decision-making (MADM) problems. Hence, the aggregated opinion of all experts, given in Table 47 is used by the investor but the grading part is excluded and CFFNs have taken to be zero to apply FF-TOPSIS method. Fermatean fuzzy decision matrix (FFDM) is arranged in Table 54. Moreover, to determine the role of each criteria, the decision-maker sets the weights of attributes as follows:

$$
\lambda=(0.150 .20 .170 .10 .160 .130 .09)^{T}
$$

Step 2. The score of all FF numbers (FFNs) are determined to identify the Fermatean fuzzy positive and negative ideal solutions. The score of a FFN can be calculated by the following formula (Senapati and Yager 2020):

$$
S_{c}\left(\mathfrak{b}_{a_{g t}}\right)=s_{a_{g t}}^{3}-k_{a_{g t}}^{3} .
$$

The score values of all entries of FFDM are assembled in Table 55. Table 56 represents the FF-PIS and FF-NIS relative to each attribute.

- Step 3. Distance of each alternative $\mathfrak{I}_{g}$ from FF-PIS $\mathfrak{B}^{+}$ and FF-NIS $\mathfrak{B}^{-}$is computed by employing the equations as follows (Senapati and Yager 2020): $d\left(\mathfrak{I}_{g}, \mathfrak{G}^{+}\right)=$

$$
\frac{1}{2} \sum_{t=1}^{m} \lambda_{t} \sqrt{\frac{1}{2}\left\{\left(\mathbf{s}_{a_{g t}}^{3}-\left(s_{a_{t}}^{+}\right)^{3}\right)^{2}+\left(\mathbf{k}_{a_{g t}}^{3}-\left(k_{a_{t}}^{+}\right)^{3}\right)^{2}+\left(\left(\pi_{a_{g t}}^{3}-\left(\pi_{a_{t}}^{+}\right)^{3}\right)^{2}\right)\right\}}
$$
$d\left(\mathfrak{I}_{g}, \mathfrak{5}^{-}\right)=$

$$
\frac{1}{2} \sum_{t=1}^{m} \lambda_{t} \sqrt{\frac{1}{2}\left\{\left(\mathbf{s}_{a_{g_{t}}}^{3}-\left(s_{a_{t}}^{-}\right)^{3}\right)^{2}+\left(\mathbf{k}_{a_{g_{t}}}^{3}-\left(k_{a_{t}}^{-}\right)^{3}\right)^{2}+\left(\left(\pi_{a_{g_{t}}}^{3}-\left(\pi_{a_{t}}^{-}\right)^{3}\right)^{2}\right)\right\}}
$$

The results are tabulated in Table 57.

- Step 4. To find out the most suitable alternative, Table 58 represents the closeness index corresponding to each alternative which is evaluated by utilizing the Equation 9.

- Step 5. The increasing order ranking of cities is shown by Table 59, where 1 is for minimum closeness index value and 4 is for highest index value. Since $\mathfrak{I}_{1}$ has the maximum index value. Hence the investor will select Boston, Massachusetts for farming.

\subsection{Results}

1. Now, we present a comparison of the proposed technique with the existing FF-TOPSIS method (Senapati and Yager 2020) to assess the accuracy of $\mathrm{CFFNS}_{f}$ - 
Table 49 Tabular form of $\mathrm{AWCFFNS}_{f} \mathrm{DM} \widehat{\mathfrak{G}}$

Table $50 \mathrm{CFFNS}_{f}$-PIS and $\mathrm{CFFNS}_{f}$-NIS

\begin{tabular}{|c|c|c|}
\hline$(\mathcal{H}, \mathcal{Q}, 5)$ & $\mathfrak{B}_{1}$ & $\mathfrak{B}_{2}$ \\
\hline \multirow[t]{2}{*}{$\begin{array}{l}\mathfrak{I}_{1} \\
\mathfrak{I}_{2} \\
\mathfrak{I}_{3} \\
\mathfrak{I}_{4}\end{array}$} & $\begin{array}{l}\left\langle 2,\left(0.281249 e^{i 0.683147 \pi}, 0.653525 e^{i 1.249243 \pi}\right)\right\rangle \\
\left\langle 3,\left(0.507197 e^{i 1.153254 \pi}, 0.401287 e^{i 0.751931 \pi}\right)\right\rangle \\
\left\langle 3,\left(0.674138 e^{i 1.406076 \pi}, 0.308470 e^{0.593173 \pi}\right)\right\rangle \\
\left\langle 2,\left(0.392244 e^{i 0.857490 \pi}, 0.484229 e^{i 1.019488 \pi}\right)\right\rangle\end{array}$ & $\begin{array}{l}\left\langle 2,\left(0.496783 e^{i 1.022269 \pi}, 0.474560 e^{i 1.117885 \pi}\right)\right\rangle \\
\left\langle 2,\left(0.437787 e^{i 0.738593 \pi}, 0.613697 e^{i 1.249496 \pi}\right)\right\rangle \\
\left\langle 3,\left(0.544408 e^{i 1.248451 \pi}, 0.473156 e^{i 0.889553 \pi}\right)\right\rangle \\
\left\langle 3,\left(0.534231 e^{i 1.113859 \pi}, 0.448924 e^{i 1.088949 \pi}\right)\right\rangle\end{array}$ \\
\hline & $\mathfrak{B}_{3}$ & $\mathfrak{B}_{4}$ \\
\hline \multirow[t]{2}{*}{$\begin{array}{l}\mathfrak{I}_{1} \\
\mathfrak{I}_{2} \\
\mathfrak{I}_{3} \\
\mathfrak{I}_{4} \\
\end{array}$} & $\begin{array}{c}\left\langle 4,\left(0.707227 e^{i 1.378285 \pi}, 0.320871 e^{i 0.610807 \pi}\right\rangle\right. \\
\left\langle 2,\left(0.539364 e^{i 0.971679 \pi}, 0.578935 e^{i 0.966398 \pi}\right)\right\rangle \\
\left\langle 1,\left(0.202742 e^{i 0.409439 \pi}, 0.802114 e^{i 1.558591 \pi}\right)\right\rangle \\
\left\langle 2,\left(0.367274 e^{i 0.955166 \pi}, 0.627793 e^{i 0.947371 \pi}\right)\right\rangle\end{array}$ & $\begin{array}{l}\left\langle 2,\left(0.258992 e^{i 0.505084 \pi}, 0.740208 e^{i 1.508697 \pi}\right)\right\rangle \\
\left\langle 3,\left(0.439527 e^{i 0.811552 \pi}, 0.623269 e^{i 1.191945 \pi}\right)\right\rangle \\
\left\langle 3,\left(0.467339 e^{i 0.869707 \pi}, 0.614598 e^{i 1.191005 \pi}\right)\right\rangle \\
\left\langle 3,\left(0.403713 e^{i 0.782708 \pi}, 0.623937 e^{i 1.196714 \pi}\right)\right\rangle\end{array}$ \\
\hline & $\mathfrak{B}_{5}$ & $\mathfrak{B}_{6}$ \\
\hline $\begin{array}{l}\mathfrak{I}_{1} \\
\mathfrak{I}_{2} \\
\mathfrak{I}_{3} \\
\mathfrak{I}_{4}\end{array}$ & $\begin{array}{c}\left\langle 4\left(0.764928 e^{i 1.458701 \pi}, 0.189473 e^{i 0.430803 \pi}\right)\right\rangle \\
\left\langle 3,\left(0.565117 e^{i 1.067620 \pi}, 0.411197 e^{i 0.799440 \pi}\right)\right\rangle \\
\left\langle 0,\left(0.090905 e^{i 0.194161 \pi}, 0.897887 e^{i 1.839505 \pi}\right)\right\rangle \\
\left\langle 3,\left(0.626123 e^{i 1.160318 \pi}, 0.339023 e^{i 0.651104 \pi}\right)\right\rangle\end{array}$ & $\begin{array}{l}\left.\left\langle 3,0.607190 e^{i 1.113172 \pi}, 0.324696 e^{i 0.789955 \pi}\right)\right\rangle \\
\left\langle 2,\left(0.360106 e^{i 0.627403 \pi}, 0.638742 e^{i 1.267695 \pi}\right)\right\rangle \\
\left\langle 2,\left(0.306917 e^{i 0.583398 \pi}, 0.661504 e^{i 1.378904 \pi}\right)\right\rangle \\
\left\langle 3,\left(0.416714 e^{i 0.796365 \pi}, 0.496091 e^{i 1.078299 \pi}\right\rangle\right.\end{array}$ \\
\hline & $\mathfrak{B}_{7}$ & \\
\hline $\begin{array}{l}\mathfrak{I}_{1} \\
\mathfrak{I}_{2} \\
\mathfrak{I}_{3} \\
\mathfrak{I}_{4}\end{array}$ & $\begin{array}{l}\left\langle 3,\left(0.610740 e^{i 1.239030 \pi}, 0.424105 e^{i 0.815057 \pi}\right)\right\rangle \\
\left\langle 3,\left(0.393484 e^{i 0.706751 \pi}, 0.555490 e^{i 1.029621 \pi}\right)\right\rangle \\
\left\langle 1,\left(0.129602 e^{i 0.267123 \pi}, 0.840450 e^{i 1.712080 \pi}\right)\right\rangle \\
\left\langle 3,\left(0.546455 e^{i 1.069201 \pi}, 0.439838 e^{i 0.827983 \pi}\right)\right\rangle\end{array}$ & \\
\hline
\end{tabular}

Table 51 Distance of each alternative from ideal solution

\begin{tabular}{lll}
\hline Alternatives & $d\left(\mathfrak{\Im}_{g}, \widehat{\mathfrak{G}}_{t}\right)$ & $d\left(\mathfrak{\Im}_{g}, \breve{\mathfrak{b}}_{t}\right)$ \\
\hline $\mathfrak{I}_{1}$ & 1.002077 & 2.187073 \\
$\mathfrak{I}_{2}$ & 1.955608 & 1.434256 \\
$\mathfrak{I}_{3}$ & 2.274187 & 0.950996 \\
$\mathfrak{I}_{4}$ & 1.851251 & 1.589221 \\
\hline
\end{tabular}

TOPSIS method. Despite the difference in revised closeness index calculated by both techniques, the final ranking of cities is the same. Thus, the same city is proclaimed as the most suitable one for farming in both methods. The results of the proposed and existing methods, including the final ranking and best alternative, are summarized in Table 60 as follows:
Table 52 Revised closeness index of each alternative

Table 53 Ranking of each alternative

\begin{tabular}{lllll}
\hline Alternatives & $\mathfrak{J}_{1}$ & $\mathfrak{J}_{2}$ & $\mathfrak{I}_{3}$ & $\mathfrak{J}_{4}$ \\
Ranking & 4 & 2 & 1 & 3 \\
\hline
\end{tabular}


Table 54 Tabular representation of FFDM $\overrightarrow{\mathfrak{G}}$

\begin{tabular}{ccccc}
\hline & $\mathfrak{B}_{1}$ & $\mathfrak{B}_{2}$ & $\mathfrak{B}_{3}$ & $\mathfrak{B}_{4}$ \\
\hline $\mathfrak{I}_{1}$ & $(0.384320,0.636677)$ & $(0.525656,0.474341)$ & $(0.821814,0.297877)$ & $(0.474829,0.609707)$ \\
$\mathfrak{I}_{2}$ & $(0.693071,0.334196)$ & $(0.463232,0.613584)$ & $(0.626753,0.573407)$ & $(0.805816,0.241080)$ \\
$\mathfrak{I}_{3}$ & $(0.921191,0.100947)$ & $(0.576050,0.472935)$ & $(0.235591,0.800397)$ & $(0.856807,0.101565)$ \\
$\mathfrak{I}_{4}$ & $(0.535991,0.444246)$ & $(0.565281,0.448675)$ & $(0.426781,0.623415)$ & $(0.740156,0.246763)$ \\
\hline \hline & $\mathfrak{B}_{5}$ & $\mathfrak{B}_{6}$ & $\mathfrak{B}_{7}$ & \\
\hline $\mathfrak{I}_{1}$ & $(0.925437,0.073543)$ & $(0.820664,0.157531)$ & $(0.910330,0.064167)$ & \\
$\mathfrak{I}_{2}$ & $(0.683699,0.399011)$ & $(0.486710,0.619182)$ & $(0.586502,0.469085)$ & \\
$\mathfrak{I}_{3}$ & $(0.109980,0.897150)$ & $(0.414822,0.644054)$ & $(0.193176,0.824363)$ & \\
$\mathfrak{I}_{4}$ & $(0.757506,0.320004)$ & $(0.563220,0.455531)$ & $(0.814511,0.213975)$ & \\
\hline & & & & \\
\hline
\end{tabular}

Table 55 Score values of FFNs to opt ideal solutions

\begin{tabular}{llllllll}
\hline & $\mathfrak{B}_{1}$ & $\mathfrak{B}_{2}$ & $\mathfrak{B}_{3}$ & $\mathfrak{B}_{4}$ & $\mathfrak{B}_{5}$ & $\mathfrak{B}_{6}$ & $\mathfrak{B}_{7}$ \\
\hline $\mathfrak{I}_{1}$ & -0.20132 & 0.038520 & 0.528605 & -0.11960 & 0.792178 & 0.548798 & 0.754126 \\
$\mathfrak{I}_{2}$ & 0.295590 & -0.131603 & 0.057666 & 0.509237 & 0.256065 & -0.12209 & 0.098530 \\
$\mathfrak{I}_{3}$ & 0.780688 & 0.085372 & -0.49969 & 0.627949 & -0.72077 & -0.19578 & -0.55301 \\
$\mathfrak{I}_{4}$ & 0.066309 & 0.090309 & -0.16455 & 0.390455 & 0.401899 & 0.084136 & 0.530573 \\
\hline
\end{tabular}

Table 56 FF-PIS and FF-NIS

\begin{tabular}{lll}
\hline Attributes & FF-PIS $\left(\mathfrak{G}^{+}\right)$ & $\mathrm{B}_{p} \mathrm{FNS}_{f}$-NIS $\left(\mathfrak{6}^{-}\right)$ \\
\hline $\mathfrak{B}_{1}$ & $(0.384320,0.636677)$ & $(0.921191,0.100947)$ \\
$\mathfrak{B}_{2}$ & $(0.463232,0.613584)$ & $(0.565281,0.448675)$ \\
$\mathfrak{B}_{3}$ & $(0.821814,0.297877)$ & $(0.235591,0.800397)$ \\
$\mathfrak{B}_{4}$ & $(0.474829,0.609707)$ & $(0.856807,0.101565)$ \\
$\mathfrak{B}_{5}$ & $(0.109980,0.897150)$ & $(0.925437,0.073543)$ \\
$\mathfrak{B}_{6}$ & $(0.820664,0.157531)$ & $(0.414822,0.644054)$ \\
$\mathfrak{B}_{7}$ & $(0.910330,0.064167)$ & $(0.193176,0.824363)$ \\
\hline
\end{tabular}

Table 57 Distance of each alternative from ideal solution

\begin{tabular}{lll}
\hline Alternatives & $d\left(\mathfrak{I}_{g}, \mathfrak{5}^{+}\right)$ & $d\left(\mathfrak{I}_{g}, \mathfrak{5}^{-}\right)$ \\
\hline $\mathfrak{I}_{1}$ & 0.071595 & 0.174813 \\
$\mathfrak{I}_{2}$ & 0.152928 & 0.130212 \\
$\mathfrak{I}_{3}$ & 0.182976 & 0.062972 \\
$\mathfrak{I}_{4}$ & 0.151493 & 0.137461 \\
\hline
\end{tabular}

2. A comparison chart is designed in Figure 3 to envision the conformity of final results of compared and proposed MAGDM approaches which shows the effectuality and accountability of our proposed technique.

3. It is clear from the figure that both techniques elucidate the same outcome and ranking order that indicates the feasibility and sustain-ability of the presented technique.
Table 58 Revised closeness index of each alternative

\begin{tabular}{ll}
\hline Alternatives & $\psi\left(\mathfrak{I}_{g}\right)$ \\
\hline $\mathfrak{I}_{1}$ & 0 \\
$\mathfrak{I}_{2}$ & -1.39116 \\
$\mathfrak{I}_{3}$ & -2.19548 \\
$\mathfrak{I}_{4}$ & -1.32965 \\
\hline
\end{tabular}

Table 59 Ranking of each alternative

\begin{tabular}{lllll}
\hline Alternatives & $\mathfrak{I}_{1}$ & $\mathfrak{I}_{2}$ & $\mathfrak{I}_{3}$ & $\mathfrak{I}_{4}$ \\
Ranking & 4 & 2 & 1 & 3 \\
\hline
\end{tabular}

4. Our proposed CFFNS $_{f}$-TOPSIS technique has capability to handle the vagueness and periodicity involve in the data simultaneously, but the compared FF-TOPSIS technique is limited to capture the ambiguity of nonperiodic data that may cause to the inconsistency and specious outcomes. This extraordinary trait of the proposed strategy depicts that it is the more effective and generalized MAGDM strategy.

5. Due to the inadequacy of multi-valued grades and periodic terms, FF-TOPSIS cannot deal with CFFNS $_{f}$ information. On the other hand, $\mathrm{CFFNS}_{f}$-TOPSIS method has potential to handle the FF information by taking phase terms equal to zero and neglecting the grades. Since the results are same in both cases which 
depicts the proposed method more adaptable than existing methods.

\section{Merits of $\mathrm{CFFNS}_{f} \mathrm{~S}$ model and $\mathrm{CFFNS}_{f}$ TOPSIS approach}

1. In the modern era, the performance appraisal system is commonly used for the rating of restaurant management, schools, candidates for job, online services, online applications, products and websites, etc. The proposed model is designed to handle the rating-based assessment framework along with imprecise and vague two-dimensional information.

2. In this article, the robust technique of CFFNS $_{f}$-TOPSIS is developed for determining the best solution obtaining the closest distance from PIS and far away from NIS. The framework of the presented MAGDM strategy has remarkable aspects: it merges the fascinating advantages of TOPSIS with the hybrid model of $\mathrm{CFFNS}_{f} \mathrm{~S}$. The advantage of the hybrid model is that it has the potency to handle vagueness and periodicity of parameterized graded information simultaneously.

3. The proposed model shows the same accuracy when applied to the existing models inclusive of FF, CIF, CPF, $\mathrm{FFS}_{f}, \mathrm{CIFS}_{f}, \mathrm{CPFS}_{f}, \mathrm{FFNS}_{f}, \mathrm{CIFNS}_{f}$, and $\mathrm{CPFNS}_{f}$ by taking either $N=2$ or substituting phase terms equal to zero or by applying both strategies. Hence, the developed technique deprives a adaptable tool that skillfully and efficiently accomplishes its decision-making chores with preciseness under traditional as well as two-dimensional vague information along with finely-graded parameters.

\section{Conclusion}

Decision-making methods play an important role in the real life of human beings. The process of choosing the best option among a set of possible options is present in all human activities. In this paper, a new theory has been developed that serves as a mathematical tool which deals

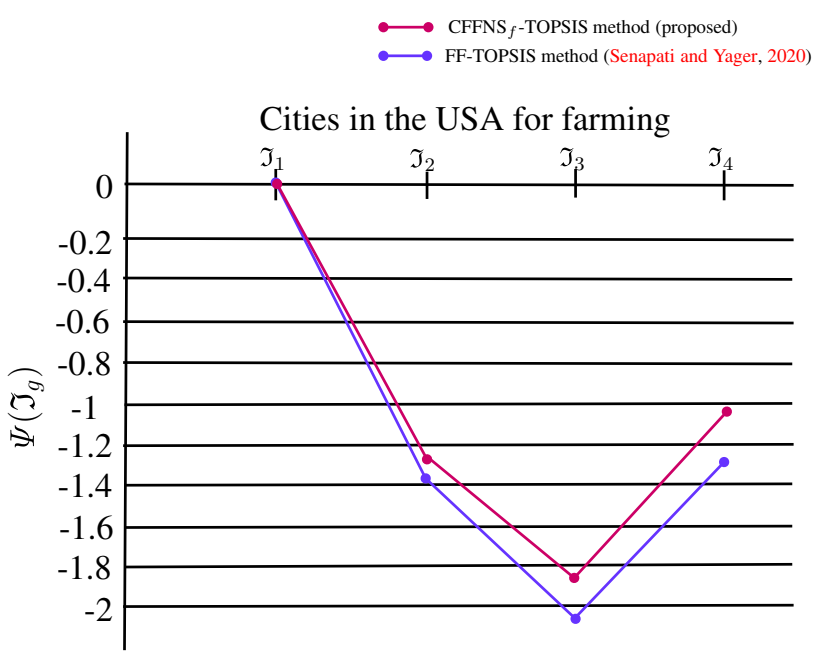

Fig. 3 Comparative analysis

with the two-dimensional vague information, and which is a generalization of the fuzzy $N$-soft set. We have advanced a model, $\mathrm{CFFNS}_{f} \mathrm{~S}$, that assesses the uncertain and vague data which has complex membership and non-membership values, parameterized information, and ordinal ranking systems. To establish a comparison between two $\mathrm{CFFNS}_{f} \mathrm{Ns}$, we have developed score and accuracy functions in a CFFNS $\mathrm{CH}_{f}$ environment. We have defined some basic operations for the $\mathrm{CFFNS}_{f} \mathrm{~S}$ model that include: complement (weak complement, $\mathrm{CFFNS}_{f} \mathrm{~S}$ complement, and weak $\mathrm{CFFNS}_{f} \mathrm{~S}$ complement), union (extended union and restricted union), intersection (extended intersection and restricted intersection). We have also included relevant examples for these operations. In addition, we have presented algebraic and Yager operations for $\mathrm{CFFNS}_{f} \mathrm{Ns}$.

Moreover, we have accomplished three algorithms to resolve multi-attribute decision-making problems. These algorithms have been validated by two real-life examples related to the selection of cars and the selection of the best telecommunication company in Pakistan.

Furthermore, in order to analyze the validity, feasibility, and reliability of the proposed model, we have conducted a comparative study of our approach with two operators: the $\mathrm{FFY}_{w} \mathrm{G}$ operator and the $\mathrm{FFY}_{w} \mathrm{~A}$ operator.

With respect to the proposed CFFNS $_{f}$-TOPSIS method, our method possesses the MAGDM potential of TOPSIS along with the adequacy of the proposed $\mathrm{CFFNS}_{f}$ model to

Table 60 Comparative analysis

\begin{tabular}{ll}
\hline Methods & Ranking of the most suitable city for farming \\
\hline CFFNS $_{f}$-TOPSIS method (proposed) & $\mathfrak{I}_{3} \prec \mathfrak{I}_{2} \prec \mathfrak{I}_{4} \prec \mathfrak{I}_{1}$ \\
FF-TOPSIS method (Senapati and Yager 2020) & $\mathfrak{I}_{3} \prec \mathfrak{I}_{2} \prec \mathfrak{I}_{4} \prec \mathfrak{I}_{1}$ \\
\hline
\end{tabular}


improve the exactness of decision-making results. The proposed method's primary dominance is due to its capability to tackle two-dimensional imprecise information along with level of attributes based on alternative with the help of $N$-soft grading values as well as complex membership and non-membership values. The basic principle of the $\mathrm{CFFNS}_{f}$-TOPSIS method is to find out the best solution possessing the proximity distance from the ideal solutions.

In the presented approach, the primary information has been equipped by ordered grades and their corresponding $\mathrm{CFFNS}_{f}$ Ns. The individual opinions have been aggregated by employing $\mathrm{CFFNS}_{f} \mathrm{WA}$ operator. Further, the $\mathrm{AWCFFNS}_{f} \mathrm{DM}$ has been acquired by the multiplication of $\mathrm{CFFNS}_{f}$ weight vector of criteria and $\mathrm{CFFNS}_{f}$ DM. After examining the $\mathrm{CFFNS}_{f}$-PIS and $\mathrm{CFFNS}_{f}$-NIS, distance of each alternative from ideal solutions have been computed. Further, the revised closeness index of each alternative has been calculated by evolving the discrepancy of these variables from the ideal solution. After the evaluated results of the closeness index, the alternatives are arranged in an ascending order. The alternative having maximum value of closeness index will be the optimal solution of the MAGDM problem. The proposed approach has been endorsed by a numerical example related to the selection of the suitable city in the USA for farming.

Along with beneficial characteristics of the proposed technique based on TOPSIS method for MAGDM problems in two-dimensional data, it ensures the same level of authenticity under Fermatean fuzzy environment by eliminating the grades and substituting phase terms equal to zero. On the contrary, the adeptness of the FF-TOPSIS method is restricted to handle one dimensional phenomena, also it is unable to deal with MAGDM problems.

Moreover, the proposed CFFNS $_{f}$-TOPSIS method has an edge over the extant decision-making approaches as the $\mathrm{CFFNS}_{f} \mathrm{~S}$ model can effectively apply in the environments of FFS, CIFS, CPFS, $\mathrm{FFS}_{f} \mathrm{~S}, \mathrm{FNS}_{f}, \mathrm{IFNS}_{f} \mathrm{~S}, \mathrm{PFNS}_{f} \mathrm{~S}$, $\mathrm{CPFNS}_{f}$ and so forth by taking either $N=2$ or substituting phase terms equal to zero or by applying both strategies.

Funding Open Access funding provided thanks to the CRUE-CSIC agreement with Springer Nature.

\section{Declarations}

Conflict of interest The authors declare that they have no conflict of interest.

Ethical approval This article does not contain any studies with human participants or animals performed by any of the authors.

Open Access This article is licensed under a Creative Commons Attribution 4.0 International License, which permits use, sharing, adaptation, distribution and reproduction in any medium or format, as long as you give appropriate credit to the original author(s) and the source, provide a link to the Creative Commons licence, and indicate if changes were made. The images or other third party material in this article are included in the article's Creative Commons licence, unless indicated otherwise in a credit line to the material. If material is not included in the article's Creative Commons licence and your intended use is not permitted by statutory regulation or exceeds the permitted use, you will need to obtain permission directly from the copyright holder. To view a copy of this licence, visit http://creativecommons. org/licenses/by/4.0/.

\section{References}

Abdullah ML, Abdullah WSW, Tap AOM (2012) Fuzzy sets in the social sciences: an overview of related researches. J Teknol 41(1):43-54

Akram M, Adeel A (2019) TOPSIS approach for MAGDM based on interval-valued hesitant fuzzy $N$-soft environment. Int J Fuzzy Syst 21(3):993-1009

Akram M, Naz S (2019) A novel decision-making approach under complex Pythagorean fuzzy environment. Math Comput Appl 24(3):73

Akram M, Adeel A, Alcantud JCR (2018) Fuzzy $N$-soft sets: A novel model with applications. J Intell Fuzzy Syst 35(4):4757-4771

Akram M, Adeel A, Alcantud JCR (2019a) Group decision making methods based on hesitant $N$-soft sets. Experts Syst Appl 115:95-105

Akram M, Ali G, Alcantud JCR (2019b) New decision-making hybrid model: intuitionistic fuzzy $N$-soft rough sets. Soft Comput 23(20):9853-9868

Akram M, Dudek WA, Ilyas F (2019c) Group decision-making based on Pythagorean fuzzy TOPSIS method. Int J Intell Syst 34(7):1455-1475

Akram M, Garg H, Zahid K (2020) Extensions of ELECTRE-I and TOPSIS methods for group decision-making under complex Pythagorean fuzzy environment. Iran J Fuzzy Syst 17(5):147-164

Akram M, Amjad U, Davvaz B (2021) Decision-making analysis based on bipolar fuzzy $N$-soft information. Comput Appl Math 40(6): 1-39

Akram M, Shabir M, Adeel A, Al-Kenani AN (2021) A multiattribute decision-making framework: VIKOR method with complex spherical fuzzy $N$-soft sets. Math Prob Eng 2021:1490807

Akram M, Shabir M, Al-Kenani AN, Alcantud JCR (2021) Hybrid decision-making frameworks under complex spherical fuzzy $N$ soft sets. J Math

Akram M, Wasim F, Al-Kenani AN (2021) Complex q-rung orthopair fuzzy $N$-soft sets: a new model with applications. Complexity 2021:3690597

Akram M, Wasim F, Al-Kenani AN (2021) A hybrid decision-making approach under complex Pythagorean fuzzy $N$-soft sets. Int $\mathbf{J}$ Comput Intell Syst 14(1):1263-1291

Alcantud JCR (2016) A novel algorithm for fuzzy soft set based decision making from multiobserver input parameter data set. Inf Fus 29:142-148

Alcantud JCR, Andrés Calle R (2017) The problem of collective identity in a fuzzy environment. Fuzzy Sets Syst 315:57-75

Alcantud JCR, Laruelle A (2014) Dis and approval voting: a characterization. Soc Choice Welf 43(1):1-10

Alcantud JCR, Santos-García G (2017) A new criterion for soft set based decision making problems under incomplete information. Int J Comput Intell Syst 10(1):394-404 
Alcantud JCR, Rambaud SC, Torrecillas MJM (2017) Valuation fuzzy soft sets: a flexible fuzzy soft set based decision making procedure for the valuation of assets. Symmetry 9(11):253

Alkouri AM, Salleh AR (2012) Complex intuitionistic fuzzy sets. In: AIP conference proceedings, American Institute of Physics 1482(1):464-470

Atanassov KT (1986) Intuitionistic fuzzy sets. Fuzzy Sets Syst 20:87-96

Boran FE, Genç S, Kurt MM, Akay D (2009) A multi-criteria intuitionistic fuzzy group decision making for supplier selection with TOPSIS method. Expert Syst Appl 36(8):11363-11368

Boran FE, Genç S, Akay D (2011) Personnel selection based on intuitionistic fuzzy sets. Hum Factors Ergon Manuf Serv Ind 21(5):493-503

Boran FE, Boran K, Menlik T (2012) The evaluation of renewable energy technologies for electricity generation in Turkey using intuitionistic fuzzy TOPSIS. Energy Sources Part B Econ Plan Policy 7(1):81-90

Chen CT (2000) Extension of the TOPSIS for group decision-making under fuzzy environment. Fuzzy Sets Syst 114(1):1-9

Chen S, Liu J, Wang H, Augusto JC (2013) Ordering based decisionmaking a survey. Inf Fus 14(4):521-531

Eraslan S (2015) A decision making method via TOPSIS on soft sets. J New Results Sci 4(8):57-71

Eraslan S, Karaaslan F (2015) A group decision making method based on TOPSIS under fuzzy soft environment. J New Theory 3:30-40

Fatimah F, Alcantud JCR (2021) The multi-fuzzy $N$-soft set and its applications to decision-making. Neural Comput Appl 33:11437-11446

Fatimah F, Rosadi D, Hakim RBF, Alcantud JCR (2018) $n$-soft sets and their decision making algorithms. Soft Comput 22(4):3829-3842

Fatimah F, Rosadi D, Hakim RBF, Alcantud JCR (2019) Probabilistic soft sets and dual probabilistic soft sets in decision-making. Neural Comput Appl 31(1):397-407

Garg H, Shahzadi G (2020) Akram M (2020) Decision-making analysis based on Fermatean fuzzy Yager aggregation operators with application in COVID-19 testing facility. Math Prob Eng 4:7279027

Guiffrida AL, Nagi R (1998) Fuzzy set theory applications in production management research: a literature survey. J Intell Manuf 99(1):39-56

Han Q, Li W, Song Y, Zhang T, Wang R (2019) A new method for MAGDM based on improved TOPSIS and a novel Pythagorean fuzzy soft entropy. Symmetry 11(7):905

Herawan T, Deris MM (2009) On multi-soft set construction in information systems. In: International Conference on Intelligent Computing. Springer, Berlin, pp 101-110
Hwang CL, Yoon K (1981) Multiple attributes decision making: methods and applications a state-of-the-art survey. Springer, Berlin

Li J, Xu X, Yao Z, Lu Y (2019) Improving service quality with the fuzzy TOPSIS method: a case study of the Beijing rail transit system. IEEE Access 7:114271-114284

Ma X, Liu Q, Zhang J (2017) A survey decision-making method based on certain hybrid soft set models. Artif Intell Rev 47(4):507-530

Maji PK, Biswas R, Roy AR (2001) Intuitionistic fuzzy soft sets. J Fuzzy Math 9(3):677-692

Maji PK, Roy AR, Biswas R (2001) Fuzzy soft sets. J Fuzzy Math 9(3):589-602

Molodtsov DA (1999) Soft set theory-first results. Comput Math Appl 37(4-5):19-31

Molodtsov DA (2004) The theory of soft sets. URSS Publishers, Moscow ((in Russian))

Peng X, Yang Y, Song J (2015) Pythagorean fuzzy soft set and its application. Comput Eng 41(7):224-229

Ramot D, Milo R, Friedman M, Kandel A (2002) Complex fuzzy sets. IEEE Trans Fuzzy Syst 10(2):171-186

Salsabeela V, John SJ (2021) TOPSIS techniques on Fermatean fuzzy soft sets. AIP Conf Proc 2336(1):40022

Senapati T, Yager RR (2020) Fermatean fuzzy sets. J Ambient Intell Humaniz Comput 11(2):663-674

Sivadas A, John SJ (2020) Fermatean fuzzy soft sets and its applications. In: International conference on computational sciences-modelling, computing and soft, pp 203-216

Ullah K, Mahmood T, Ali Z, Jan N (2020) On some distance measures of complex Pythagorean fuzzy sets and their applications in pattern recognition. Complex and Intelligent Systems 6(1):15-27

Vencheh AH, Mirjaberi M (2014) Fuzzy inferior ratio method for multiple attribute decision making problems. Inf Sci 277:263-272

Yager RR (2013a) Pythagorean fuzzy subsets. In: 2013 Joint IFSA world congress and NAFIPS annual meeting (IFSA/NAFIPS), IEEE, pp 57-61

Yager RR (2013) Pythagorean membership grades in multicriteria decision making. IEEE Trans Fuzzy Syst 22(4):958-965

Yager RR (2016) Generalized orthopair fuzzy sets. IEEE Trans Fuzzy Syst 25(5):1222-1230

Zadeh LA (1965) Fuzzy sets. Inf Control 8(3):338-353

Zhang H, Jia-hua D, Yan C (1965) Multi-attribute group decisionmaking methods based on Pythagorean fuzzy $N$-soft sets. IEEE Access 8:62298-62309

\section{Authors and Affiliations}

\section{Muhammad Akram ${ }^{1} \cdot$ Umaira Amjad $^{1} \cdot$ José Carlos R. Alcantud $^{2} \cdot$ Gustavo Santos-García $^{3}$}

Gustavo Santos-García

santos@usal.es

Muhammad Akram

m.akram@pucit.edu.pk

Umaira Amjad

umairaamjad1@gmail.com

José Carlos R. Alcantud

jcr@usal.es
1 Department of Mathematics, University of the Punjab, New Campus, Lahore, Pakistan

2 BORDA Research Unit and IME, University of Salamanca, 37007 Salamanca, Spain

3 IME, University of Salamanca, 37007 Salamanca, Spain 